\title{
Characterization of the Role of aeneas in Primordial Germ Cell Migration and Blastoderm Cellularization
}

\author{
Dissertation \\ Submitted to the \\ Georg-August-University Göttingen, Faculty of Biology, \\ for the Degree of Doctor rerum naturalium (Dr. rer. nat.)
}

by

Roland Graf

born in Hannover, 14.11.1977 
meiner Familie 


\section{Affidavit}

Here I declare that my thesis entitled "Characterization of the Role of aeneas in Primordial Germ Cell Migration and Blastoderm Cellularization" has been done independently and with no other sources and aids than quoted.

Roland Graf 


\section{Table of Contents}

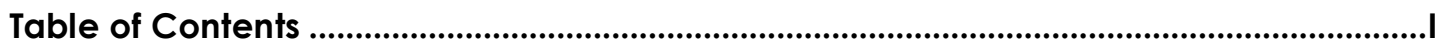

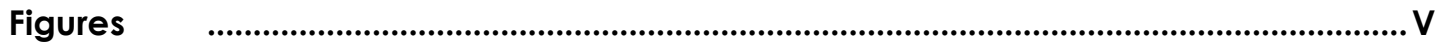

Tables

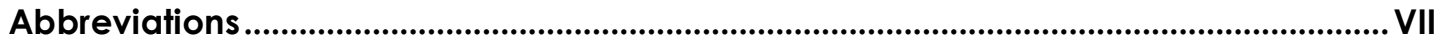

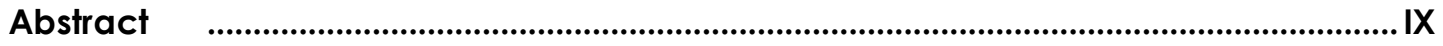

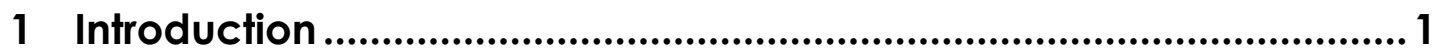

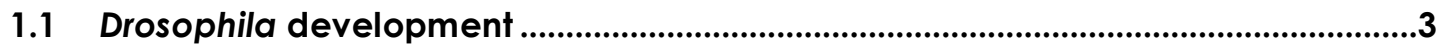

1.1.1 Oogenesis in Drosophila melanogaster .......................................................................

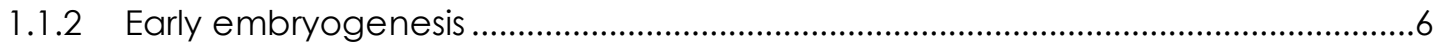

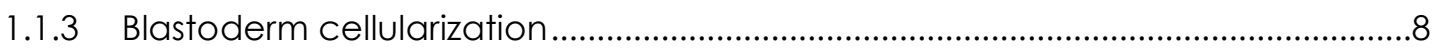

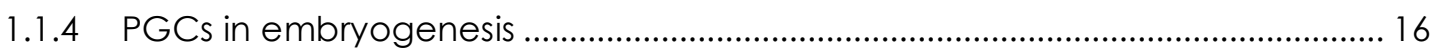

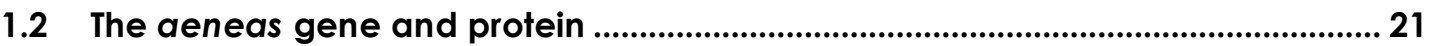

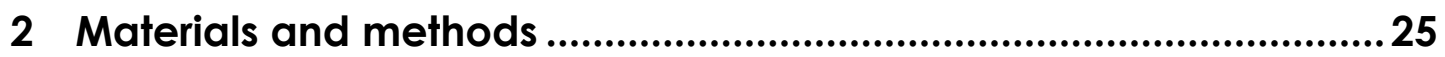



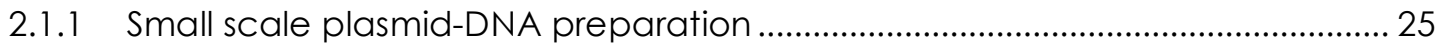

2.1.2 Medium scale plasmid DNA preparation............................................................. 25

2.1.3 Large scale plasmid DNA preparation .................................................................... 25

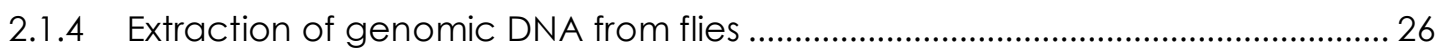

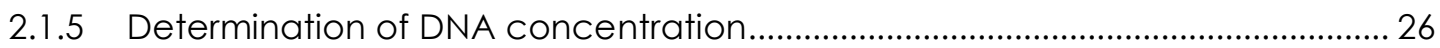

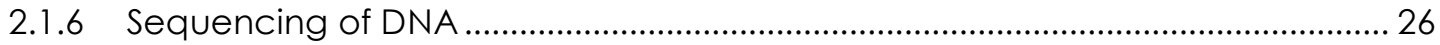

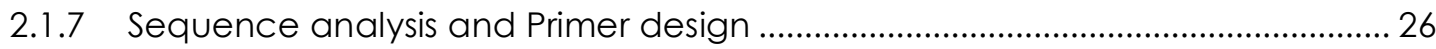

2.1.8 Sequence IDs of the sequences included in the phylogenetic tree of AenA. 27



2.1.10 DNA digest with restriction endonucleases............................................................. 28

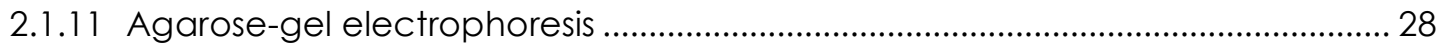

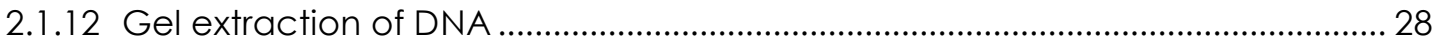

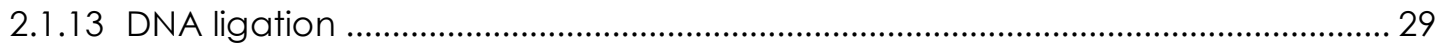


2.1.14 Gateway recombination 29

2.1.15 Heatshock transformation of bacterial strains ......................................................... 29

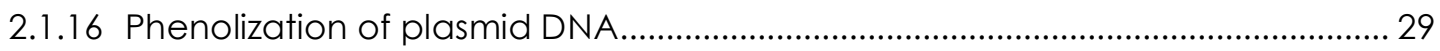

2.1.17 Preparation of injection mixture from Plasmid DNA …...................................... 30

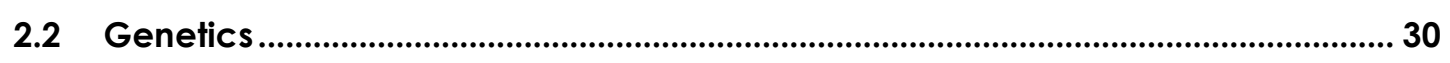

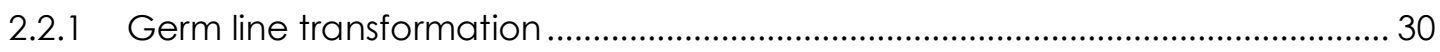

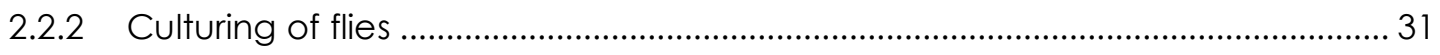

2.2.3 Crosses for the genetic interaction analysis between aen and slam ............... 31

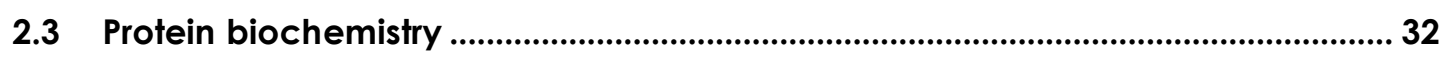

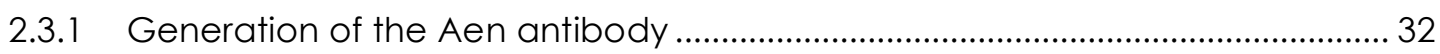

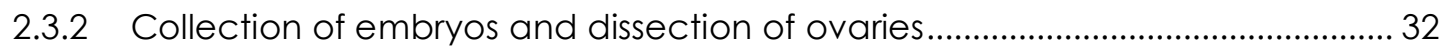

2.3.3 Protein extraction from embryos and ovaries ......................................................... 33

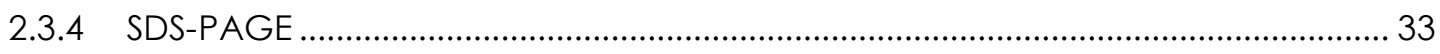

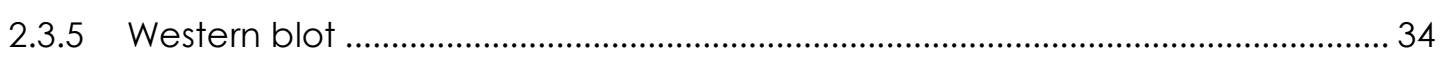

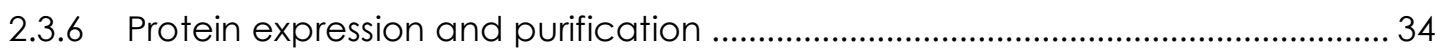

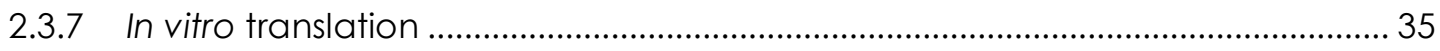

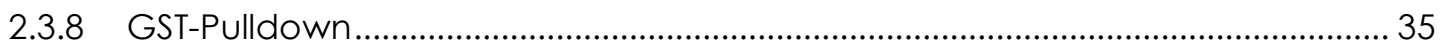

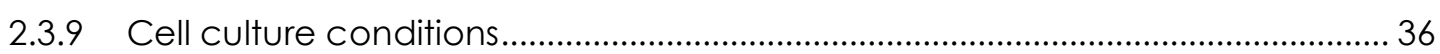

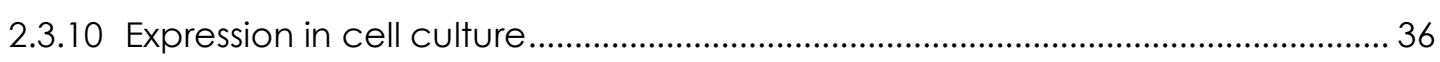

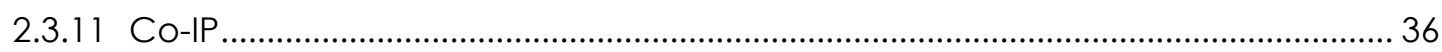

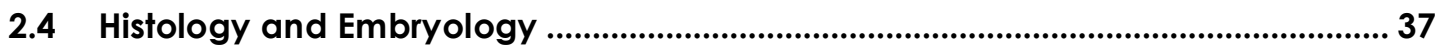

2.4.1 Formaldehyde fixation of Drosophila embryos ....................................................... 37

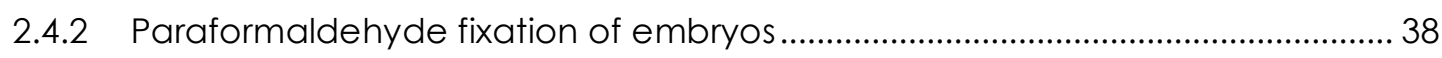

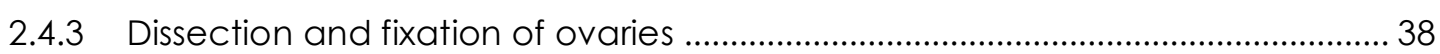

2.4.4 Generation of transcript specific probes for RNA in situ detection......................39

2.4.5 RNA in situ detection in fixated embryos and ovaries ...........................................39

2.4.6 Antibody staining of Drosophila embryos ............................................................... 40

2.4.7 Microscope / Confocal imaging .......................................................................... 41

2.4.8 In vivo imaging of embryonic development........................................................ 41

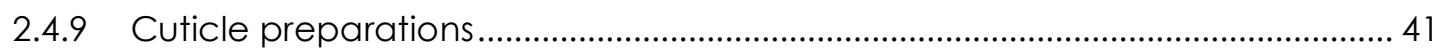

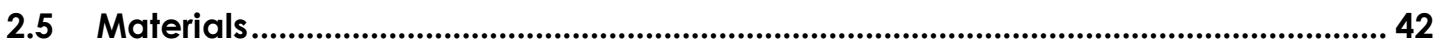

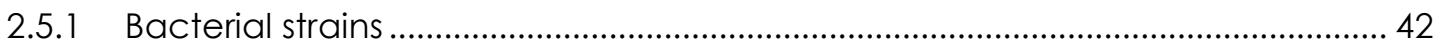

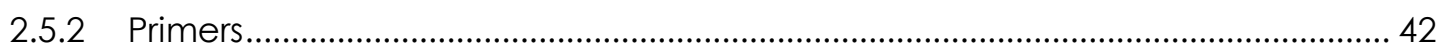

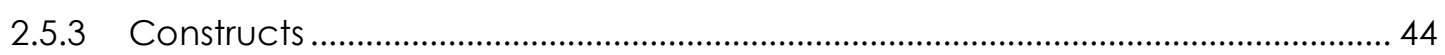

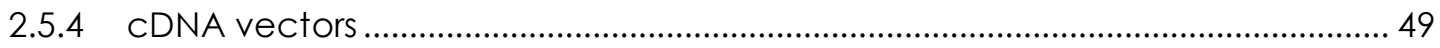

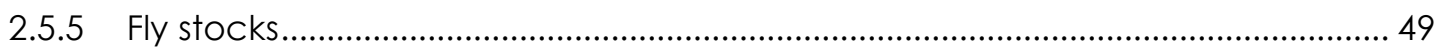

2.5.6 Antibodies used for Western blot detection and co-immunoprecipitation..... 50 
3.1 Conservation of the Aeneas proteins

3.2 Characterization of the phenotype observed in aen mutant embryos

3.2.1 aen mutant embryos show a strong increase in untimely PGC migration ....... 55

3.2.2 In vivo imaging of PGC migration in aen mutant embryos ................................. 57

3.2.3 The PGC transmigration phenotype in aen mutants can be rescued by introducing a genomic fragment covering the aen locus.

3.2.4 The PGC transmigration phenotype in aen mutants can be rescued by Gal4-

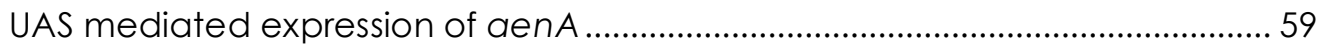

3.3 Expression of aen

3.3.1 aen transcripts are restricted to the germline during oogenesis

3.3.2 The Aen-antibody specifically detects both Aen protein variants on Western blots

3.3.3 AenA and AenB are differentially translated in the course of development .. 64

3.4 The subcellular localization of Aen.

3.4.1 The anti-Aen antibody specifically detects Aen in fixed tissue. 65

3.4.2 Aen shows a partial overlap with the recycling endosome marker Rab 11 ..... 68

3.4.3 Aen shows a partial overlap with the Golgi apparatus marker Lava lamp ..... 69

3.4.4 Aen partially co-localizes with the Microtubules ................................................... 71

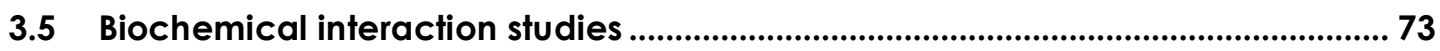

3.5.1 In vitro interaction studies of Aen with Arpl, Slam and Shg ............................. 74

3.5.2 AenA and AenB interact with Arpl and Slam in co-immunoprecipitation experiments 77

3.5.3 Mapping of the Aen interacting domains of Slam ................................................ 79

3.6 Co-localization of Aen with its interaction partners .............................................. 80

3.6.1 Aen co-localizes with Dynein Heavy Chain ............................................................. 80

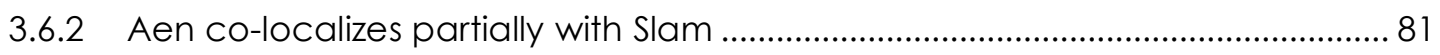

3.7 Genetic interaction studies of aen with slam ............................................................... 83

3.7.1 Genetic interaction of aen and slam during cellularization ............................... 83

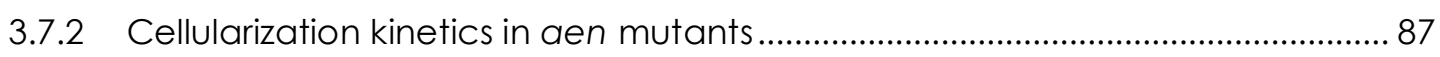

3.7.3 Cuticle phenotypes in single and double mutants of aen and slam ............... 88

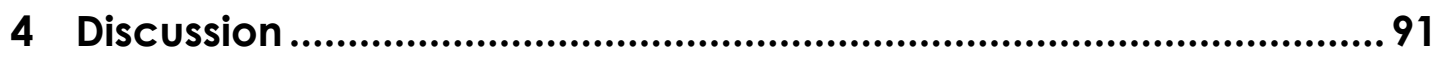

4.1 Correlation between aen expression, phenotype and localization ......................92 


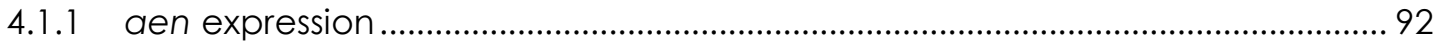

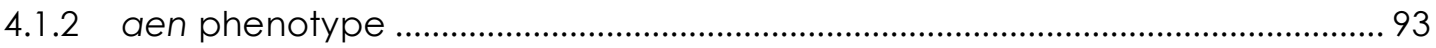

4.1.3 Subcellular localization of Aen in the somatic blastoderm ............................... 93

4.1.4 Functional correlation of aen with rabl 1 mutants ................................................ 94

4.1.5 Functional correlation of aen with the IVa LOF .................................................... 95

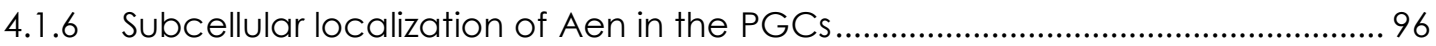

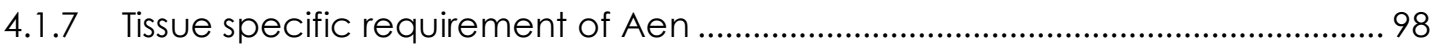

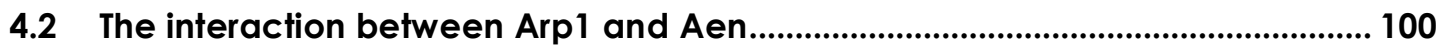

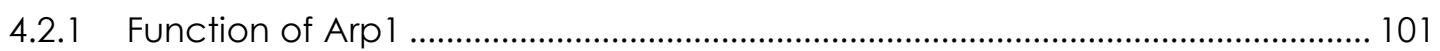

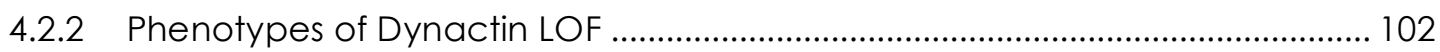

4.2.3 Functional correlation between Dynactin and Aen ......................................... 103

4.3 The interaction between Slam and Aen ..................................................................... 104

4.3.1 Functional models for Aen Slam interaction......................................................... 107

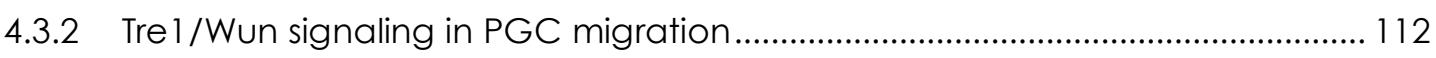

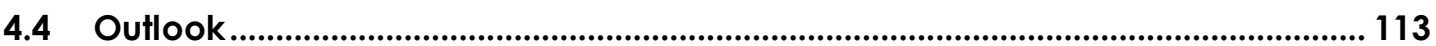

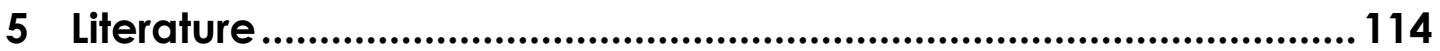

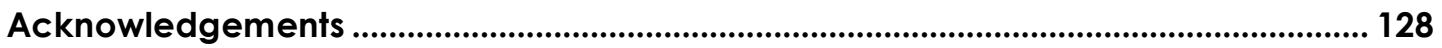

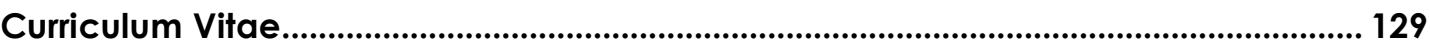

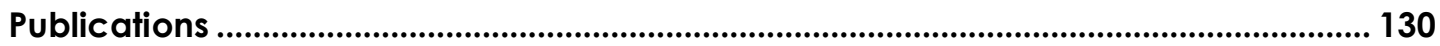




\section{Figures}



Figure 2: Syncytial stages of embryonic development ........................................................



Figure 4: Embryonic stages in respect to PGC development ........................................ 17

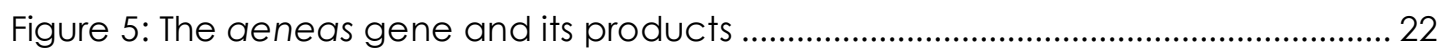

Figure 6: Conservation of the Aen proteins .................................................................. 53

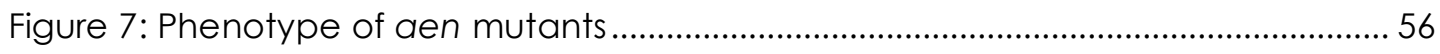

Figure 8: Rescue of PGC transmigration phenotypes in aen ${ }^{2}$ mutants ........................... 58



Figure 10: Whole mount antibody staining with the anti-Aen antibody ..........................66

Figure 11: The subcellular localization of Aen during blastoderm cellularization ..........67 67

Figure 12: Co-localization of Aen with RE and Golgi ......................................................... 70

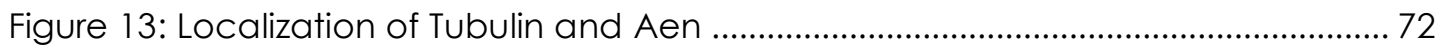

Figure 14: Biochemical interaction studies with potential interactors of Aen ............... 75



Figure 16: Colocalization of Aen with interacting partners............................................... 82

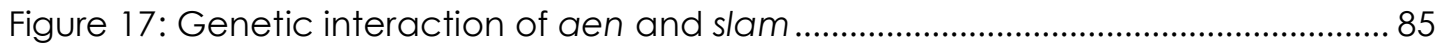

Figure 18: Velocity of membrane invagination in aen ${ }^{2}$ mutants ..................................... 88

Figure 19: Cuticle phenotypes in aen-slam double mutants ............................................ 90 


\section{Tables}

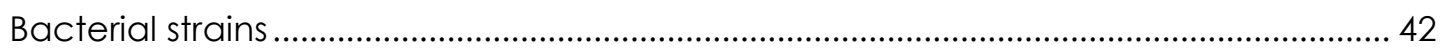

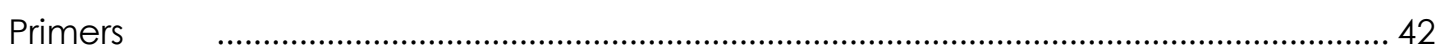

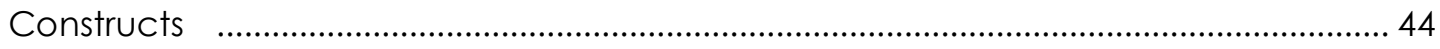

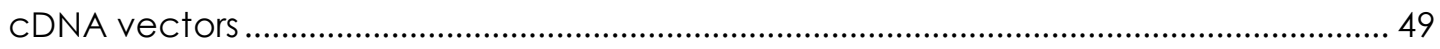

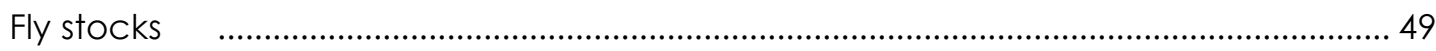

Antibodies used for Western blot detection and co-immunoprecipitation................... 50

Antibodies used in immunohistological stainings.................................................................. 50 


\section{Abbreviations}

aa

AAJ

Arp

BAJ

bp

BSA

c-DNA

$D A B$

$\mathrm{Da}$

DNA

Drosophila

DTT

EDTA

F-actin

FC

FCS

Gal4

GFP

g

HRP

JAK

k

LB
Amino acids

Apical adhesive junction

actin related protein

Basal adhesive junction

Basepairs

Bovine Serum Albumine

copy-DNA

Diaminobenzidine

Dalton

Desoxyribonucleic acid

Drosophila melanogaster

Dithiotreitol

Ethylene diamine tetra-acetate

Filamentous actin

Furrow canal

Fetal calf serum

Gal4 Transcription factor

Green fluorescent protein

Gram

Horse radish peroxidase

Janus Kinase

Kilo

Luria Bertani 


\begin{tabular}{|c|c|}
\hline I & Liter \\
\hline LOF & Loss of function \\
\hline$\mu$ & Micro \\
\hline $\mathrm{m}$ & Milli \\
\hline min or ' & Minutes \\
\hline mRNA & Messenger RNA \\
\hline OD & Optical density \\
\hline ORF & Open reading frame \\
\hline PBS & Phospate buffered saline \\
\hline PCR & Polymerase chain reaction \\
\hline PFA & Paraformaldehyde \\
\hline PGC & Primordial germ cell \\
\hline PMG & Primordial midgut \\
\hline RE & Recycling endosome \\
\hline RNA & Ribonucleic acid \\
\hline rpm & Rounds per minute \\
\hline RT & Room temperature \\
\hline RTK & Receptor Tyrosine Kinase \\
\hline SAJ & Spot adhesive junction \\
\hline SDS & Sodium dodecyl sulfate \\
\hline $\mathrm{sec}$ & Seconds \\
\hline SGP & Somatic gonadal precursor cell \\
\hline STAT & Signal transducer and activator \\
\hline TGF & Transforming growth factor \\
\hline UAS & Upstream activating signal \\
\hline ZA & Zonula adherens \\
\hline
\end{tabular}




\section{Abstract}

The communication and mutual influence of epithelia and migratory cells is a field of high scientific and medical relevance. Many cellular processes underlying the establishment of cell polarity and adhesion are implicated in migratory cell movement including metastasis in cancer.

Primordial germ cells (PGCs) prematurely cross the epithelial barrier of the somatic blastoderm during its cellularization in Drosophila mutants of the aeneas (aen) gene. The objective of this work was the characterization of the biological and molecular function of the Aen protein.

In this thesis I show that Aen is required in the somatic cells for the integrity of the blastoderm epithelium. Aen co-localizes with secretory vesicles and with proteins of the dynactin complex required for vesicular transport. Biochemical interaction experiments as well as coimmunoprecipitation experiments reveal that Aen interacts with Arpl, an important component of the dynactin complex, suggesting a function of Aen during secretory vesicle transport. Furthermore Aen interacts biochemically and functionally with Slow as molasses, which is required for blastoderm cellularization and guided PGC migration.

In summary, the results suggest that Aen encodes a novel conserved adaptor of the Dynactin complex, required for Slow as molasses (Slam) transport or function in a process essential for the epithelial integrity that consequentially inhibits PGC mismigration. Therefore the aen mutation opens new ways to address important questions on tissue invasion by migratory cells. 


\section{Introduction}

Cell migration and the generation of structural and osmotic barriers are important aspects of life. In the eukaryotic domain the means of locomotion of unicellular organisms have laid the foundation for the mode of cell migration found in multicellular organisms (reviewed in Schneider and Haugh, 2006). Even though the molecular mechanisms, that allow the movement of a cell are conserved to a high degree, the implications of migration and the molecular mechanisms underlying the guidance of migrating cells have changed considerably with the transition from protozoan to metazoan life. In multicellular organisms, cell migration no longer serves the purpose of locomotion. Nevertheless, cell migration is a prerequisite of metazoan life, being required for the development and maintenance of a structured and functional organism. During embryogenesis, the formation of the mesoderm (e.g. in Sea urchin, Drosophila, chicken) and the closure of the neural tube (chicken) are exemplary for the involvement of cell migration in morphogenetic movements (reviewed in Gilbert, 2006). In adult organisms, cell migration is involved in wound healing (fibroblasts; reviewed in Li et al., 2004) and immune response (leucocytes; reviewed in Zen and Parkos, 2003).

Accordant to its importance for the functioning of a multicellular organism, aberrant cell migration is implicated in pathological processes, the most prominent example being the spreading of metastases from malignant tumors (reviewed in Sahai, 2005).

The formation of epithelia acting as physical barriers is a second important characteristic of multicellular organisms. Apart from protecting the organism from physical stress, pathogens, desiccation 
or osmotic pressure (epidermis, gut epithelia, lung/trachea), other important functions like gas exchange (lung, trachea), the resorbtion of nutrients (gut epithelium), the transport of metabolites (blood vessel system) and finally the excretion of waste products (kidney, malpighian tubules in Drosophila) are mediated by epithelia (reviewed in Williams et al., 1995; Nation, 2002). To exert these functions, epithelial cells share two main characteristics, apico-basal polarity and tight adhesion. These attributes are essential for epithelial integrity and function (reviewed in Tepass et al., 2001).

In the concert of morphological movements during the development of a higher metazoan organism, the vital processes of cell migration and the maintenance of epithelia must be reconciled. Mechanisms have evolved that regulate the passage of cells through tissues and that allow for rearrangement of adhesive structures in between epithelial cells. However many of the processes and factors involved are not fully understood.

In Drosophila, the formation primordial germ cells (PGCs) requires the positioning of distinct maternally synthesized factors in the oocyte. PGCs, formed by budding out form the posterior embryonic pole, are the first individual cells of the embryo (reviewed in Williamson and Lehmann, 1996). After the somatic lineages have cellularized, the PGCs move from their point of origin, towards the gonadal primordia. Thereby they actively cross epithelial barriers and other tissues. Several mutations, which affect these processes on various levels, have been characterized. Therefore, early embryogenesis of Drosophila melanogaster provides highly established and well accessible experimental system to analyze cell migration, the formation and maintenance of epithelial tissues as well as the interplay between the two cell types. 


\subsection{Drosophila development}

\subsubsection{Oogenesis in Drosophila melanogaster}

Drosophila oogenesis shows the characteristics of polytrophic meroists (reviewed in Snodgrass, 1993). Nutrients and essential contents of the future embryo are synthesized and imported into the oocyte from germline derived nurse cells and somatic cells (reviewed in Snodgrass, 1993). During this process also the axes of the future embryo are defined by positioning of distinct maternally synthesized factors in the oocyte. This process is a prerequisite for PGC formation (reviewed in Jin and Xie, 2006).

Drosophila ovaries consist of about 16 ovarioles, which are surrounded by connective- and muscle tissue. Two to three germ line stem cells (GSC) are located in the germarium of each ovariole at its most anterior tip (figure 1a) (reviewed in Huynh and St Johnston, 2004). The stem cell division gives rise to a GSC and a cystoblast, which then undergoes 4 incomplete divisions resulting in an egg follicle of 16 connected cells, the cystocytes (figurelb). Egg follicles leave the germarium in posterior direction, forming individual egg chambers, which are ensheathed by somatic follicle cells (figure la).

During cystocyte divisions a continuous network of microtubules (MT) is established, that extends into all cystocytes through cytoplasmic bridges, the ring canals (Storto and King, 1989; Navarro et al., 2001; Lin et al., 1994; de Cuevas and Spradling, 1998). The pattern of divisions results in two cystocytes with four ring channels, the pro-oocytes (figure 1b; Lin et al., 1994; Lin and Spradling, 1995). One of the prooocytes adopts oocyte fate whereas the other pro-oocyte and the remaining cystocytes develop into nurse cells. The differentiation of oocyte fate is dependent on the on MT based transport mediated by dynein and the dynactin complex (Theurkauf et al., 1993; Grieder et 
al., 2000; Huynh et al., 2001; Riechmann and Ephrussi, 2001). The dynactin complex which mainly acts as a cofactor of the motor protein complex dynein comprises three subcomplexes, a rod-shaped scaffold consisting mainly of Arpl (Schafer et al., 1994; Bingham and Schroer, 1999; Schroer and Sheetz, 1991; Gill et al., 1991; Schafer et al., 1994; Schroer et al., 1994; Schoer et al., 1996; Imai et al., 2006), a shoulder and a sidearm complex, containing Dynamitin and a dimer of the protein Glued (reviewed in Schroer, 2004; Imai et al., 2006). BicaudalD (BicD) and Egalitarian (Egl) (Mohler and Wieschaus, 1985; Mohler and Wieschaus, 1986; Navarro et al., 2004), are both associated to the dynein motor complex and act as cargo receptors, but are also required for the polarization of the MT cytoskeleton (reviewed in Pearson and Gonzalez-Reyes, 2004; Riechmann and Ephrussi, 2001). When the functions of the dynactin complex and associated factors are affected, defects in oocyte differentiation occur. The treatment of ovaries with MT depolymerizing drugs leads to the failure of oocyte differentiation, resulting in the formation of egg follicles containing 16 nurse cells and no oocyte (Koch and Spitzer, 1983; Theurkauf et al., 1993). Similar phenotypes are observed in mutants of arp 1, bicD and egl (Haghnia et al., 2007; Mohler and Wieschaus, 1985; Mohler and Wieschaus, 1986; McGrail et al., 1997; Mach and Lehmann, 1997; Riechmann and Ephrussi, 2001; Navarro et al., 2004).

After oocyte specification, the nurse cells produce large quantities of organelles, metabolites, mRNAs and proteins, which are transported into the oocyte (reviewed in Spradling, 1993). Also the somatic follicle cells surrounding the oocyte are involved in the synthesis and import of nutrients. The lumen of a mature egg is densely populated by yolk 


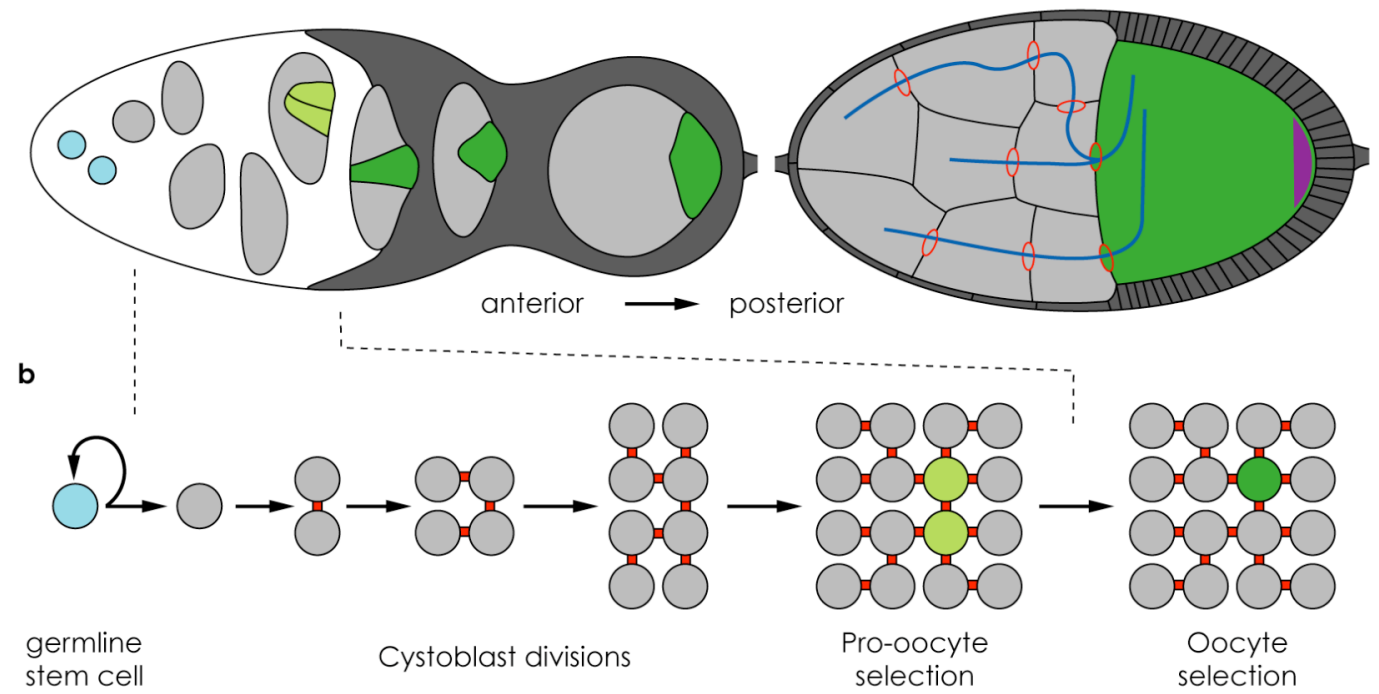

Figure 1: Oogenesis of Drosophila melanogaster. a) Graphical representation of the development of egg follicles inside of a Drosophila ovariole. The development of egg follicles is shown advancing from anterior to posterior. The scheme on the left side shows the germarium at the anterior tip of the ovariole. On the right side, an egg chamber at stage 9 is depicted displaying the ring canals connecting the cells in the follicle as well as the microtubule (MT) bundles mediating the transport from the nurse cells to the oocyte and the localization of oskar (osk). b) Diagram of cystocyte divisions from the germline stem cell to the 16-cell follicle. The pattern of ringchannels connecting the cystocytes and the process of oocyte selection are depicted. Color scheme: light blue (germline stem cells), light grey (cystocytes/nurse cells), light green (pro-oocytes), dark green (oocyte), red (ring canals), dark blue (MT), dark grey (somatic follicle cells), purple (osk). (Concept of drawing adapted from Huynh et al. 2001).

particles and lipid droplets (LD) (Spradling, 1993). The sum of compounds transported into the oocyte is termed maternal contribution (Spradling, 1993).

The majority of factors of the maternal contribution, for example hunchback (hb) mRNA (Lehmann and Nüsslein-Volhard 1987), are ubiquitously distributed in the oocyte. In contrast, factors required for the determination of the anteroposterior and dorsoventral axes of oocyte exhibit specific localization. This localization is based on the activity of the TGF- $\alpha$ signaling factor Gurken (Grk) (Schüpbach, 1987; Cooperstock and Lipshitz, 2001; Roth, 2003; Van Buskirk and Schüpbach, 1999). grk mRNA and protein are transported in a dynactin-dependent manner (Januschke et al., 2002; Caceres and Nilson, 2005; Clark et al., 2007). Grk signaling between the oocyte and the adjacent somatic follicle cells, first at the posterior end of the oocyte, then shifting in dorsoanterior direction, is the founding event of anteroposterior and dorsoventral body axis determination (reviewed in 
Gonzalez-Reyes et al., 1995; Anderson and Nüsslein-Volhard, 1984, Poulton and Deng, 2006). During this process the MT cytoskeleton of the oocyte undergoes several steps of repolarization (Gonzalez-Reyes et al., 1995; Theurkauf et al., 1992), which enables the transport of material from the nurse cells into the oocyte and the localized deposition of distinct mRNAs required for the formation of the anteroposterior body axis of the embryo. These factors include the posterior localization of oskar (osk) mRNA (Lehmann and NüssleinVolhard, 1986) and the anterior localization of bicoid mRNA (bcd) (Fröhnhofer and Nüsslein-Volhard, 1986; Weil et al., 2006). Transport and positioning of osk and bcd are MT-dependent (Pokrywka and Stephenson, 1991; Pokrywka and Stephenson, 1995; Clark et al., 2007). Localization of osk mRNA at the posterior pole, defines the position of the pole plasm, a cytoplasmic region containing several factors in addition to Osk protein, including the RNA helicase Vasa (Vas) (Hay et al., 1988) and nanos (nos) mRNA (Wang and Lehmann, 1991; Ephrussi et al., 1991; Lehmann and Nüsslein-Volhard, 1991). During embryogenesis the contents of the pole plasm will define where the PGCs are formed (reviewed in Hyunh and St Johnston, 2004).

\subsubsection{Early embryogenesis}

Based on prominent morphological characteristics, which can easily be identified in the developing embryo, the embryogenesis of Drosophila has been divided into a series of distinct developmental stages (reviewed in Foe et al., 1993; Campos-Ortega and Hartenstein, 1985). The Drosophila embryo forms a plasmodium during the first stages of embryogenesis. After fertilization, fast mitotic divisions occur, which lead to the establishment of a large plasmodium. During embryonic stage 3 (figure 2c), 5 to 10 nuclei migrate into the pole plasm located at the posterior pole of the embryo. The nuclei residing in the pole plasm form the first individual cells of the embryo by 
a

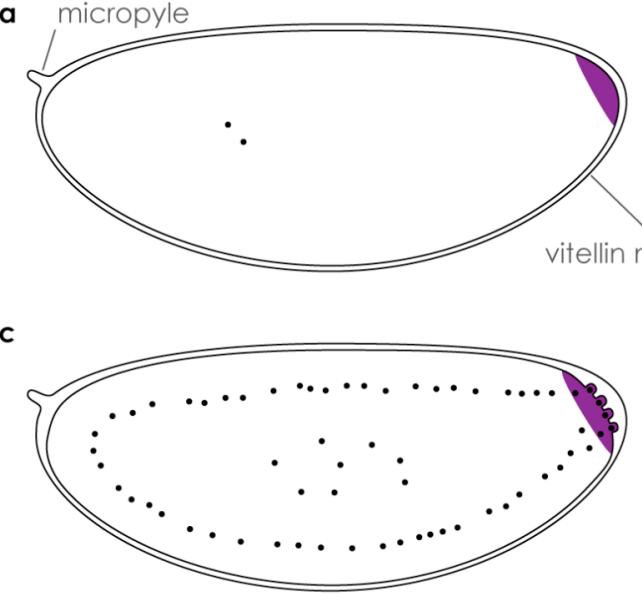

b

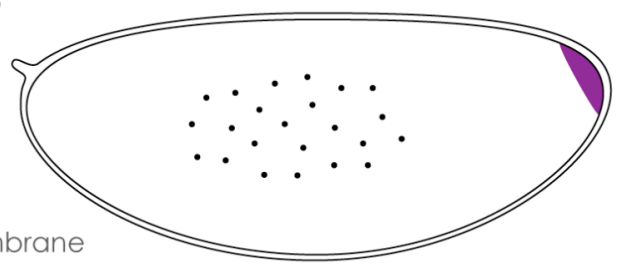

d

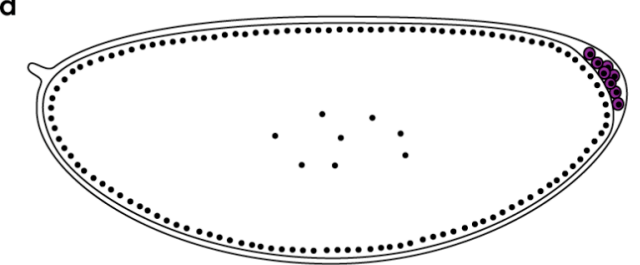

Figure 2: Syncytial stages of embryonic development. a) Stage 1: Pronuclear fusion and mitosis 1. b) Stage 2: Mitotic cycles 2-8, nuclei exhibiting a hollow monolayer shell distribution. c) Stage 3: Mitotic cycles 9-10, nuclei reach the pole plasm, polar budding, formation of the PGCs. d) Stage 4: Mitotic cycles 11-13, alignment of somatic nuclei at the embryonic cortex, formation of the syncytial blastoderm. Color scheme: black (nuclei), blue (stages 1 to 3: pole plasm; purple (stage 4: PGCs). (Drawings after Foe et al., 1993)

budding out from the posterior pole. The cells formed in this process are the PGCs which proliferate and give rise to about 40 cells in later stages (Williamson and Lehmann, 1996). During this stage of development, the Osk protein (see section 1.1.1) acts as a determinant of PGC fate. Ectopic localization of Osk is sufficient to induce ectopic PGC formation in respective positions and in osk loss of function, no PGCs are formed (Ephrussi and Lehmann, 1992). The PGCs strongly differ from the somatic tissue formed at stage 5 in morphology, protein composition and gene activity (see section 1.1.1 and 1.1.3; reviewed in Strome and Lehmann, 2007). Several factors including RNAs (e.g. pole granule component (pgc)) and proteins (e.g. Osk and Vas) are found exclusively in the PGCs (see section 1.1.1 and 1.1.2; reviewed in Strome and Lehmann, 2007). PGCs are transcriptionally silent during stages 3 to 9, which is critical for their survival and the preservation of their germ cell fate (reviewed in Strome and Lehmann, 2007). The polar granules (PGs) are specialized structures in the PGCs, which are strongly enriched in maternal factors such as Osk and Vas (Lehmann and Nüsslein-Volhard, 1986; Hay et al., 1988). Components of the PG are involved in maintaining the 
transcriptional repression in the PGCs and for the posterior morphogenesis of the embryo (Lehmann and Nüsslein-Volhard, 1986; Hay et al., 1988).

At the beginning of stage 4 (figure $2 d$ ) the majority of nuclei have reached the embryonic cortex forming the syncytial blastoderm. The translation of bcd mRNA at the anterior pole leads to the formation of a gradient of Bcd protein which acts as a transcriptional activator for anterior patterning genes in a concentration dependent way (Burz et al., 1998; Rivera-Pomar and Jäckle, 1996; Struhl et al., 1989). The Vas dependent translation of nos mRNA at the posterior pole (Wang and Lehmann, 1991) (see section 1.1.1), results in the formation of a Nos protein gradient. Nos acts as a translational repressor for the morphogen $\mathrm{Hb}$, thereby inducing the derepression of posterior patterning genes (Tautz, 1988; Lehmann and Nüsslein-Volhard, 1987; Hülskamp et al., 1989; Irish et al., 1989; Struhl, 1989). The formation of these morphogen gradients is an essential step in anteroposterior axis formation. The activity of other signal cascades leads to the establishment of the dorsoventral and terminal patterning (reviewed in Moussian and Roth, 2005; LeMosy, 2003). By mitotic cycle 13, about 6000 nuclei form a monolayer directly underneath the embryonic cortex (Foe et al., 1993). The cortical cytoplasm of the embryo is cleared from yolk and LD by MT-dependent transport (Welte et al., 1998; reviewed in Jäckle and Jahn, 1998). The PGCs form a cluster of cells localized at the posterior pole atop the syncytial blastoderm.

\subsubsection{Blastoderm cellularization}

After the formation of the PGCs at the posterior pole is finished, the cellularization of the somatic blastoderm begins (reviewed in Lecuit, 2004). Directly after nuclear cycle 13, marking the beginning of embryonic stage 5, the formation of somatic buds occurs (figure $3 a$ and c). These protuberances of the plasma membrane, which consist 
of a arrays of villous projections (VPs) lined with F-actin, are localized atop of the spherical blastoderm nuclei (Lecuit et al., 2002) (figure 3c). During stage 5-1, the plasma membrane forms hexagonal furrows that synchronously invaginate and vertically enclose the individual cortical nuclei. The leading front of the coherent invaginating membrane structure has been termed furrow canal (FC) (Fullilove et al., 1971). FC formation is initiated and accompanied by reorganization of the actin cytoskeleton (figure $3 \mathrm{c}$ and $\mathrm{d}$ ). While the cortical F-actin concentration decreases, the FC becomes densely populated by actin filaments (Warn et al., 1986) forming contractile rings (Warn et al., 1980; Miller et al., 1995). The protein composition of the FC exhibits great similarity to the contractile apparatus formed during standard cytokinesis. It includes Chickadee, Drosophila Profilin, and the motor proteins Zipper and Spaghetti Squash, the Drosophila homologs of Myosin II heavy and light chain respectively (Young et al., 1993; Karess et al., 1991). Concomitant to the formation of the FC and adjacent to it, the basal adherence junction (BAJ) is formed, which connects the membranes of neighboring cells during their cellularization (Hunter et al., 2000; Lecuit et al., 2002). Its main functional entities are the proteins Shotgun (Shg) (Oda et al., 1994, Tepass et al., 1996), the Drosophila homolog of E-Cadherin, which mediates homophilic interactions between adjacent membranes in a $\mathrm{Ca}^{2+}$ dependent manner, $\alpha$ Catenin (Oda et al., 1993) and Armadillo (Arm) (Peifer and Wieschaus, 1990), the Drosophila $\beta$-Catenin, which acts as a linker between Shg and the actin cytoskeleton. As cellularization proceeds, the BAJs are translocated in basal direction (Lecuit, 2004).

The cortical nuclei, that have maintained a spherical shape during the syncytial stages, start to elongate vertical to the embryonic surface (Lecuit, 2000; Knoblich, 2000) increasing their length about 2,5 fold (Brandt et al., 2006). When the ingressing FC reaches half of the 
a
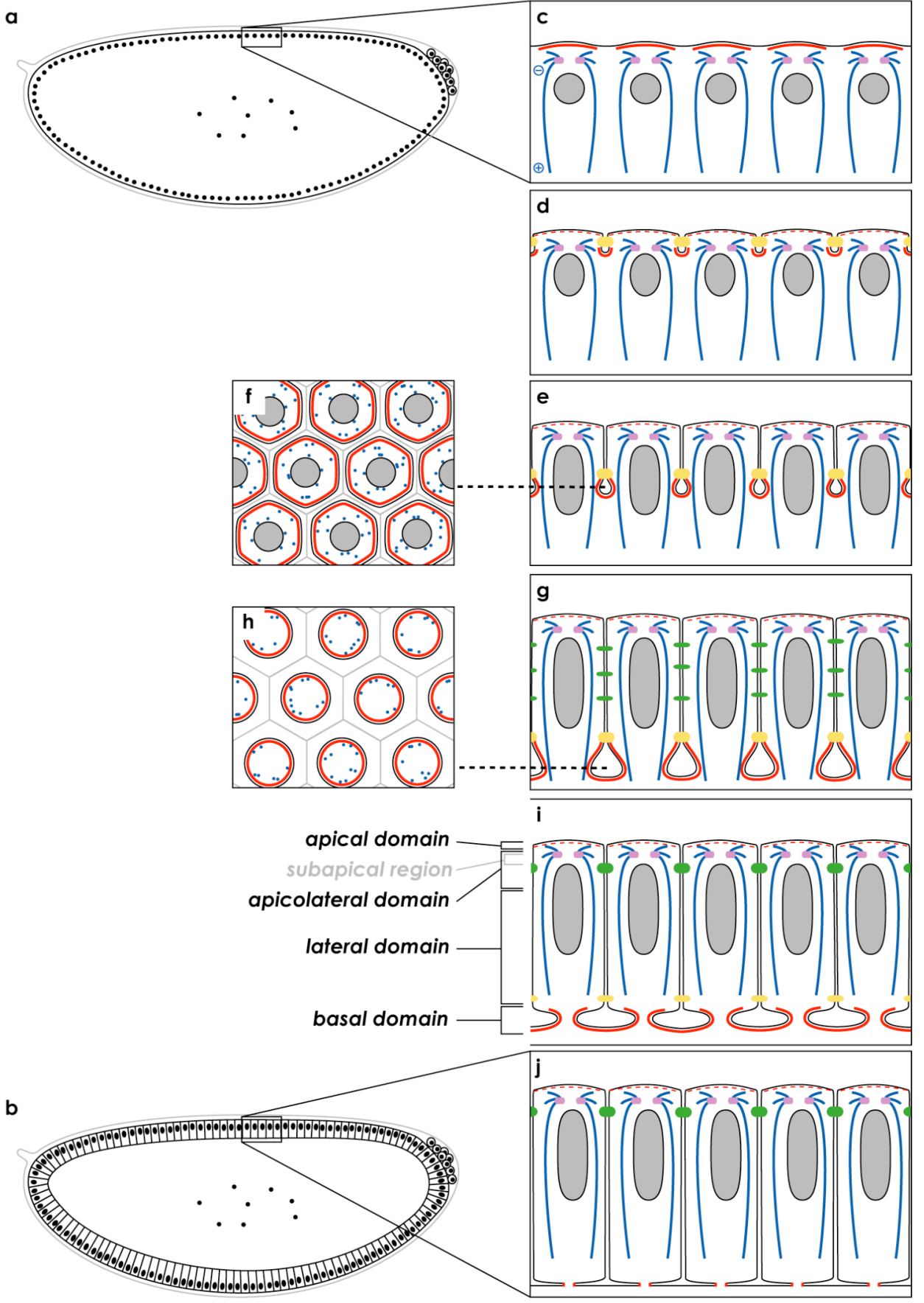

Figure 3: Blastoderm cellularization. Cross sections a)-e), g), i)-j). Grazing sections f), h). a) Syncytial blastoderm, stage 4 to 5-1. b) Cellular blastoderm c) Somatic budding; beginning of stage 5-1; villous projections form above cortical nuclei. d) Formation of the FC (FC); actin and myosin II, localized to the leading front of the FC, form hexagonal shaped rings (see also f)); invagination of membranes between the nuclei; nuclear elongation; formation of the basal adhesive junction (BAJ). e) and f) Slow furrow progression; Beginning of stage 5-2. g) and h) Fast furrow progression; formation of the apical spot adhesive junctions (SAJ); contraction of actin-myosin ring begins; Beginning of stage 5-3. i) Condensing of SAJs into apical adhesive junction (AAJ); contraction of actin-myosin ring leading to the pinching off of individual cells. j) End of cellularization; formation of the cellular blastoderm; resolving of BAJ. Color scheme: grey (nuclei), red (actin filaments), dark blue (MT), purple (Centrosomes), yellow $(B A J)$, green (SAJ and AAJ). 
nuclear length embryonic stage 5-2 begins (figure $3 e$ and $\mathrm{f}$ ). While the inward progression of the FC commences at slow rates, the nuclei finish their elongation. When the FC reaches the basal end of the nuclei, at stage 5-3, the invagination speed increases (Lecuit et al., 2002). During this fast phase of membrane invagination (figure $g-i$ ), the contractile rings at the $\mathrm{FC}$ begin to contract. After the progression of the FC comes to a halt, this contraction finishes (figure 3j) leading to an incomplete pinching of individual cells which leaves a thin connection to the underlying yolk, the cytoplasmic stalk (Foe et al., 1993).

During the entire process of cellularization, the surface of the plasma membrane surrounding the embryo and separating the nuclei increases approximately 23 fold. The invagination of the membranes during cellularization is only partially achieved by stretching of preformed membrane reservoirs. Instead it is mainly brought about by integration of de novo synthesized material (Lecuit et al., 2002; Burgess et al., 1997). This process provides the force that propels the ingression of the membranes. In the onset of membrane invagination, gradually 4 membrane domains are formed, the apical-, the apicolateral-, the lateral- and the basal membrane (Lecuit and Wieschaus, 2000; Tepass et al., 2001) (figure 3i). The region, at which newly synthesized material is integrated shifts from the apical membrane during stages 5-1 and 52 to the apicolateral membrane during fast furrow progression at stage 5-3. The extension of the membrane thereby coincides with the generation of specialized membrane areas such as adhesive junctions (Lecuit et al., 2002). This finding indicates that the membrane, as it is formed, undergoes a polarized remodeling driven by the targeted integration of membrane material. Supporting this model, many factors and structures involved in intracellular transport are involved in cellularization. 
The MT, even though not directly involved in propelling the invagination of the newly formed membranes are required for the process of cellularization. Inhibiting the formation of MTs by injection of anti-Tubulin antibodies leads to a complete inhibition of FC progression (Warn et al., 1987).

In mutants of the gene lava lamp (IVa)(Sisson et al., 2000; Papoulas et al., 2005), cellularization is inhibited as well. Lva is a scaffold protein that connects Golgi particles to the dynein motor complex enabling the transport of Golgi vesicles along MTs.

Nuclear fallout (Nuf) (Rothwell et al., 1998) and the small GTPase Rabl1 are localized to the recycling endosomes (RE) (Ullich et al., 1996; Sasamura et al., 1997). nuf and rab 11 mutants exhibit defects in vesicle fusion to the membrane region of the prospective $\mathrm{FC}$ and redistribution of actin filaments (Riggs et al., 2003). In both mutants, malformation of FC can be observed, leading to the formation of multinucleate cells indicating that the centrosome-associated REs, which are localized directly apical of the nuclei, are involved in the trafficking of membrane and cytoskeletal components during cellularization.

The activity of the formin Diaphanous (Dia) and RhoGEF2 is required for the assembly of the actin structure prior to formation of the FC. In dia and RhoGEF2 mutants, the concentration of F-actin at the FC is reduced. Additionally its morphology is altered and exhibits enlarged membrane invaginations filled with cytoplasmic blebs. In some areas the FC does not form leading to multinucleate cells (Großhans et al., 2005).

Nullo is involved in the organization of the actin cytoskeleton of the FC (Simpson et al., 1990; Postner and Wieschaus 1994; Hunter et al., 2002). Furthermore, it is required for the establishment and stabilization of the 
BAJ (Hunter and Wieschaus, 2000). In nullo mutants the formation of the BAJ is compromised, leading to a partial failure of cellularization and formation of multinucleate epithelial cells (Simpson et al., 1990; Hunter et al., 2002). Serendipity- $\alpha$ (Sry- $\alpha)$, which colocalizes with Nullo (Vincent et al., 1985; Schweisguth et al., 1990; Ibnsouda et al., 1993), acts in conjunction with Nullo to maintain the cytoskeleton at the FC.

The protein Slow as molasses (Slam) is required for the proper localization of cytoplasmic myosin and the adhesion factor D-PATJ (Bhat et al., 1999; Pielage et al., 2003). In slam null mutants the FC and adjacent BAJ (Lecuit et al., 2002, Stein et al., 2002) are malformed. Consequentially, the cellularization is delayed and stops prematurely. This observation indicates that the Slam protein is involved in the formation of the FC and lateral BAJ (Lecuit et al., 2002), during cytokinesis. Two models have been suggested, how Slam could affect the formation of these domains. It might either act as a scaffolding protein bringing D-PATJ and other factors in contact to each other or it might be involved in directing membrane transport inferring specificity to vesicle fusions taking place in the respective membrane areas. The latter of the models is supported by the finding that Slam loss of function alters the kinetics of membrane invagination (Lecuit et al., 2002) and that Neurotactin (Nrt) (Hortsch et al., 1990), a heterophilic adhesion factor, which is localized to the apical, apicolateral and lateral membrane domains in wildtype, is mislocalized to the apical cytoplasm in slam loss of function. Additionally, in experiments tracing the incorporation of membranes into a fluorescently labeled plasma membrane, it was found that, while the sites of membrane incorporation are closely restricted to the apical and apicolateral plasma membrane in wildtype, this distinction does not exist in Slam lack of function (Lecuit and Wieschaus, 2000). However both postulated models for the function of Slam might apply (Lecuit et al., 2002). 
During fast furrow progression, at stage 5-3, apical spot adhesive junctions (SAJ) are generated which fuse to give rise to the apical adherence junction (AAJ) and exhibit a similar composition as the BAJ. During gastrulation, this structure forms a continuous Zonula Adherens (ZA) connecting the epithelial cells (Müller and Wieschaus, 1996; Tepass, 1996). The joining of the SAJs into a continuous AAJ is in part modulated by the Nullo protein, which appears to protect adhesion factors of the BAJ of being recruited into the AAJ. When nullo is overexpressed, no AAJ is formed. Instead the individual SAJS are stabilized (Hunter and Wieschaus, 2000).

The formation of the AAJ, which develops into the ZA after completion of cellularization, is dependent on three protein complexes that define the apicobasal polarity of the forming epithelial cells (reviewed in Johnson and Wodarz, 2003). Components of the Bazooka complex, consisting of Bazooka (Baz) (Müller and Wieschaus, 1996, Knust et al., 1996), Drosophila atypical Protein Kinase C (DaPKC) (Wodarz et al., 2000) and DPar-6 (Tomancak et al., 2000), are localized to the subapical region of the blastoderm cytoplasm, apical of the ZA (Wodarz et al., 2000; Petronczki und Knoblich, 2001; Bachmann et al., 2001; Hong et al., 2001). This localization requires the presence of Arm (Müller and Wieschaus, 1996). In arm mutants the formation of adhesive structures is affected (Müller and Wieschaus, 1996) leading to the disintegration of the blastoderm epithelium during gastrulation. In loss of function of baz, markers for apical membranes and the components of the BAJ are mislocalized and the formation of the ZA is compromised leading to malformation of the blastoderm epithelium, similar to the phenotype observed in arm mutants, and to extensive cell death at later stages (Bilder et al., 2003; Müller and Wieschaus, 1996). Similar phenotypes can also be observed for loss of function mutants of other components of the complex (Wodarz et al., 2000; Petronczki and Knoblich, 2001). 
The Crumbs complex consists of Crumbs (Crb) (Tepass et al., 1990; Wodarz et al., 1995; Wodarz et al., 1993; Jürgens et al., 1994), Stardust (Sdt) (Müller and Wieschaus, 1996; Wodarz et al., 2000), DLin-7 (Bachmann et al., 2004) and D-PATJ (Pielage et al., 2003). Like the Baz complex, it is localized to the subapical region of the epithelial membranes. Crb is a transmembrane protein that acts as a structural component of the ZA. Mutants affecting the crumbs complex exert adhesion phenotypes similar to the mutants of arm or constituents of the Baz complex. The requirement of Baz for $\mathrm{Crb}$ localization indicates that the Crb complex acts downstream of Baz (Bilder et al., 2003; Müller and Wieschaus, 1996; Müller, 2000). The third protein complex involved in the generation of apicobasal polarity is the Discs large complex, which acts downstream of the Baz and Crb complexes and encompasses the factors Discs large (Dlg) (Perrimon, 1988), Scribble (Scrib) (Bilder et al., 2000) and Lethal giant larvae (L(2)gl) (Strand et al., 1994). Similar to mutations affecting the Baz or Crb complex, the formation of the ZA is compromised in mutants of the Dlg complex (Bilder et al., 2003), however no ectopic cell death is observed in this case.

The contraction of the contractile ring during fast furrow progression is timed by a mechanism involving the factor Bottleneck (Bnk). In mutants of bnk, this contraction happens too early during stage 5-2 leading to constrictions pinching the cortical nuclei (Schejter and Wieschaus, 1993; Theurkauf, 1994).

After the invagination of the plasma membranes is finished and the FC has fully contracted, individual cells have formed and establishment of the cellular blastoderm is complete. The LDs are imported into the newly formed epithelial cells through the cytoplasmic stalks. This transport is again MT-dependent (Welte et al., 1998; reviewed in 
Jäckle and Jahn, 1998). The breakdown of the BAJs marks the end of cellularization (Hunter et al., 2000) (figure 3 b and j).

\subsubsection{PGCs in embryogenesis}

When the PGCs reach the location of the prospective gonad, they differentiate into gonadal stem cells, which give rise to sperm or eggs in the imagos (reviewed in Williamson and Lehmann, 1996).

The guidance of PGC migration in vertebrates is to a great extend mediated by a simple system consisting of the chemokine SDF1 and its receptor CXCR4 (Doitsidou et al., 2002; Molyneaux et al., 2003). In Drosophila, which contains neither of these factors, the guidance of PGC migration is dependent on a variety of factors and pathways.

During embryonic stages 4 and 5, the PGCs reside at the place of their formation, at the posterior pole of the somatic blastoderm. During this phase, the PGCs are shaped spherically, exhibit a uniform layer of cortical actin and do not form pseudopodia (Jaglarz and Howard, 1995). The JAK/STAT signal transduction pathway appears to affect PGC migration, proliferation and maintenance at blastoderm stage (Li et al., 2003). Overactivation of the pathway during early embryogenesis leads to preterm transmigration at of PGCs at the cellular blastoderm stage (Binari and Perrimon, 1994; Hou et al., 1996). After blastoderm cellularization (see section 1.1.3), the embryo enters gastrulation. The portion of primary epithelium on which the PGCs reside (Turner and Mahowald, 1976) is part of the primordial midgut anlage (PMG) (figure 4a).

At stage 6, the ventral blastoderm epithelium stretches along the longitudinal axis of the embryo. This process, the germ band extension (figure $4 b-d$ ), leads to a dorso-anterior shift of the PMG. During this movement, the PGCs are passively carried along with the underlying PMG. Even though the PGCs do not actively migrate, extensive 


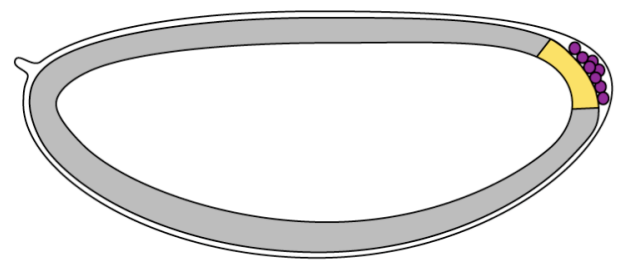

c

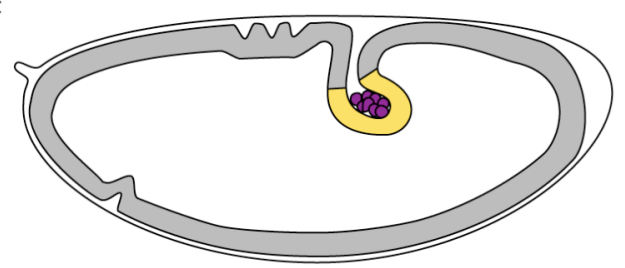

f

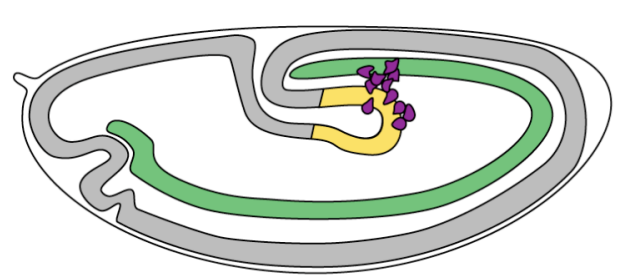

g

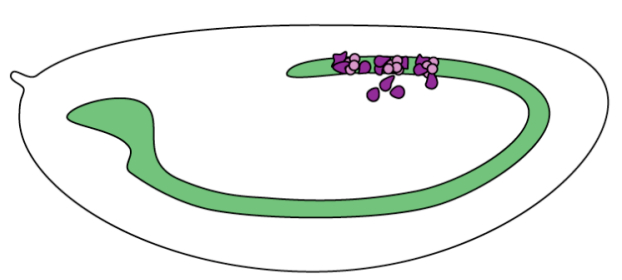

i

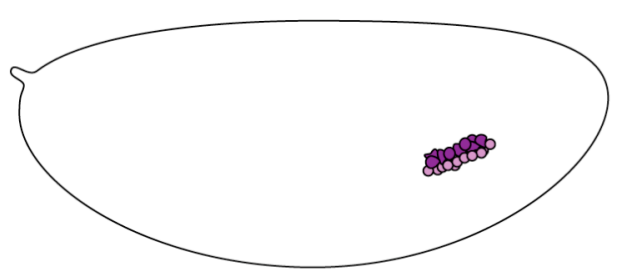

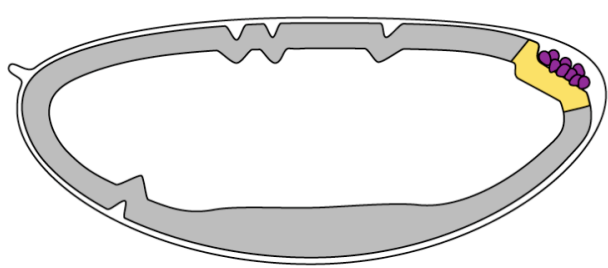

d

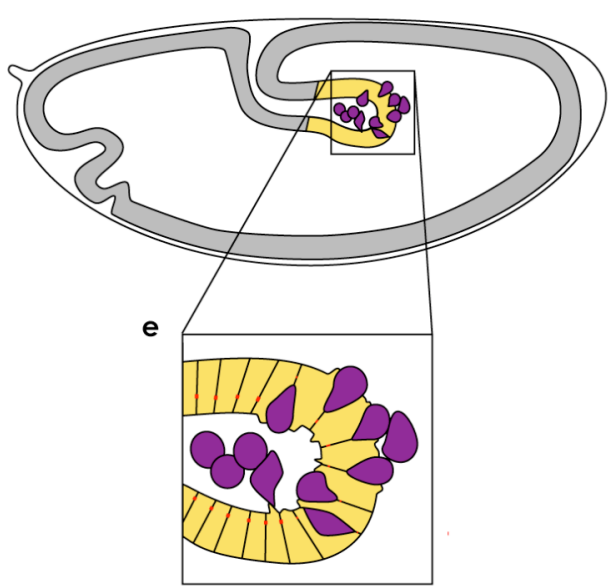

h

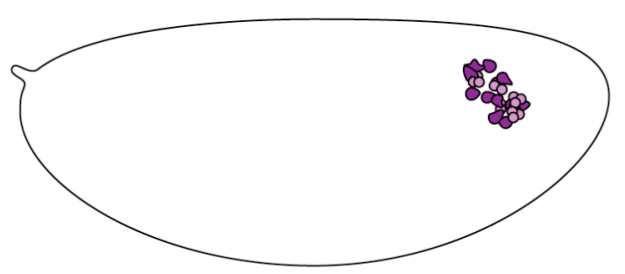

j

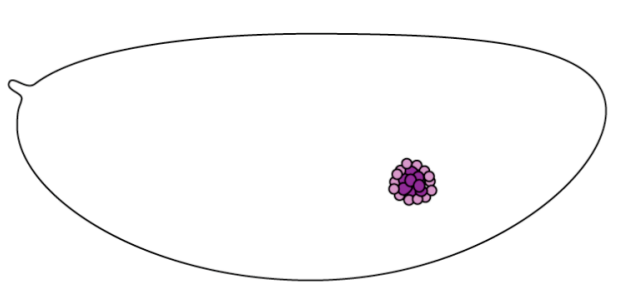

Figure 4: Embryonic stages in respect to PGC development. a) Stage 5-3; cellular blastoderm. b) Stage 6; beginning of germ band extension. C) Stage 8; germband extension; invagination of the PMG pocket. d) and e) Stage 9; migration of PGCs through the epithelium of the PMG pocket. f) Stage 10; reorientation of PGCs on the PMG pocket, migration towards the mesoderm. g) Stage 11; migration into the visceral mesoderm; attachment. h) Stage 12; alignment with SGP clusters; germ band retraction. i) Stage 13; clustering of PGCs and SGPs. j) Stage 14; gonad coalescence. Color scheme: gray (primary epithelium), purple (PGCs), yellow (PMG), red (AJ), green (mesoderm), light purple (SGPS). (Drawings after Campos-Ortega and Hartenstein, 1997)

changes in their morphology can be observed. The PGCs form pseudopodia, making contacts to the PMG and other PGCs (Jaglarz and Howard, 1995). These changes are also reflected in the cytoskeletal morphology. The cortical actin levels detected around the pseudopodia are reduced and focal accumulations of F-actin become visible in the cytoplasm (Jaglarz and Howard, 1995). In the 
onset of germ band extension the PMG invaginates (figure 4C), resulting in the formation of the PMG pocket. With the convolution of the PMG, the PGCs are passively internalized and remain tightly packed in the blind end of the PMG pocket until the end of stage 8 (Skaer, 1993; Jaglarz and Howard, 1995).

Embryonic stage 9 marks the transition to active migration of the PGCs (figure 4d and e). They intercalate the epithelial cells of the PMG pocket and transmigrate the epithelium. Several studies have shown that this process is not exclusively mediated by the PGCs but that it also requires changes in the epithelial cells of the PMG (Jaglarz and Howard 1994; Callaini et al., 1995; Warrior, 1994).

During PGC transmigration, the PMG epithelium undergoes morphological changes, which can be observed also in the absence of PGCs (Jaglarz and Howard 1994; Callaini et al., 1995). The epithelial cells of the PMG form pseudopodia on their luminal surface, contacting the PGCs. Upon contact with the PGCs, discontinuities in the adhesive belts of neighboring PMG cells occur, permitting the transit of the PGCs (Jaglarz and Howard, 1994). The PMG epithelium therefore appears to possess specialized traits that actively promote the process of transmigration. In good agreement to this notion is the finding that the PGCs are trapped the PMG pocket of serpent (srp) and huckebein (hkb) mutants. In these mutants, the PMG epithelium is misdetermined to become hindgut epithelium (Reuter, 1994; Warrior, 1994; Jaglarz and Howard, 1994; Brönner and Jäckle, 1996; Moore et al., 1998). A similar PGC migration phenotype has been shown for mutants of the G-Protein coupled receptor Trapped in endoderm 1 (Tre1) (Kunwar et al., 2003). The downstream target of Trel is the Rho GTPase 1, which has been suggested to mediate cytoskeletal rearrangements occurring specifically in migrating PGCs (Kunwar et al., 2003). 
Trel has also been hypothesized to act in conjunction with components of the Wunen signaling pathway (Kunwar et al., 2003). The timing and directionality of PGC migration during and after their transmigration of the PMG pocket is governed mainly by the Wunen pathway. The proteins Wunen (Wun) (Zhang et al., 1996) and Wunen2 (Wun2) (Starz-Gaiano et al., 2001) are lipid-phosphate phosphatases. It has been shown that both Wun proteins act as receptors for a lipid derived second messenger, which is hypothesized to act as an attractant and survival signal for PGCs and that is modified and internalized by PGCs and somatic tissue alike in a Wun dependent manner (Starz-Gaiano et al., 2001; Renault et al., 2004). Somatic tissues expressing Wun at high levels, such as the ventroanterior part of the PMG, act repellant on the PGCs by depriving their surroundings of the second messenger (Starz-Gaiano et al., 2001). Accordingly, the PGCs, migrating away from the wun expressing tissue, transmigrate the PMG in dorso-posterior direction. Somatic Loss of function of wun leads to mismigration of PGCs (Renault et al., 2004).

After crossing the PMG, the PGCs arrange dorsally of it (figure 4f), next to the visceral mesoderm (Jaglarz and Howard, 1995). During stages 10 and 11, the PGCs partition into two groups which split along the longitudinal axis of the embryo and migrate away from ventral midline in lateral directions. Again wun signaling is implicated in the directionality of PGC migration. wun2 is expressed in an ectodermal domain along the midline and lateral of the mesoderm. The PGCs, avoiding the wun2 expressing tissue migrate in lateral direction to meet the visceral mesoderm (Renault et al., 2004; Kusch and Reuter, 1999).

The protein Slam (see section 1.1.3), which is involved in cellularization, appears to have a function in PGC migration at the stages 10 to 11 as well. Mutations of the slam gene cause mismigration of PGCs after the 
cells have left the PMG. The functional role of Slam in PGC guidance is unclear as its protein levels decrease below detection level shortly after blastoderm cellularization is finished (Stein et al., 2002) a fact that is interpreted by a Slam function during cellularization that affects PGC migration later in development.

Mutations of STAT92E, a major component of the Jak/STAT signal transduction pathway, cause a reduction of PGC number and mismigration of PGCs after the transmigration of the PMG at stage 10. This mismigration leads to a complete loss of gonad formation (Li et al., 2003; Brown et al., 2006).

When the PGCs have left the PMG they migrate in lateral direction and finally enter the visceral mesoderm where they contact the mesodermal somatic gonadal precursor cells (SGPs) (figure $4 \mathrm{f}$ and $\mathrm{g}$ ). The directionality of migration thereby is dependent on several factors including Columbus (Clb) (Van Doren et al., 1998) a HMG CoA Reductase, involved in isoprenoid biosynthesis and the Hedgehog signaling pathway (Hh) (Deshpande et al., 2001; Tabata and Takei, 2004). Factors which are involved in the specification of the mesoderm, Zinc finger homeodomain protein 1 (Zfh-1) (Broihier et al., 1998), the FGF-receptor Heartless (H+I) (Gisselbrecht et al., 1996), FushiTarazu (ftz) (Wakimoto et al., 1984; Duncan et al., 1986) and Oddpaired (Opa) (Jürgens et al., 1984) are as well required for PGC migration (Moore et al., 1998). PGCs and SGPs form loose cell accumulations, which are shifted ventrolaterally in the course of germ band retraction during stages 12 to 13 (figure $4 \mathrm{~h}$ and i). Mutations affecting the development of the gonadal mesoderm like abdominal $A(a b d A)$ (Cumberledge et al., 1992; Karch et al., 1990), abdominal B (abdB) (Celniker et al., 1989), trithorax (trx) (Mazo et al., 1990), clift (cli) (Boyle et al., 1997; Bonini et al., 1993) and tinman (tin) (Bodmer et al., 1993) cause the dissociation of PGCs from the mesoderm during these 
stages, resulting in a dispersed distribution of PGCs (Boyle and DiNardo, 1995; Moore et al., 1998).

During germ band retraction, the accumulations of PGCs and SGPS compact, culminating in the coalescence of the primordial gonads during stage 14 (Moore et al., 1998). In this process a compact cluster of PGCs is formed and enveloped by SGPs (figure 4j). In mutants of the transmembrane protein Fear of intimacy (Foi), the gonad coalescence remains incomplete without affecting the fate of the cells (Van Doren et al., 2003). As the mutation of the adhesion factor shg (see section 1.2.1) causes a similar phenotype in the gonads, (Jenkins et al., 2003) Foi has been suggested to act as a regulator for shg expression or function.

\subsection{The aeneas gene and protein}

The objective of this work was the characterization of the role of the gene aeneas (aen) (Molitor, 2002) during the processes of blastoderm cellularization and PGC migration. The ectopic induction of aen in muscle apodeme precursor cells causes the occurrence of fused muscle fibres with highly abnormal morphology (Molitor, 2002). Also, in contrast to wildtype, muscle fibres crossing the ventral midline occur. This phenotype laid the basis to the further analysis of aen in the group of Dr Gerd Vorbrüggen.

The sequence analysis of the genomic locus of aen, which is located at cytogenetic band 82A6 (Flybase, 1999; http://flybase.bio. indiana.edu/) of the 3rd chromosome, indicated that the gene contains 8 exons and codes for two transcripts which arise from differential promoters initiating the transcription of either exon $1 \mathrm{a}$ or $1 \mathrm{~b}$ and collinear usage of exons 2-6 (figure 5 a and c; Molitor, 2002). The conceptual translation of the $2695 \mathrm{bp}$ and $2542 \mathrm{bp}$ transcripts resulted in two hypothetical protein sequences of 546 aa and 339 aa 
a)
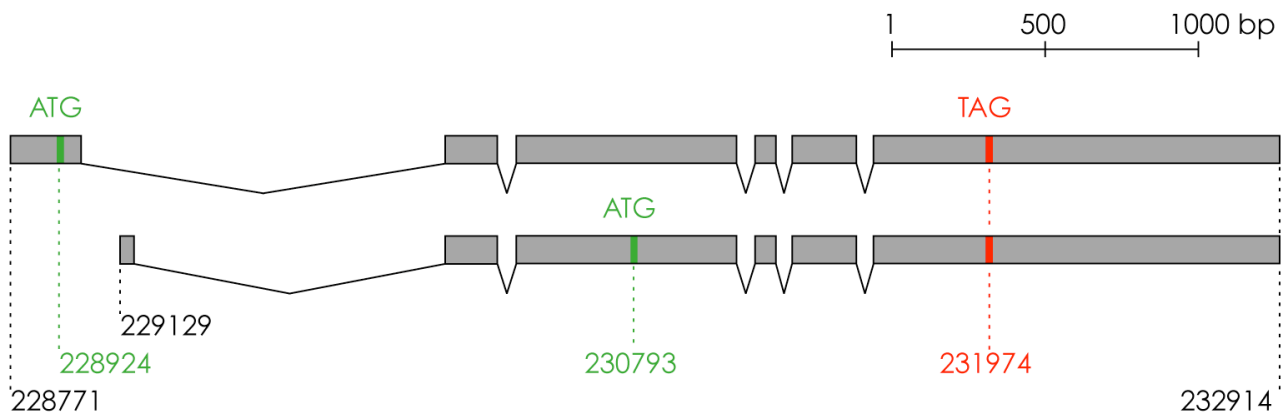

b)

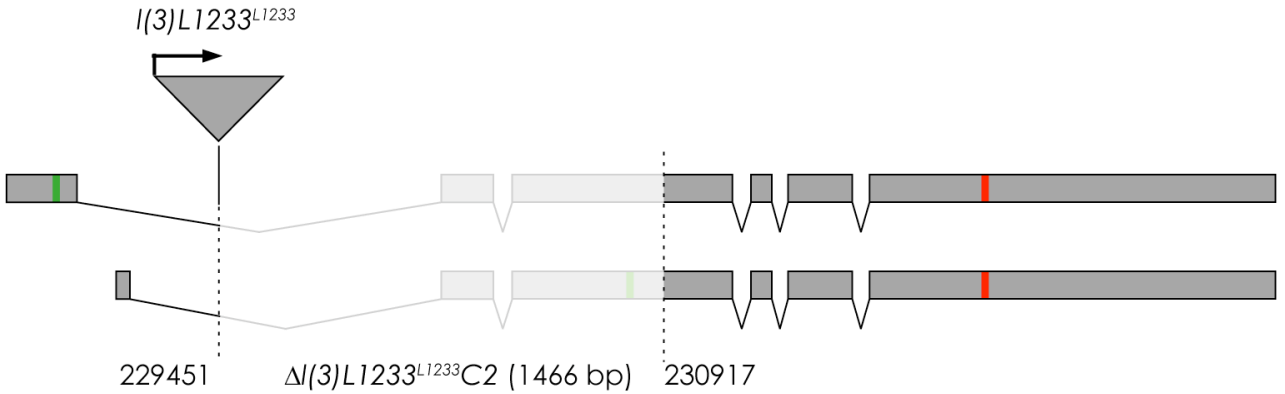

c)

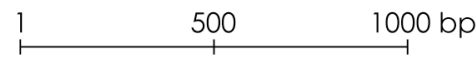

:Exon 1a: Exon $2:$ Exon $3:$ Exon $4:$ Exon 5

Exon 6

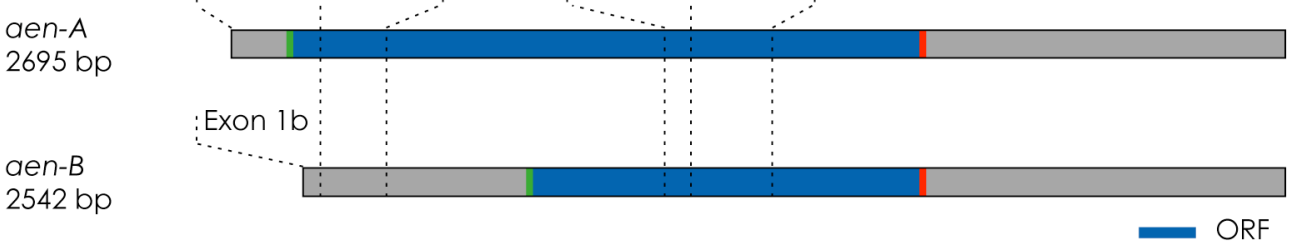

d)

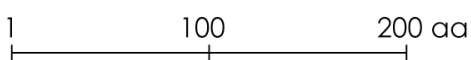

Aen-A

$546 \mathrm{aa}$

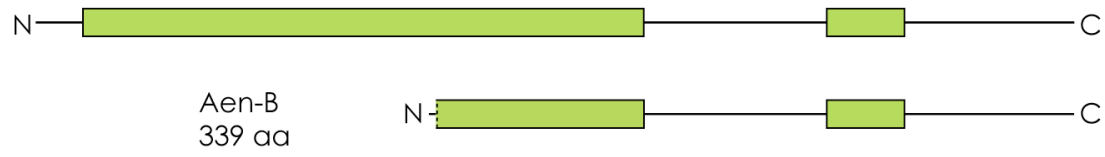

Figure 5: The aeneas gene and its products. a) aeneas (aen) locus on chromosome arm 3R; cytogenetic band 82A6; total length: $4143 \mathrm{bp}$; numbers indicate absolute base positions on the chromosome. b) aen mutations; position of the P-element insertion $P\{\mid a C W\} \mid(3) L 1233^{L 1233}$ in aen' mutants; coverage of the deletion $\Delta /(3) L 1233^{L 1233} C 2$ in aen ${ }^{2}$ mutants. C) aen transcripts; position of splice sites and the positions of the ORFs (blue) of both transcript variants. d) Aen protein variants; the conserved regions, inferred from BLAST search analysis, are depicted in green.

respectively (figure $5 d$ ). In the course of this study these variants will be termed AenA and AenB. Sequence comparison of the AenA protein sequence with sequences obtained from the computational database showed, that Aen belongs to a conserved group of proteins (Molitor, 2002). 
To analyze the aen gene in mutant condition, flies of the line aen', which contains a transposable P-element I(3)L1233R80 in the first intron of both transcripts, were analyzed. It was found that no apparent muscle phenotypes occur in embryos originating from aen' parents. However, the embryos exhibit defects in PGC migration (Molitor, 2002). For further analysis of the aen gene, a null mutant allele was generated by remobilization of the /(3)L 1233L1233 P-element (Robertson et al., 1988), which led to removal of genomic sequence by imprecise excision in one of the lines generated. The line aen ${ }^{2}$ was established, which contains the deletion $\mathrm{Al}(3) \mathrm{L} 1233^{L 1233} \mathrm{C} 2$ covering $1466 \mathrm{bp}$. This deletion results in the removal of the complete second and about half of the third exon of aen (Molitor, 2002) (figure 5 b). Primary analysis of the P-insertion and the deletion mutant alone or in trans to the deficiency $D f(3 R)(X M 3)$ removing the entire genomic region showed that in all cases premature transmigration of PGCs through the newly formed epithelium of the PMG occurs (Molitor, 2002).

Analysis of aen transcription by a developmental Northern Blot showed that the gene is expressed weakly throughout development but that there is strong maternal expression (Molitor, 2002). During early embryogenesis, at stages 1 to 5, high levels of aen transcripts are detectable. Visualization of the aen transcripts in whole mount in situ stainings of embryos showed that aen transcripts are ubiquitously present during stages 1 to 4 and with stage 5 are reduced in the blastoderm with exception of the PGCs and the PMG epithelium (Molitor, 2002). At stage 9 to 10, aen transcript levels were reduced in the PGCs and instead became detectable in the domains of the invaginating midgut and, from stage 11 to 12 , in the visceral mesoderm (Molitor, 2002.) 
The objective of my work was to characterize the biological and molecular function of the Aen protein in order to understand the mechanisms underlying the temporal and spatial control of invasive PGC migration.

For this purpose, a detailed analysis of the PGC mismigration phenotype in aen mutants was performed. The abundance of Aen protein variants in the course of oogenesis and embryogenesis was examined. Factors interacting with the Aen protein were identified and the biological relevance of the interactions found was characterized both functionally in genetic interaction experiments as well as on the molecular level in a detailed biochemical analysis. 


\section{Materials and methods}

\subsection{Molecular Biology}

\subsubsection{Small scale plasmid-DNA preparation}

All chemicals if not specified otherwise were obtained from SigmaAldrich.

Small amounts of plasmid DNA were isolated from 1,5 ml bacterial culture according to the LiCl-Boiling method (Sambrook, 1989) or using the QuiaQuick mini prep Kit (Qiagen) according to the protocol supplied by the manufacturer. The dried DNA pellet was redissolved in $40 \mu \mathrm{ITE}$ buffer at $60^{\circ} \mathrm{C}$.

\subsubsection{Medium scale plasmid DNA preparation}

For midi preparations of Plasmid DNA, a $150 \mathrm{ml}$ bacterial culture was grown overnight. The DNA preparations were then carried out with the Qiagen Plasmid Midi Kit (Qiagen) according to the protocol supplied by the manufacturer. The DNA pellet was redissolved in $100 \mu \mathrm{l} \mathrm{TE} \mathrm{buffer}$ at $60^{\circ} \mathrm{C}$.

\subsubsection{Large scale plasmid DNA preparation}

For maxi preparations of Plasmid DNA, a $300 \mathrm{ml}$ culture was grown overnight. The DNA preparations were then carried out with the Qiagen EndoFree Maxi Kit (Qiagen) according to the protocol supplied by the manufacturer. The DNA pellet was redissolved in $100 \mu \mathrm{l}$ EndoFree $\mathrm{EB}$ at $60^{\circ} \mathrm{C}$. 


\subsubsection{Extraction of genomic DNA from flies}

For extraction of genomic DNA from flies (adapted from Gloor et al., 1993), a single fly was placed in a $1.5 \mathrm{ml}$ reaction tube and paralyzed by cold. The fly was squished with a pipet tip and suspended in $50 \mu$ of squishing buffer $110 \mathrm{mM}$ Tris-Cl pH 8.2, 1 mM EDTA, 25 mM NaCl, 200 $\mu \mathrm{g} / \mathrm{ml}$ Proteinase K (Sigma)). After $30 \mathrm{~min}$ incubation at $37^{\circ} \mathrm{C}$, the Proteinase K was heat-inactivated by incubation for 2 min at $95^{\circ}$.

\subsubsection{Determination of DNA concentration}

For the determination of the DNA concentration in aqueous solute, a Genesys 6 Spectrophotometer (Thermo Spectronic) was used for absorption measurement at $260 \mathrm{~nm}$ wavelength.

\subsubsection{Sequencing of DNA}

DNA-sequence analysis was carried out MWG-Biotech AG or by Gordon Dowe (MPI biophysical chemistry) using the Prism ready reaction kit $(A B I)$ together with the automated sequencer $A B I 377 / 96$ $(A B I)$. The samples were diluted to $100 \mathrm{ng} / \mu \mathrm{l}$. $500 \mathrm{ng}$ of template, 6pmol of primer and $2.5 \mu \mathrm{l}$ of kit solution were combined for the sequencing reaction.

\subsubsection{Sequence analysis and Primer design}

For the alignment and analysis of DNA sequences, the software Sequencher (Gene Codes Cooperation) was utilized. For the primerdesign the software OLIGOß 4.06 Primer Analysis Software (National Biosciences) was used. Reference sequences were retrieved from NCBI (http://www.ncbi.nlm.nih.gov; Altschul et al., 1990) and Flybase (http://flybase.bio.indiana.edu; Flybase, 2003). 
2.1.8 Sequence IDs of the sequences included in the phylogenetic tree of AenA

The AenA full-length protein sequence was retrieved from Flybase (http://flybase.bio.indiana.edu/). Sequences Conserved between 11 Drosophila species were identified by BLAT search (http://genome.ucsc.edu/index.html) and the software-tool Evoprinter (http://evoprinter.ninds.nih.gov). Homologs of the N-terminal conserved domain covering 294 aa from Dm-AenA residue 25 to 318 were identified by BLAST search (http://www.ncbi.nlm.nih.gov/BLAST/; Altschul et al., 1990) using the blastp algorithm. The output was restricted with a difference cutoff of 0.7. The phylogenetic tree was generated using the BLAST Tree Widget (http://www.ncbi.nlm.nih.gov/BLAST/).

NP_649466 (Drosophila melanogaster); XP_001359602 (Drosophila pseudoobscura); EAT46680 (Aedes aegypti); XP_395864 (Apis mellifera); XP_966481 (Tribolium castaneum); XP_310457 (Anopheles gambiae); NP_00103950 (Bos Taurus); XP_851637 (Canis familiaris); NP_001038564 (Danio rerio); XP_001085714 (Macaca mulatta); XP_414185 (Gallus gallus); BAB14383 (Homo sapiens); XP_511153 (Pan troglodytes); XP_001376232 (Monodelphis domestica); CAF99528 (Tetraodon nigroviridis); BAE24631 (Mus musculus); CAH89819 (Pongo pygmaeus); XP_001195086 (Strongylocentrotus purpuratus); AAH94085 (Xenopus laevis)

\subsubsection{PCR reactions and conditions}

For analytical PCR, the HotStar Taq Mastermix kit (Qiagen) was used according to the instruction manual. For amplification of sequences below $3 \mathrm{~kb}$, the PfuTurbo DNA polymerase (Stratagene) was utilized. For Long range PCR with a designated product above $3 \mathrm{~kb}$, the Expand Long Template PCR System (Roche) or the La Taq Kit (TAKARA) 
were used according to the instruction manual. The annealing temperatures of were chosen $5^{\circ} \mathrm{C}$ below the mean annealing temperature of the designated Primers. All PCRs were carried out in a Gene Amp 9700 PCR cycler (Applied Biosystems).

\subsubsection{DNA digest with restriction endonucleases}

Restriction digests were carried out according to the methods described in Sambrook et al. (1989). The software EnzymeX (The Netherlands Cancer Institute) was used for the determination of buffer conditions in parallel digests. Generally, $1 \mu \mathrm{g}$ DNA was digested in $50 \mu \mathrm{l}$ total volume including buffer and restriction endonucleases (Fermentas, New England Biolabs) at the concentrations indicated in the respective instruction manuals. The restriction digest was carried out at $30-37^{\circ} \mathrm{C}$ for $2 \mathrm{~h}$ and subsequently stopped by heat inactivation of the restriction endonucleases at $60^{\circ} \mathrm{C}$ for $10 \mathrm{~min}$. For buffer exchange in sequential restriction digests, the DNA samples were instead of heat inactivation purified using the QiaSpin Nucleotide Removal Kit (Qiagen) and eluted in 40-45 $\mu \mathrm{l} \mathrm{H}_{2} \mathrm{O}$.

\subsubsection{Agarose-gel electrophoresis}

For analysis or purification of restriction fragments, the reactions were subjected to agarose-gel electrophoresis on $0.8-1.5 \%$ gels in $0.5 \times$ TBE buffer (Sambrook et al. 1989) including Ethidiumbromide (Roth). The stained DNA fragments visualized next to GeneRuler DNA Ladder Mix (Fermentas) with an UV-transilluminator (Raytest) at $366 \mathrm{~nm}$.

\subsubsection{Gel extraction of DNA}

DNA fragments were isolated from agarose gel with the QiaSpin Gel Extraction Kit (Qiagen) according to the protocol supplied by the manufacturer. 


\subsubsection{DNA ligation}

For ligation (modified from Sambrook et al. 1989), digested plasmids and inserts with compatible ends were mixed in a molar ratio of 4:1 and included in $10 \mu$ reaction volume with T4 DNA Ligase (Fermentas) according to the manufacturers manual. Ligation was performed overnight at $14^{\circ} \mathrm{C}$.

\subsubsection{Gateway recombination}

For gateway recombination, Entry-vectors containing the designated insert inside an rfa-recombination cassette were mixed with Destination vectors containing promoter region and fusion-tags as well as selective traits. The recombination was carried out with the Gateway recombination kit (Invitrogen) according to the manufacturers instruction.

\subsubsection{Heatshock transformation of bacterial strains}

For transformation, 100-200 ng of DNA were mixed with $50 \mu \mathrm{l}$ chemocompetent E. coli (protocol adapted from Sambrook et al. 1989). After 30-45 min preincubation on ice, the cells were subjected to a heatshock by $30 \mathrm{~s}$ incubation at $42^{\circ} \mathrm{C}$ and transferred back on ice. After $1 \mathrm{~h}$ recovery in $300 \mu \mathrm{LB}, 50 \mu \mathrm{l}$ bacterial suspension were plated on LB-Agar plates containing selective antibiotics.

\subsubsection{Phenolization of plasmid DNA}

For injection of constructs into fly embryos, the DNA needs to be of high purity. Any contamination with proteins and potentially toxic agents has to be avoided. Therefore, the acquired constructs were further processed after Midi Preparation. A phenolization was carried out. The whole DNA yielded by the Midi preparation was resolved in $300 \mu$ final volume of $\mathrm{H}_{2} \mathrm{O}$ and transferred into a Phase Lock Gel 
reaction vial (Eppendorf-Netheler-Hinz GmBH). $300 \mu$ Phenol were added and the liquids were suspended by vortexing thoroughly. For phase separation, the suspension was centrifuged for 2' at $12 \mathrm{krpm}$. The watery (upper) phase was transferred into a new a Phase Save reaction vial and $300 \mu \mathrm{l}$ Phenol/Chloroform were added. The liquids were suspended by vortexing thoroughly and centrifuged. The watery phase was transferred into a new vial and twice washed with $300 \mu \mathrm{l}$ chloroform as described above. This way, all Phenol was removed from the sample. Then the DNA was precipitated adding $30 \mu \mathrm{l}$ of $3 \mathrm{M}$ Sodium-Acetate and $750 \mu$ Ethanol (100\%, p.A.). For pelleting the DNA was centrifuged for $15^{\prime}$ at $12 \mathrm{krpm}$. The supernatant was discarded. Then the DNA was rinsed with $500 \mu$ Ethanol (70\%) to remove residual salt. The DNA was again centrifuged for 5' at $12 \mathrm{krpm}$ and the supernatant was discarded. The DNA pellet was dried in the SpeedVac for 5' and resolved in $20 \mu \mathrm{l} \mathrm{H}_{2} \mathrm{O}$ LiChrosolv; Merck).

\subsubsection{Preparation of injection mixture from Plasmid DNA}

For injection, the DNA was combined with a helper construct that expresses the P-Element-Transposase that enables insertion of the construct into the fly genome. For this purpose, $12 \mu \mathrm{g}$ of the DNA and 4 $\mu \mathrm{g}$ helper construct were diluted in $200 \mu$ final volume of $\mathrm{H} 2 \mathrm{O}$ and precipitated as described in paragraph 2.1.9. The DNA pellet was then resolved in $20 \mu \mathrm{l} \mathrm{H}_{2} \mathrm{O}$ (HPLC grade) and the concentration was spectrophotometrically determined. For injection (see paragraph 2.2.1), the DNA concentration was adjusted to $400 \mathrm{ng} / \mu \mathrm{l}$.

\subsection{Genetics}

\subsubsection{Germ line transformation}

For the germ line transformation of fly embryos with P-Element-Vectors (Rubin and Spradling, 1982), the DNA was prepared as described in 
section 1.1.16. The DNA mixture was then injected into early the pole plasm of pre-blastoderm embryos of the flystock $w^{s n 3}$. The flies developing after this procedure were crossed back against wsn3. Transgenic flies were selected from the F2 generation by the expression of the marker gene white indicating the integration of the P-element-plasmid into the genome. Injections of into fly embryos were carried out by Iris Plischke and Ursula Jahns-Meyer (MPI biophysical chemistry).

\subsubsection{Culturing of flies}

Flies were cultured according to the methods described by Ashburner (Ashburner, 1989). Generally, flystrains were grown at $18-22^{\circ} \mathrm{C}$ on standard Cornmeal/Agar substrate.

\subsubsection{Crosses for the genetic interaction analysis between aen and slam}

For the analysis of the slam single mutants, the offspring of $\mathrm{w}^{*}$;wdo ${ }^{20.84} \mathrm{~m} /$ Cyo,ftz-lacz heterozygous slam ${ }^{l}$ mutant females and Df(2L)ED369, $P\left\{w^{+m w}\right.$.Scer \FRT.hs3 $=3$ '. RS5+3.3' $\}$ ED369/Cyo, hb-lacz heterozygous males was analyzed. While the remaining half of maternal contribution is equally present in all embryos originating from this cross, four genotypes (denoted as slam\#;+ in figure 17 and 19) occur in equal ratios:

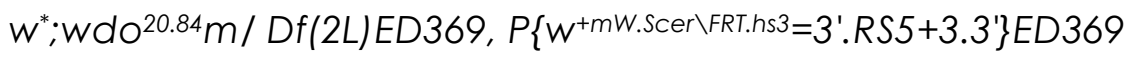

$w^{*} ; w d o^{20.84} \mathrm{~m} /$ Cyo, hb-lacz

Df(2L)ED369, P\{w'mw.Scer \FRT.hs3=3'.RS5+3.3' $\}$ ED369/Cyo, ftz-lacZ

Cyo,ftz-lacz/Cyo,hb-lacz 
For the analysis of the slam-aen double mutants, following the same scheme, the offspring of $w^{*} ; w^{2} o^{20.84} \mathrm{~m} / \mathrm{Cyo}, \mathrm{ftz}-\mathrm{lacz} ; \mathrm{aen}^{2}$ heterozygous slam' mutant females, bearing the homozygous aen² mutation, and Df(2L)ED369, P\{w'mw.Scer\FRT.hs3=3'.RS5+3.3'\}ED369/Cyo,ftz-lacZ; aen ${ }^{2}$ males was analyzed, resulting in homozygous aen ${ }^{2}$ mutant offspring bearing half of the slam maternal contribution. The genotypes generated in this cross (denoted as slam\#; aen² in figure 17 and 19) are:

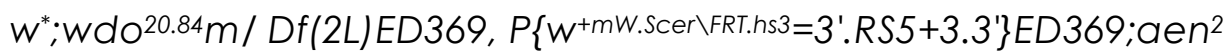

$\mathrm{w}^{*}$;wdo ${ }^{20.84} \mathrm{~m} /$ Cyo,ftz-lacz;aen²

Df(2L)ED369, P\{w'mw.Scer\FRT.hs3=3'.RS5+3.3'\}ED369/Cyo,ftz-lacZ; aen ${ }^{2}$

Cyo,ftz-lacz/Cyo,ftz-lacz;aen²

\subsection{Protein biochemistry}

\subsubsection{Generation of the Aen antibody}

The anti-Aen antibody was generated by Eurogentec by immunization of rabbits with two synthesized peptides.

Peptide I : C+ETAQKRTPRRKGRFV

Peptide II: C+DVGGARIEAVDTTTV

The sera received from Eurogentec were subjected to affinity purification using both synthesized antigens on $\mathrm{CNBr}$-activated Sepharose 4B (Amersham) according to the manufacturers instruction.

\subsubsection{Collection of embryos and dissection of ovaries}

For egg laying, flies were kept on apple agar plates supplemented with yeast (adapted from Rothwell and Sullivan, 2000). Embryos for Western blotting were collected in intervals from 0 to $3 \mathrm{~h}$, from 3 to 10 $\mathrm{h}$ and from 10 to $22 \mathrm{~h}$. Embryos were scraped of the agar surface and 
transferred into sieves. For removal of the chorion, the embryos were incubated in a 50 \% solution of custom detergent for 3 minutes at RT. The detergent solution was thoroughly washed off with isotonic embryo wash solution $(0,8 \% \mathrm{NaCl}, 0,1 \%$ Triton X-100).

Ovaries were dissected from approximately 4 days old adult females in isotonic embryo wash solution (modified from Heinrich et al., 2000; Rothwell and Sullivan, 2000). All samples were stored at $-20^{\circ} \mathrm{C}$.

\subsubsection{Protein extraction from embryos and ovaries}

About $20 \mu$ l of embryos, larvae, pupae or ovaries were transferred into a reaction vial and covered with $100 \mu$ I RIPA buffer $(20 \mathrm{mM}$ Tris pH 7,5; 138 mM NaCl; 0,1 \% SDS; 0,5 \% DOC; 1 \% Triton X-100; 2 mM EDTA; $10 \%$ Glycerol) (modified from Harrison et al., 1998). After three times freezing at $-80^{\circ} \mathrm{C}$ and thawing at $55^{\circ} \mathrm{C}$, the samples were grinded twice with a potter. The debris was spinned down (2', 5 krpm) and the supernatant was transferred into a new tube.

The protein concentrations were measured against a BSA standard with the BCA Protein Assay Kit (Pierce) in a microtiter plate reader.

Alternatively, the samples were directly covered with 1:5 (V/V) SDS loading dye (Sambrook et al., 1989), grinded with a potter and heated to $95^{\circ} \mathrm{C}$ for $5^{\prime}$.

\subsubsection{SDS-PAGE}

SDS polyacrylamide gelelectrophoresis was performed using the Mini Protean II system (Bio-Rad). Gels and buffers were prepared according to standard procedures (Sambrook et al., 1989). PageRuler Prestained Protein Molecular Weight Marker (Fermentas) was used for Protein size estimation. 


\subsubsection{Western blot}

The Western blots were carried out according to standard procedures (Sambrook et al., 1989) using the Mini Protean II system (Bio-Rad, Munich, Germany). For blotting, Nitrocellulose membranes (Schleicher \& Schuell) or PVDF membranes (Immobilon-P) were used. Signals from HRP coupled secondary antibodies were generated using the SuperSignal West Pico Chemiluminiscent Substrate Kit (Pierce). Detection of HRP signals was carried out in an Intelligent Dark Chamber II (Fujifilm Europe $\mathrm{GmbH}$ ) or by exposition of X-Ray films (Kodak BioMax XAR Film, Kodak). After exposure, films were developed in a developing machine (X-Ray Automatic Processor QX60, Konica).

\subsubsection{Protein expression and purification}

For bacterial expression in E. coli BL21 (DE3) (adapted from Sambrook et al., 1989), precultures were grown in LB in $50 \mathrm{ml}$ Volume at $37^{\circ} \mathrm{C}$ over night. Expression cultures were inoculated at a ratio of 1: 20 from preculture to final Volume in LB. Expression cultures were grown until their optical density (OD) reached $O D=0,5$ at $600 \mathrm{~nm}$ wavelength. ODmeasurements were done with a Genesys 6 Spectrophotometer (Thermo Spectronic). For induction of protein expression, aqueous IPTG (Sigma) solution was added to a final concentration of 0,4 $\mathrm{mM}$. The cultures were incubated at $30^{\circ} \mathrm{C}$ until the $\mathrm{OD}$ reached a value of $O D=1,0$. Bacteria were harvested by centrifugation in a RC-5B Sorvall Refrigerated Superspeed Centrifuge (Sorvall) at $4 \mathrm{krpm}$ and $4^{\circ} \mathrm{C}$ for 15 min. The bacterial pellets were resuspended in ice-cold PBS $1130 \mathrm{mM}$ $\mathrm{NaCl}, 7 \mathrm{mM} \mathrm{Na} 2 \mathrm{HPO}_{4}, 3 \mathrm{mM} \mathrm{NaH} \mathrm{PO}_{4}$ ) and again pelleted by centrifugation at $4 \mathrm{krpm}$ and $4^{\circ} \mathrm{C}$ for $10 \mathrm{~min}$. Then the pellets were resuspended in $20 \mathrm{ml}$ HEMG-1 buffer per 11 culture volume $(25 \mathrm{mM}$ Hepes- $\mathrm{KOH}$ ph 7,6, 0,1 mM EDTA, $12,5 \mathrm{mM} \mathrm{MgCl}_{2}, 0,5 \mathrm{M} \mathrm{NaCl}, 0,1 \%$ NP40 (IGEPAL CA-630 (Sigma), 10\% Glycerol), Complete Roche 
Protease Inhibitor mix (Roche) and Lysozyme (Biomol) was added to $10 \mathrm{mg} / \mathrm{l}$ final concentration. After $30 \mathrm{~min}$ incubation at $4^{\circ} \mathrm{C}$, the bacterial lysates where subjected to 2 freeze-thaw cycles and homogenized by sonification. The debris was pelleted from the lysates by centrifugation at $15 \mathrm{krpm}$ and $4^{\circ} \mathrm{C}$ for $20 \mathrm{~min}$. For affinity purification, 50 \% slurry of Glutathione sepharose beads (Amersham Biosciences) was preconditioned by rinsing with HEMG-1 buffer (without protease inhibitors). $20 \mathrm{ml}$ of cleared culture supernatant were added to $3 \mathrm{ml}$ bed volume of $50 \%$ Glutathione sepharose beads and rotated at $4^{\circ} \mathrm{C}$ for $2 \mathrm{~h}$ to allow binding. Subsequently, the resin was trice washed with HEMG-1, HEMG-2 $(25 \mathrm{mM}$ Hepes-KOH ph 7,6, 0,1 mM EDTA, 12,5mM MgCl $2,0,1 \mathrm{M} \mathrm{NaCl}$, 0,1 \% NP40 (IGEPAL CA-630, Sigma), $10 \%$ Glycerol) and HEMG-3 (25 mM Hepes-KOH ph 7,6, 0,1 mM EDTA, $12,5 \mathrm{mM} \mathrm{MgCl}_{2}, 0,1 \mathrm{M} \mathrm{NaCl}, 0,01 \%$ NP40 (IGEPAL CA-630, Sigma), 10\% Glycerol) respectively. The washed resin was mixed 1:1 with HEMG-3 including Protease inhibitors. For storage, the resin was aliquoted to 20 $\mu \mathrm{l}$ bedvolume (+20 $\mu$ l buffer), frozen in liquid $\mathrm{N}_{2}$ and stored at $-80^{\circ} \mathrm{C}$.

\subsubsection{In vitro translation}

For in vitro transcription/translation, the TNT Coupled Reticulocyte Lysate System (Promega) was used according to the provided protocol to produce radiolabelled (35S) proteins in $50 \mu$ reactions. As a radioactive label, the Redivue ProMix (35S) (Amersham Bioscience) was applied. Translation efficiency was assayed with a Luciferase control reaction (supplied by the manufacturer).

\subsubsection{GST-Pulldown}

For GST-pulldown, the in vitro translation reactions were diluted to 260 $\mu$ l total volume with HEMG-1 buffer (see section 2.3.8) including protease inhibitors and centrifuged at $12 \mathrm{krpm}$ and $4^{\circ} \mathrm{C}$ for $15 \mathrm{~min}$ (protocol adapted from Blanke and Jäckle, 2006). 40 ul His6-Gst-AenA 
or His6-Gst bound Glutathione sepharose (50\% slurry) was mixed with $50 \mu \mathrm{l}$ of diluted translation product $(1: 5,2)$. Additionally $1 \mu \mathrm{l}$ of Luciferase control reaction (undiluted) was added to serve as an internal negative binding control. To allow binding of in vitro translated proteins, the resin was rotated at $4^{\circ} \mathrm{C}$ for $2 \mathrm{~h}$. After 5 washing steps of 10 min at RT with 10 bed volumes of HEMG-1 buffer, $15 \mu$ l of resin was mixed with $30 \mu \mathrm{l}$ SDS loading dye (Sambrook et al., 1989). After denaturing by incubation for $5 \mathrm{~min}$ at $95^{\circ} \mathrm{C}, 10 \mu \mathrm{l}$ of each sample were loaded onto 12 \% SDS-PAGE gels next to PageRuler Prestained Protein Molecular Weight Marker (Fermentas). The gels were dried overnight and exposed to Kodak BioMax XAR X-Ray films (Kodak) for 18-24 hours at $-80^{\circ} \mathrm{C}$.

\subsubsection{Cell culture conditions}

Kc167-cells (Cherbas et al., 1977) originating from Drosophila, were grown in $75 \mathrm{~cm}^{2}$ flasks (BD Biosciences) containing $10 \mathrm{ml}$ Schneider's Drosophila medium (Gibco Invitrogen) which was supplemented with 10\% fetal calf serum (Sigma-Aldrich) and penicillin/streptomycin (0.1 $\mathrm{mg} / \mathrm{ml}$, PAA Laboratories) at $25^{\circ} \mathrm{C}$.

\subsubsection{Expression in cell culture}

For expression of fusion proteins in cell culture, cells were seeded at a density of $5 \times 10^{5}$ per well of a 6 -well plate and allowed to settle for 1$2 \mathrm{~h}$ at $5^{\circ} \mathrm{C}$. Transfection was carried out using the Effectene Transfection Reagent (Qiagen) according to the supplied manual.

\subsubsection{Co-IP}

For Co-IP, transfected cells were grown at $25^{\circ} \mathrm{C}$ for 4 days until they reached confluency. The cells were harvested by thorough rinsing and transferred into $15 \mathrm{ml}$ conical bottom tubes (Sarstedt). After 
sedimenting for 2 min at $4 \mathrm{krpm}$ and $4^{\circ} \mathrm{C}$, the cell pellet was resuspended in $10 \mathrm{ml}$ Rinse-buffer (40 mM Tris pH 7,5, $1 \mathrm{mM}$ EGTA, 0, 15 $\mathrm{M} \mathrm{NaCl}$ ) and again sedimented. Then, the cells were resuspended in $400 \mu$ l RIPA-buffer $(50 \mathrm{mM}$ Tris ph 7,5, $50 \mathrm{mM} \mathrm{NaCl}, 1 \mathrm{mM}$ DTT, 0,1 \% NP40 (IGEPAL CA-630, Sigma), 10 \% Glycerol, Complete Roche Protease Inhibitor mix (Roche)) and disrupted by 3 freeze-thaw cycles using liquid $\mathrm{N}_{2}$. After pelleting of debris for $15 \mathrm{~min}$ at $13 \mathrm{krpm}$ and $4^{\circ} \mathrm{C}$, the supernatants were frozen in liquid $\mathrm{N}_{2}$ and stored at $-80^{\circ} \mathrm{C}$.

For co-immunoprecipitation, $1 \mathrm{ml}$ AffiPrep ProteinA Support resin (BioRad) was equilibrated by washing with PBS (protocol adapted from Harlow and Lane, 1999). For binding, $\mathrm{NaCl}$ was added to $4 \mathrm{ml}$ anti-Myc (lowa-Hybridoma Bank) cell supernatant to a final concentration of $3 \mathrm{M}$. The serum was added to the resin and rotated overnight at $4^{\circ} \mathrm{C}$. Afterwards, the resin was $3 \times$ rinsed and $2 \times$ washed for 20 min with RIPA-buffer. The culture cell extracts were thawed on ice. $30 \mu \mathrm{l}$ were saved as an input control, mixed with $30 \mu \mathrm{l}$ of protein loading dye (Sambrook et al., 1989), denatured at $95^{\circ} \mathrm{C}$ for 5 min and stored at $-20^{\circ} \mathrm{C}$. The residual $370 \mu \mathrm{l}$ of extract were mixed with $30 \mu \mathrm{l}$ of preconditioned resin and incubated for $24 \mathrm{~h}$ at $4^{\circ} \mathrm{C}$. The supernatant was removed and the resin was twice rinsed and washed $4 \times 30$ min with RIPA. Then, $30 \mu \mathrm{l}$ resin and $15 \mu \mathrm{l}$ RIPA were mixed with $30 \mu \mathrm{l}$ of Protein loading dye (Sambrook et al., 1989) and denatured at $95^{\circ} \mathrm{C}$ for $5 \mathrm{~min} .10 \mu \mathrm{l}$ of each sample were loaded onto $8 \%$ SDS-PAGE gels next to PageRuler Prestained Protein Molecular Weight Marker (Fermentas) and subjected to Western Blot (see section 2.3.5).

\subsection{Histology and Embryology}

\subsubsection{Formaldehyde fixation of Drosophila embryos}

For fixation, embryos were collected and dechorionated as described in section 2.3.1. Collection intervals of $2-4 \mathrm{~h}$ embryonic age were 
chosen. For stainings using the anti-Vasa antibody, the embryos then were fixated on the shaker in $8 \mathrm{ml}$ Heptane, $0,5 \mathrm{ml}$ Formaldehyde (37\%) and Fixation buffer (100 mM HEPES; 2 mM MgSO $; 1$ mM EGTA, pH 6,9) for 20' (modified from Rothwell and Sullivan, 2000). The aqueous phase was removed. The embryos were devitellinized by addition of methanol and vigorous shaking. The Heptane- and interphase were removed and the embryos were three times washed with methanol. For storage, the embryos in methanol were kept at $-20^{\circ} \mathrm{C}$.

\subsubsection{Paraformaldehyde fixation of embryos}

For stainings of epithelial morphology, embryos were fixated on the shaker in 5ml Heptane, in 4ml PEM-buffer (100 mM PIPES, 5 mM EGTA, 2 $\mathrm{mM} \mathrm{MgCl} 2, \mathrm{pH} 6.8,0.2 \%$ Triton X-100 (TX-100)) including 5\% PFA and 1 $\mathrm{ml}$ Picric acid (Sigma; 13\% aqueous solution) for 20' (modified from Kreitmeier et al., 1995). The aqueous phase was removed. The embryos were devitellinized by addition of methanol and vigorous shaking. The Heptane- and interphase were removed and the embryos were three times washed with methanol. For storage, the embryos in methanol were kept at $-20^{\circ} \mathrm{C}$.

For stainings using fluorescently labeled phalloidin, fixation was carried out as described above replacing Methanol with $80 \%$ ethanol.

\subsubsection{Dissection and fixation of ovaries}

Ovaries were dissected as described in section 2.3 .2 and fixated in $5 \mathrm{ml}$ PEM-PFA (100 mM PIPES, 5 mM EGTA, 2 mM MgCl2, pH 6.8, 0.2\% Triton $\mathrm{X}-100$ (TX-100)) including 4\% PFA for 20 min. Fixation was stopped by $2 x$ rinsing with $\mathrm{PBT}$ (PBS, 0,1\% Tween20). For storage, ovaries were dehydrated stepwise in ethanol and stored at $-20^{\circ} \mathrm{C}$. 
2.4.4 Generation of transcript specific probes for RNA in situ detection

For labeling of RNA-probes, the RNA- Labeling and Detection Kit (Roche) was used according to the manufacturers manual. After labeling, $1 \mu$ l DNasel (RNase-free) was added to the samples followed by $15 \mathrm{~min}$ incubation at $37^{\circ} \mathrm{C}$. The labeled probe was purified using the RNeasy Mini Kit (QIAGEN), following the instruction manual.

\subsubsection{RNA in situ detection in fixated embryos and ovaries}

For RNA in situ staining (modified from Lehmann and Tautz, 1994) ovaries were dissected and fixated as described in section 2.4.3. The ovaries were washed in PBT $3 \times 5$ min and prefixated for 20 min in PBS including $4 \%$ formaldehyd. After further washing steps with PBT, the ovaries were transferred into the hybridization solution in a stepwise manner. Equilibration steps included $10 \mathrm{~min}$ in (1:1) PBT:Hype-B (50\% Formamid; $5 \times$ SSC; 0,1 \% Tween20), in 10 min Hybe-B and 10 min in (1:1) Hybe-B:HybeA (50\% formamide, 5x SSC, $0.2 \mathrm{mg} / \mathrm{ml}$ sonicated salmon testis DNA, $0.1 \mathrm{mg} / \mathrm{ml}$ tRNA, $50 \mu \mathrm{g} / \mathrm{ml}$ heparin).

The ovaries were prehybridized in Hybe-A for $1 \mathrm{~h}$ at $65^{\circ} \mathrm{C}$. Then, the buffer was replaced by $30 \mu \mathrm{l}$ Hybe-A including $2 \mu \mathrm{l}$ DIG-labeled RNAprobe. The ovaries were incubated over night at $65^{\circ} \mathrm{C}$ in a waterbath. After hybridization, the ovaries were washed $10 \mathrm{~min}$ at $65^{\circ} \mathrm{C}$ with prewarmed Hybe-A, $2 \times 15$ min at $65^{\circ} \mathrm{C}$ with prewarmed Hype-B, 15 min at RT in PBT:Hybe-B (1:1) and $4 \times 15$ min at RT in PBT. For detection of the DIG-labeled RNA-Probes, the ovaries were incubated with an anti-Dig AP-conjugated antibody 1:2000, Roche Diagnostics, Mannheim) for 1 h at $37^{\circ} \mathrm{C}$. After $6 \times 15$ min washing in PBT at RT the ovaries were transferred into AP-Puffer (20 mM Tris pH 9,5; $100 \mathrm{mM}$ $\mathrm{NaCl}$; $50 \mathrm{mM} \mathrm{MgCl}$ ) and washed $3 \times 5 \mathrm{~min}$. For the development of the staining, 4,5 I NBT and 3,5 I BCIP were added into $1 \mathrm{ml}$ AP-Puffer. The staining was stopped by $3 \times 5$ min and $1 \times 20$ min washing in PBT. 
The stained ovaries were dehydrated in an ethanol dilution series $130 \%$, $50 \%, 70 \%, 90 \%$ and $2 \times 100 \%$ ), mounted on a slide in Canada Balsam (Sigma-Aldrich) and covered with a coverslip.

\subsubsection{Antibody staining of Drosophila embryos}

About $20 \mu \mathrm{l}$ of fixated embryos were rehydrated by three times 5' washing with PBT including $5 \%$ goat serum (protocol adapted from Rothwell and Sullivan, 2000). Incubation with the primary Antibody was done at $4^{\circ} \mathrm{C}$ over night. The primary antibody was then removed by twice washing with PBT including $2 \%$ of goat serum. Then, either fluorescence coupled or biotinylated secondary antibodies (Molecular Probes) were applied, diluted in $380 \mu \mathrm{l}$ PBT including $10 \mu \mathrm{l}$ Serum, and incubated on the embryos for $2 \mathrm{~h}$. All following steps were carried out at RT.

For detection of the fluorescence-coupled antibodies, the embryos after incubation were washed $6 \times 10^{\prime}$ in PBT, $2 \times 10^{\prime}$ in PBT and $2 \times 5^{\prime}$ in PBS. The embryos were allowed to sediment at the tip of the reaction vial, taken up, mounted on a slide with ProLong Gold antifade reagent (Molecular Probes) and covered with a coverslip and sealed with custom nail polish.

The non-bound biotinylated Antibodies were removed by $2 \times 5$ ' washing with PBT. For the coupling of Horseradish peroxidase (HRP) to the biotinylated secondary antibodies, the ABC-Kit Vectastain (Vector Laboratories) was used according to the manufacturers instruction. For HRP detection, the embryos were transferred into $500 \mu \mathrm{l}$ PBT including $10 \mu \mathrm{l}$ Diamino-Benzidine (10 mg/ml in $50 \mathrm{mM}$ Tris-HCl; Sigma) and $10 \mu \mathrm{l}$ hydrogenperoxide $(0,3 \%$ in PBT; Fluka). The embryos were then stepwise dehydrated $\left(2 \times 10^{\prime}\right.$ in Ethanol $70 \%$ and $2 \times 10^{\prime}$ in Ethanol 100\%), mounted on a slide with Canada balsam (Sigma), covered with a coverslip and sealed with custom nailpolish. 


\subsubsection{Microscope / Confocal imaging}

For taking Confocal images of embryos and cells, the Leica DMRXA2 Confocal Microscope (Leica Microsystems) was used. For image processing, the softwares ImageJ (National Institutes of Health) and Adobe Photoshop (Adobe Systems) were used.

\subsubsection{In vivo imaging of embryonic development}

For time lapse imaging of germ cell migration, $2 \mathrm{~h}$ collections of embryos were made. The embryos were arranged on an apple agar slice and fixed to a coverslip with embryo glue and covered with Halocarbon-Oil (Atochem) (protocol modified from germ line transfection protocol, section 2.2.1; Rubin and Spradling 1982). The coverslip was then applied on an Aluminum slide with a notch fitting the hanging drop with the embryos. Images of the developing embryos were taken from 1 to 12 hours.

For the measurement of cellularization speed, 30 min collections of embryos were treated as described above. For analysis, the Axiovert $200 \mathrm{M}$ (Leica) was used, which was operated with the software Openlab (Improvision). Data analysis was conducted with the software ImageJ (National Institutes of Health).

\subsubsection{Cuticle preparations}

$12 \mathrm{~h}$ embryo collections were aged in order to allow for complete hatching of viable embryos. The unhatched embryos were dechorionated, mounted in Hoyers Lactate medium $130 \mathrm{~g}$ gum Arabic, $\mathrm{H}_{2} \mathrm{O}$ to $50 \mathrm{ml}, 200 \mathrm{~g}$ chloral hydrate $20 \mathrm{~g}$ Glycerol, $150 \mathrm{ml}$ lactic acid) (modified from Stern and Sucena, 2000) and incubated at $60^{\circ} \mathrm{C}$ overnight. 


\subsection{Materials}

\subsubsection{Bacterial strains}

\begin{tabular}{c|l|l} 
E.coli-strain & reference & Remarks \\
\hline Dh5a & Invitrogen & for DNA amplification \\
\hline XL1-blue & Stratagene & for DNA amplification \\
\hline Top 10 & Invitrogen & for amplification of Gateway derived DNA \\
\hline BL-21 (DE3) & Stratagene & for heterologous protein expression
\end{tabular}

\subsubsection{Primers}

\begin{tabular}{|c|c|c|c|}
\hline Primer \# & Sequence & TM & $\begin{array}{l}\text { Rest. } \\
\text { Sites }\end{array}$ \\
\hline RGO043 & 5'-CGG ATG GGC TGA CTG GGA TGG-3' & 68,0 & \\
\hline RGO044 & 5'-CCT TTT CCT TCA CAG TAT GCC AAC A-3', & 72,1 & \\
\hline RGO045 & 5'-CGG GAT CCA CTG TGG TGG TGT CGA CAG CTT C-3', & $>75$ & BamHI \\
\hline RGO047 & 5'-CCC CAA GCT ICG ATG GAG GAC CAA AGC AAC G-3', & $>75$ & Hindlll \\
\hline RGO053 & 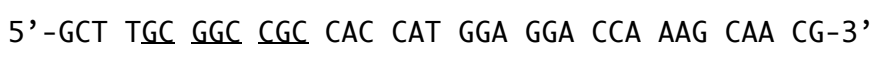 & $>75$ & Not I \\
\hline RGO054 & 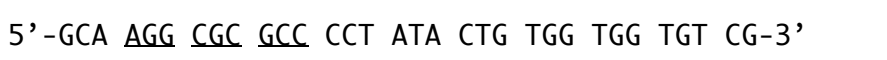 & $>75$ & Ascl \\
\hline RGO056 & 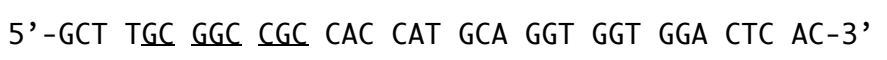 & $>75$ & Not 1 \\
\hline RGO059 & 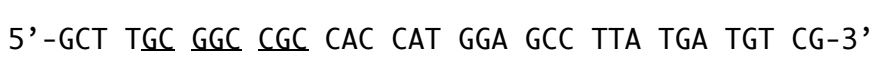 & $>75$ & Not I \\
\hline RGO060 & 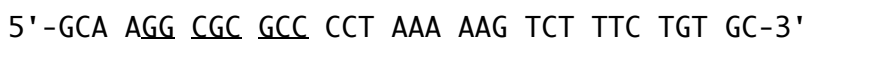 & 72,1 & Asc 1 \\
\hline RGO085 & 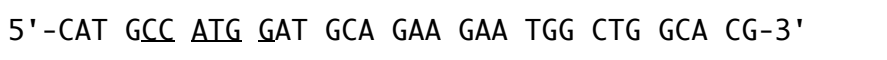 & $>75$ & $\mathrm{Ncol}$ \\
\hline RGO086 & $5^{\prime}-\mathrm{CCG} \underline{\mathrm{CTC}} \underline{\mathrm{GAG}}$ CTA GAT GCG CCA GCC CTG G-3' & $>75$ & Xhol \\
\hline RGO119 & 5'-GCT TGC GGC CGC CAC CAT GCC AGA AAG CCA CAG TT-3' & $>75$ & Not 1 \\
\hline RGO120 & $5^{\prime}-\mathrm{GCA}$ AGG $\underline{\mathrm{CGC}} \underline{\mathrm{GCC}}$ CTC AGA CCT CCA CGG CCC TCC-3' & $>75$ & Asc 1 \\
\hline RGO125 & 5'-CAT GAC CGC CCT GGA GTA-3' & 58,2 & \\
\hline
\end{tabular}




\begin{tabular}{|c|c|c|c|}
\hline Primer \# & Sequence & TM & $\begin{array}{l}\text { Rest. } \\
\text { sites }\end{array}$ \\
\hline RGO128 & $5^{\prime}-\mathrm{CTG}$ GAA GCC TAA TAT GCA GA-3' & 55,3 & \\
\hline RGO129 & 5'-ATG CAC CCC AAG TTC ACT GC-3' & 59,4 & \\
\hline RGO132 & $5^{\prime}-$ CTA TCG GGC ATG TTC ATC G-3' & 56,7 & \\
\hline RGO133 & $5^{\prime}-$ CTA TGG AGG ATG TGA GCC A-3' & 56,7 & \\
\hline RGO134 & $5^{\prime}-\mathrm{TGG}$ CTC ACA TCC TAC AGA G-3' & 56,7 & \\
\hline RGO135 & 5'-AGA TGA CCA GCG ACG AAT CC-3' & 59,4 & \\
\hline RGO137 & $5^{\prime}-\mathrm{TCA}$ TGT CGG CAT ACA CCC-3' & 56 & \\
\hline RGO140 & $5^{\prime}-\mathrm{CTA}$ GGA ACT GCG ACG AGG-3' & 58,2 & \\
\hline RGO141 & 5'-TCG GAA TGG ACG GAC AC-3', & 55,2 & \\
\hline RGO142 & $5^{\prime}-$ CTA GTG TCC GTC GAT TCC-3', & 56 & \\
\hline RGO143 & 5'-CTG GTG GGT ATG CAG ACA G-3, & 58,8 & \\
\hline RGO144 & $5^{\prime}-\mathrm{CTG}$ TCT ACA GAC CCA CCA G-3' & 58,8 & \\
\hline RGO147 & 5'-ATG CCA GAA AGC CAC AGT TAC-3' & 57,9 & \\
\hline RGO148 & $5^{\prime}-\mathrm{TCA}$ GAC CTC CAC GGC CC-3' & 60 & \\
\hline RGO154 & 5'-TCT GCA TAT ATG GCT TCC AGA-3, & 62,7 & \\
\hline RGO155 & 5'-GCA GTG AAC TAG GGG TGC TT-3' & 63,5 & \\
\hline RGO157 & 5'-GAG GGT GTA TGC CTA CTT GA-3' & 52,5 & \\
\hline RGO158 & 5'-CGC AGT TCC CTG GCC ATG-3', & 68,9 & \\
\hline RGO159 & 5'-CGA ATG CGC TAC TCC ACA-3', & 61,8 & \\
\hline RGO188 & 5'-TTC TCG CGA GTG CTG GTG A-3, & 58,8 & \\
\hline RGO189 & 5'-GTT TCC ACG CCC AGT AAT CA-3' & 57,3 & \\
\hline RGO200 & 5'-AGA AAT GTG TGC GTG CGA GTG-3' & 59,8 & \\
\hline RGO201 & 5'-CGT TGA TTA GGC TGG CTT CGG-3', & 61,8 & \\
\hline ,RGO227 & 5'-GAT CCT CAT GCC AAG TCC AG-3', & 63,8 & \\
\hline
\end{tabular}




\begin{tabular}{l|l|c|c} 
Primer \# & Sequence & TM & $\begin{array}{c}\text { Rest. } \\
\text { sites }\end{array}$ \\
\hline RGO228 & 5'-CCT AGG GCG TCT CTG GCT-3' & 63,8 & \\
\hline RGO234 & 5'-GCT TGC GGC CGC CAC CAT GTC GGC ATA CAC CCT CAC-3' & $>75$ & NotI \\
\hline RGO237 & 5'-GCA AGG CGC GCC CCT AGT GAG GGT CTA TGC CGA CAT-3, & $>75$ & AsCl
\end{tabular}

\subsubsection{Constructs}

\begin{tabular}{|c|c|c|}
\hline construct name & Purpose / Cloning strategy & reference \\
\hline pUAST-AenA & zygotic expression of AenA in flies & Molitor (2002) \\
\hline pUASP-AenA & maternal expression of AenA in flies & $\begin{array}{l}\text { Jochen Deckert } \\
\text { (this work) }\end{array}$ \\
\hline pUASP-AenA-fl-eGFP & $\begin{array}{l}\text { maternal expression of AenA-eGFP; } \\
\text { PCR with RGO045/047 on LD30155, } \\
\text { subcloning in pEGFP via BamHI- } \\
\text { Hindll, cloning in PUASP via } \\
\text { BamHI/Bgll-Xbal }\end{array}$ & $\begin{array}{l}\text { Roland Graf (this } \\
\text { work) }\end{array}$ \\
\hline $\begin{array}{l}\text { pCasper4-Aeneas- } \\
\text { gen-resc }\end{array}$ & $\begin{array}{l}\text { genomic rescue of aen mutants; } \\
\text { PCR with RGO043/044 on wt } \\
\text { genomic DNA, subcloning in pTopo- } \\
\mathrm{XL} \text {, cloning into pCasper4 via EcoRI }\end{array}$ & $\begin{array}{l}\text { Gerd } \\
\text { Vorbrüggen (this } \\
\text { work) }\end{array}$ \\
\hline pCITE-shg-ICD & $\begin{array}{l}\text { in vitro transcription/ translation of } \\
\text { shg-ICD; PCR with RGO085/086 on } \\
\text { PUAST-DEcadherin, cloning into } \\
\text { PCITE via Ncol-Xhol }\end{array}$ & $\begin{array}{l}\text { Roland Graf (this } \\
\text { work) }\end{array}$ \\
\hline pENTR-AenA-N & $\begin{array}{l}\text { gateway recombination of the } \\
\text { aenA cassette; PCR with } \\
\text { RG0053/054 on LD30155 and } \\
\text { cloning into pENTR-D via Notl-AsCl }\end{array}$ & $\begin{array}{l}\text { Roland Graf (this } \\
\text { work) }\end{array}$ \\
\hline
\end{tabular}




\begin{tabular}{|c|c|c|}
\hline construct name & Purpose / Cloning strategy & reference \\
\hline pENTR-AenB-N & $\begin{array}{l}\text { gateway recombination of the } \\
\text { aenB cassette; PCR with } \\
\text { RG0056/054 on RH35990 and } \\
\text { cloning into PENTR-D via Notl-Ascl }\end{array}$ & $\begin{array}{l}\text { Roland Graf (this } \\
\text { work) }\end{array}$ \\
\hline pENTR-Arp 1-N & $\begin{array}{l}\text { gateway recombination of the arp } 1 \\
\text { cassette; PCR with RGO059/060 on } \\
\text { RH04757 and cloning into PENTR-D } \\
\text { via Notl-AsCl }\end{array}$ & $\begin{array}{l}\text { Roland Graf (this } \\
\text { work) }\end{array}$ \\
\hline pENTR-SlamNt-N & $\begin{array}{l}\text { gateway recombination of the } \\
\text { slam-N-fragment cassette; PCR with } \\
\text { RGO119/237 on LD22808 and } \\
\text { cloning into PENTR-D via Notl-AsCl }\end{array}$ & $\begin{array}{l}\text { Roland Graf (this } \\
\text { work) }\end{array}$ \\
\hline pENTR-SlamCt-N & $\begin{array}{l}\text { gateway recombination of the } \\
\text { slam-C-fragment cassette; PCR with } \\
\text { RGO120/234 on LD22808 and } \\
\text { cloning into pENTR-D via Notl-AsCl }\end{array}$ & $\begin{array}{l}\text { Roland Graf (this } \\
\text { work) }\end{array}$ \\
\hline pETG-TrxA-AenA & $\begin{array}{l}\text { expression of TrxA-AenA in BL21 } \\
\text { (DE3); gateway recombination of } \\
\text { pENTR-AenA-N with pETG-20A }\end{array}$ & $\begin{array}{l}\text { Roland Graf (this } \\
\text { work) }\end{array}$ \\
\hline pETG-MBP-AenA & $\begin{array}{l}\text { expression of MBP-AenA in BL21 } \\
\text { (DE3); gateway recombination of } \\
\text { PENTR-AenA-N with pETG-40A }\end{array}$ & $\begin{array}{l}\text { Roland Graf (this } \\
\text { work) }\end{array}$ \\
\hline pETG-NusA-His6-AenA & $\begin{array}{l}\text { expression of NusA-His6-AenA in } \\
\text { BL21 (DE3); gateway recombination } \\
\text { of pENTR-AenA-N with pETG-60A }\end{array}$ & $\begin{array}{l}\text { Roland Graf (this } \\
\text { work) }\end{array}$ \\
\hline pETG-His6-GST-AenA & $\begin{array}{l}\text { expression of His6-GST-AenA in BL21 } \\
\text { (DE3); gateway recombination of } \\
\text { pENTR-AenA-N with pETG-30A }\end{array}$ & $\begin{array}{l}\text { Roland Graf (this } \\
\text { work) }\end{array}$ \\
\hline pETG-30A-df-ccdB & $\begin{array}{l}\text { expression of His6-GST in BL21 (DE3); } \\
\text { from pETG-30A by excision of the } \\
\text { ccdb cassette with Kpnl-Xhol, } \\
\text { blunting and religation }\end{array}$ & $\begin{array}{l}\text { Roland Graf (this } \\
\text { work) }\end{array}$ \\
\hline
\end{tabular}




\begin{tabular}{|c|c|c|}
\hline construct name & Purpose / Cloning strategy & reference \\
\hline pUbiP-10xMyc-Arpl & $\begin{array}{l}\text { expression of } 10 x M y c-A r p l \text { in cell } \\
\text { culture; gateway recombination of } \\
\text { pENTR-Arp1-N with pUbiP-10xMyc- } \\
\text { rfa }\end{array}$ & $\begin{array}{l}\text { Roland Graf (this } \\
\text { work) }\end{array}$ \\
\hline $\begin{array}{l}\text { pUbiP-10xMyc - } \\
\text { SlamNterm }\end{array}$ & $\begin{array}{l}\text { For expression of } 10 x M y c-S l a m-N- \\
\text { fragment in cell culture; gateway } \\
\text { recombination of pENTR-SlamNt-N } \\
\text { with pUbiP-10xMyc-rfa }\end{array}$ & $\begin{array}{l}\text { Roland Graf (this } \\
\text { work) }\end{array}$ \\
\hline $\begin{array}{l}\text { pUbiP-10xMyc- } \\
\text { SlamCterm }\end{array}$ & $\begin{array}{l}\text { expression of 10xMyc-Slam-C- } \\
\text { fragment in cell culture; gateway } \\
\text { recombination of pENTR-SlamCt-N } \\
\text { with pUbiP-10xMyc-rfa }\end{array}$ & $\begin{array}{l}\text { Roland Graf (this } \\
\text { work) }\end{array}$ \\
\hline pUbiP-3xFLAG-AenA & $\begin{array}{l}\text { expression of } 3 x F L A G-A e n A \text { in cell } \\
\text { culture; gateway recombination of } \\
\text { pENTR-AenA-N with pUbiP-3xFLAG - } \\
\text { rfa }\end{array}$ & $\begin{array}{l}\text { Roland Graf (this } \\
\text { work) }\end{array}$ \\
\hline pUbiP-3xFLAG-AenB & $\begin{array}{l}\text { expression of } 3 x F L A G-A e n B \text { in cell } \\
\text { culture; gateway recombination of } \\
\text { pENTR-AenB-N with pUbiP-3xFLAG - } \\
\text { rfa }\end{array}$ & $\begin{array}{l}\text { Roland Graf (this } \\
\text { work) }\end{array}$ \\
\hline pCMVTNT-SlamFragl & $\begin{array}{l}\text { in vitro transcription/ translation of } \\
\text { bp 1-600 of the slam ORF; PCR with } \\
\text { RGO147/128 on LD22808, } \\
\text { subcloning in pCRII-Topo and } \\
\text { cloning into PCMVTNT via ECORI }\end{array}$ & $\begin{array}{l}\text { Roland Graf (this } \\
\text { work) }\end{array}$ \\
\hline pCMVTNT-SlamFrag2 & $\begin{array}{l}\text { For in vitro transcription/ translation } \\
\text { of bp 303-920 of the slam ORF; PCR } \\
\text { with RGO125/155 on LD22808, } \\
\text { further strategy see above }\end{array}$ & $\begin{array}{l}\text { Roland Graf (this } \\
\text { work) }\end{array}$ \\
\hline
\end{tabular}




\begin{tabular}{|c|c|c|}
\hline construct name & Purpose / Cloning strategy & reference \\
\hline pCMVTNT-SlamFrag3 & $\begin{array}{l}\text { in vitro transcription/ translation of } \\
\text { bp 610-1230 of the slam ORF; PCR } \\
\text { with RGO154/132 on LD22808, } \\
\text { further strategy see above }\end{array}$ & $\begin{array}{l}\text { Roland Graf (this } \\
\text { work) }\end{array}$ \\
\hline pCMVTNT-SlamFrag4 & $\begin{array}{l}\text { in vitro transcription/ translation of } \\
\text { bp 901-1532 of the slam ORF; PCR } \\
\text { with RG0129/134 on LD22808, } \\
\text { further strategy see above }\end{array}$ & $\begin{array}{l}\text { Roland Graf (this } \\
\text { work) }\end{array}$ \\
\hline pCMVTNT-SlamFrag5 & $\begin{array}{l}\text { in vitro transcription/ translation of } \\
\text { bp } 1140-1838 \text { of the slam ORF; PCR } \\
\text { with RGO227/228 on LD22808, } \\
\text { further strategy see above }\end{array}$ & $\begin{array}{l}\text { Roland Graf (this } \\
\text { work) }\end{array}$ \\
\hline pCMVTNT-SlamFrag6 & $\begin{array}{l}\text { in vitro transcription/ translation of } \\
\text { bp 1514-2128 of the slam ORF; PCR } \\
\text { with RGO134/157 on LD22808, } \\
\text { further strategy see above }\end{array}$ & $\begin{array}{l}\text { Roland Graf (this } \\
\text { work) }\end{array}$ \\
\hline pCMVTNT-SlamFrag7 & $\begin{array}{l}\text { in vitro transcription/ translation of } \\
\text { bp } 1799-2422 \text { of the slam ORF; PCR } \\
\text { with RG0135/158 on LD22808, } \\
\text { further strategy see above }\end{array}$ & $\begin{array}{l}\text { Roland Graf (this } \\
\text { work) }\end{array}$ \\
\hline pCMVTNT-SlamFrag8 & $\begin{array}{l}\text { in vitro transcription/ translation of } \\
\text { bp } 2111-2721 \text { of the slam ORF; PCR } \\
\text { with RGO137/142 on LD22808, } \\
\text { further strategy see above }\end{array}$ & $\begin{array}{l}\text { Roland Graf (this } \\
\text { work) }\end{array}$ \\
\hline PCMVTNT-SlamFrag9 & $\begin{array}{l}\text { in vitro transcription/ translation of } \\
\text { bp 2410-3019 of the slam ORF; PCR } \\
\text { with RG0158/144 on LD22808, } \\
\text { further strategy see above }\end{array}$ & $\begin{array}{l}\text { Roland Graf (this } \\
\text { work) }\end{array}$ \\
\hline pCMVTNT-SlamFrag 10 & $\begin{array}{l}\text { in vitro transcription/ translation of } \\
\text { bp } 2702-3326 \text { of the slam ORF; PCR } \\
\text { with RGO141/159 on LD22808, } \\
\text { further strategy see above }\end{array}$ & $\begin{array}{l}\text { Roland Graf (this } \\
\text { work) }\end{array}$ \\
\hline
\end{tabular}




\begin{tabular}{|c|c|c|}
\hline construct name & Purpose / Cloning strategy & reference \\
\hline pCMVTNT-SlamFrag 11 & $\begin{array}{l}\text { in vitro transcription/ translation of } \\
\text { bp } 3001-3591 \text { of the slam ORF; PCR } \\
\text { with RGO143/148 on LD22808, } \\
\text { further strategy see above }\end{array}$ & $\begin{array}{l}\text { Roland Graf (this } \\
\text { work) }\end{array}$ \\
\hline \multicolumn{3}{|c|}{ Original vectors used for cloning or recombination } \\
\hline PUAST & $\begin{array}{l}\text { generation of transgenes and } \\
\text { zygotic expression }\end{array}$ & $\begin{array}{l}\text { Brand and } \\
\text { Perrimon, } 1993\end{array}$ \\
\hline PUASP & $\begin{array}{l}\text { generation of transgenes and } \\
\text { maternal expression }\end{array}$ & Rorth, 1998 \\
\hline pCaspeR4 & generation of transgenes & $\begin{array}{l}\text { Klemenz et al., } \\
1987\end{array}$ \\
\hline PENTR-D/TOPO & generation of entry-vectors & Invitrogen \\
\hline pEGFP-NI & generation of eGFP-fusion proteins & Clontech \\
\hline PCITE-1 $(+)$ & efficient transcription & Novagen \\
\hline pETG-20A (TrXA-rfa) & $\begin{array}{l}\text { bacterial expression of TrxA-fusion } \\
\text { proteins }\end{array}$ & $\begin{array}{l}\text { A. Geerlof } \\
\text { (EMBL) }\end{array}$ \\
\hline pETG-40A (MBP-rfa) & $\begin{array}{l}\text { bacterial expression of MBP-fusion } \\
\text { proteins }\end{array}$ & $\begin{array}{l}\text { A. Geerlof } \\
\text { (EMBL) }\end{array}$ \\
\hline $\begin{array}{l}\text { pETG-60A (NusA-His6- } \\
\text { rfa) }\end{array}$ & $\begin{array}{l}\text { bacterial expression of His6-NusA- } \\
\text { fusion proteins }\end{array}$ & $\begin{array}{l}\text { A. Geerlof } \\
\text { (EMBL) }\end{array}$ \\
\hline $\begin{array}{l}\text { pETG.30A (His6-GST- } \\
\text { rfa) }\end{array}$ & $\begin{array}{l}\text { bacterial expression of His6-Gst- } \\
\text { fusion proteins }\end{array}$ & $\begin{array}{l}\text { A. Geerlof } \\
\text { (EMBL) }\end{array}$ \\
\hline pUbiP-10xMyc-rfa & $\begin{array}{l}\text { expression of } 10 x M y c \text {-fusion proteins } \\
\text { in culture cells }\end{array}$ & $\begin{array}{l}\text { generous gift } \\
\text { from A. Herzig }\end{array}$ \\
\hline pUbiP-3xFLAG-rfa & $\begin{array}{l}\text { expression of } 3 x F L A G \text {-fusion proteins } \\
\text { in culture cells }\end{array}$ & $\begin{array}{l}\text { generous gift } \\
\text { from A. Herzig }\end{array}$ \\
\hline PCMVTNT & $\begin{array}{l}\text { Efficient in vitro } \\
\text { transcription/translation }\end{array}$ & Promega \\
\hline
\end{tabular}




\subsection{4 cDNA vectors}

\begin{tabular}{c|c|c|c} 
Gene & CDNA-ID & vector & reference \\
\hline aenA & LD30155 & pOT2 & DGRC \\
\hline aenB & RH35990 & pFLC-1 & DGRC \\
\hline arpl & RH04757 & pFLC-1 & DGRC \\
\hline slam & LD22808 & pCS+ & Lecuit et al., 2002 \\
\hline arp 1 1 & RH13978 & pFLC-1 & DGRC \\
\hline capz & RE71950 & pFLC-1 & DGRC
\end{tabular}

\subsubsection{Fly stocks}

Following fly stocks were used in this study:

w 1118 (Bloomington \#5905), w*; ${ }^{*}$ aen² (Molitor, 2002), Df(3R)XM3, rul thl $\mathrm{st}^{1}$ $k_{n i r-1} C U^{1} p^{p} e^{1 / T M 3}, S b^{1}$ (Bloomington \# 1655), $w^{*} ; P\left\{W^{+m C}=V A S A G F P\right\} ; a e n^{2}$ (Gerd Vorbrüggen, this work), $W^{*} ; ; P\left\{W^{+m C}=U A S T-a e n A\right\} \# 1.1 \quad$ (Gerd Vorbrüggen, this work), $W^{*} ; ; P\left\{W^{+m C}=U A S T-a e n A\right\} \# 1.1$, aen ${ }^{2}$ (Roland Graf, this work), W*;; P\{w-mCUASp-aenA-fl-eGFP\}\# 1 (Roland Graf, this work), w*; P\{W-mCUASp-aenA-fl-eGFP\}\#17 (Roland Graf, this work), W*;UAS-SlamHA/TM6C, AbdZ (kindly provided by Thomas Lecuit), w*; wdo20.84 m/Cyo, ftzlacZ (kindly provided by Ruth Lehmann), w'118;Df(2L)ED369, Df(2L)ED369, $P\left\{W^{+m W . S c e r} \vee F R T . h s 3=3\right.$ '.RS5+3.3' $\} E D 369 / C y O, h b-l a c z$ (Bloomington \#8903), w*;wdo $20.84 \mathrm{~m} / \mathrm{Cyo}$,ftz-lacz;aen² (Roland Graf, this work), w*:Df(2L)ED369,

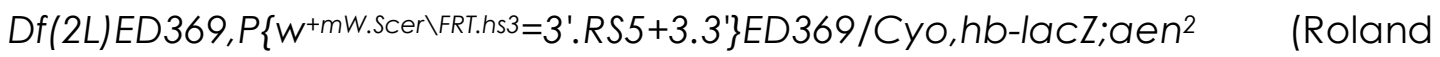
Graf, this work) 
2.5.6 Antibodies used for Western blot detection and coimmunoprecipitation

\begin{tabular}{c|c|c|c} 
antibody & origin & $\begin{array}{c}\text { working } \\
\text { concentration }\end{array}$ & reference \\
\hline $\begin{array}{c}\text { anti-( } \beta \text { )Tubulin } \\
\text { (E7) }\end{array}$ & $\begin{array}{c}\text { mouse- } \\
\text { monoclonal }\end{array}$ & $1: 1000$ & Roland Graf (this work) \\
\hline anti-Myc-E10 & $\begin{array}{c}\text { mouse- } \\
\text { monoclonal }\end{array}$ & $\begin{array}{c}1: 10 \\
\text { undiluted (resin } \\
\text { loading) }\end{array}$ & lowa-Hybridoma bank \\
\hline anti-Flag (M2) & $\begin{array}{c}\text { mouse- } \\
\text { monoclonal }\end{array}$ & $1: 1000$ & Sigma \\
\hline HRP anti-rabbit & goat & $1: 4000$ & Pierce \\
\hline HRP anti-mouse & goat & $1: 4000$ & Pierce
\end{tabular}

The anti-( $\beta$ )Tubulin (E7) antibody developed by Michael Klymkowsky and the anti-C-Myc (9E10) antibody developed by J. Michael Bishop were obtained from the Developmental Studies Hybridoma Bank developed under the auspices of the NICHD and maintained by The University of lowa, Department of Biological Sciences, lowa City, IA 52242.

2.5.7 Antibodies used in immunohistological stainings

\begin{tabular}{c|c|c|c} 
antibody & origin & $\begin{array}{c}\text { Working } \\
\text { concentration }\end{array}$ & reference \\
\hline anti-Aen & rabbit & $1: 200$ & Roland Graf (this work) \\
\hline anti-Tubulin-E7 & $\begin{array}{c}\text { mouse- } \\
\text { monoclonal }\end{array}$ & $1: 50$ & lowa-Hybridoma bank \\
\hline anti-Lava Lamp & rabbit & $1: 1000$ & Sisson et al., 2000
\end{tabular}




\begin{tabular}{|c|c|c|c|}
\hline antibody & origin & $\begin{array}{c}\text { working } \\
\text { concentration }\end{array}$ & reference \\
\hline anti-Rabl1 & rat & $1: 100$ & Emery et al., 2005 \\
\hline $\begin{array}{l}\text { anti-Dynein } \\
\text { Heavy Chain }\end{array}$ & mouse & $1: 100$ & McGrail and Hays, 1997 \\
\hline anti-HA & mouse & 1:2000 & $\begin{array}{l}\text { generous gift from R. } \\
\text { Jahn }\end{array}$ \\
\hline anti-Discs lost & rabbit & $1: 500$ & Bhat et al., 1999 \\
\hline anti-Nrt (BP106) & $\begin{array}{l}\text { mouse- } \\
\text { monoclonal }\end{array}$ & $1: 10$ & lowa-Hybridoma bank \\
\hline $\begin{array}{l}\text { Alexa Fluor } 488 \\
\text { anti-rabbit }\end{array}$ & goat & $1: 400$ & Invitrogen \\
\hline $\begin{array}{l}\text { Alexa Flour } 568 \\
\text { anti-rabbit }\end{array}$ & goat & $1: 400$ & Invitrogen \\
\hline $\begin{array}{l}\text { Alexa Flour } 488 \\
\text { anti-mouse }\end{array}$ & goat & $1: 400$ & Invitrogen \\
\hline $\begin{array}{l}\text { Alexa Flour } 568 \\
\text { anti-mouse }\end{array}$ & goat & $1: 400$ & Invitrogen \\
\hline $\begin{array}{c}\text { Cyanine Cy3 } \\
\text { anti-rat }\end{array}$ & goat & $1: 400$ & $\begin{array}{c}\text { Jackson } \\
\text { Immunoresearch }\end{array}$ \\
\hline $\begin{array}{l}\text { Biotinylated anti- } \\
\text { rabbit }\end{array}$ & goat & $1: 400$ & Vector Laboratories \\
\hline
\end{tabular}

The anti-Nrt (BP106) antibody developed by Corey Goodman was obtained from the Developmental Studies Hybridoma Bank developed under the auspices of the NICHD and maintained by The University of lowa, Department of Biological Sciences, lowa City, IA 52242.

The antibody anti-Discs lost, which was formerly described to detect the protein Dlt (Bhat et al., 1999) has been shown to specifically detect D-PATJ instead (Pielage et al., 2003). In this study the anti-Discs lost antibody was used for the detection of D-PATJ. 


\section{Results}

\subsection{Conservation of the Aeneas proteins}

The conservation of a protein provides important indications towards its function and structure and its evolutionary age might be indicative of its requirement.

To identify functional domains in the Aeneas (Aen) protein sequence, an analysis using the SMART protein analysis tool (http://smart.emblheidelberg.de) was conducted, using the AenA protein sequence as input. The result showed that AenA features no domains, which have been functionally characterized.

Therefore, the conservation of Aen within the family of Drosophilids was investigated to identify regions of high conservation. This analysis encompassed known or predicted Aen protein sequences from 11 Drosophila species covering an evolutionary distance of about 40 Mio Years (Ashburner et al., 1989). The sequences were identified by BLAT search (http://genome.ucsc.edu/index.html) and compared utilizing the software-tool Evoprinter (http://evoprinter.ninds.nih.gov). The analysis showed that two regions exist in the Aen sequence that are highly conserved within the Drosophilid family (figure 6b). The conserved $\mathrm{N}$-terminal region covers 294 a a from residue 25 to 318 and a carboxyterminal (C-terminal) region of 41 a covering residue 416 to 456 of the Drosophila melanogaster AenA protein.

A detailed analysis of the regions conserved between Drosophilids revealed that Aen is a member of a highly conserved protein family. 


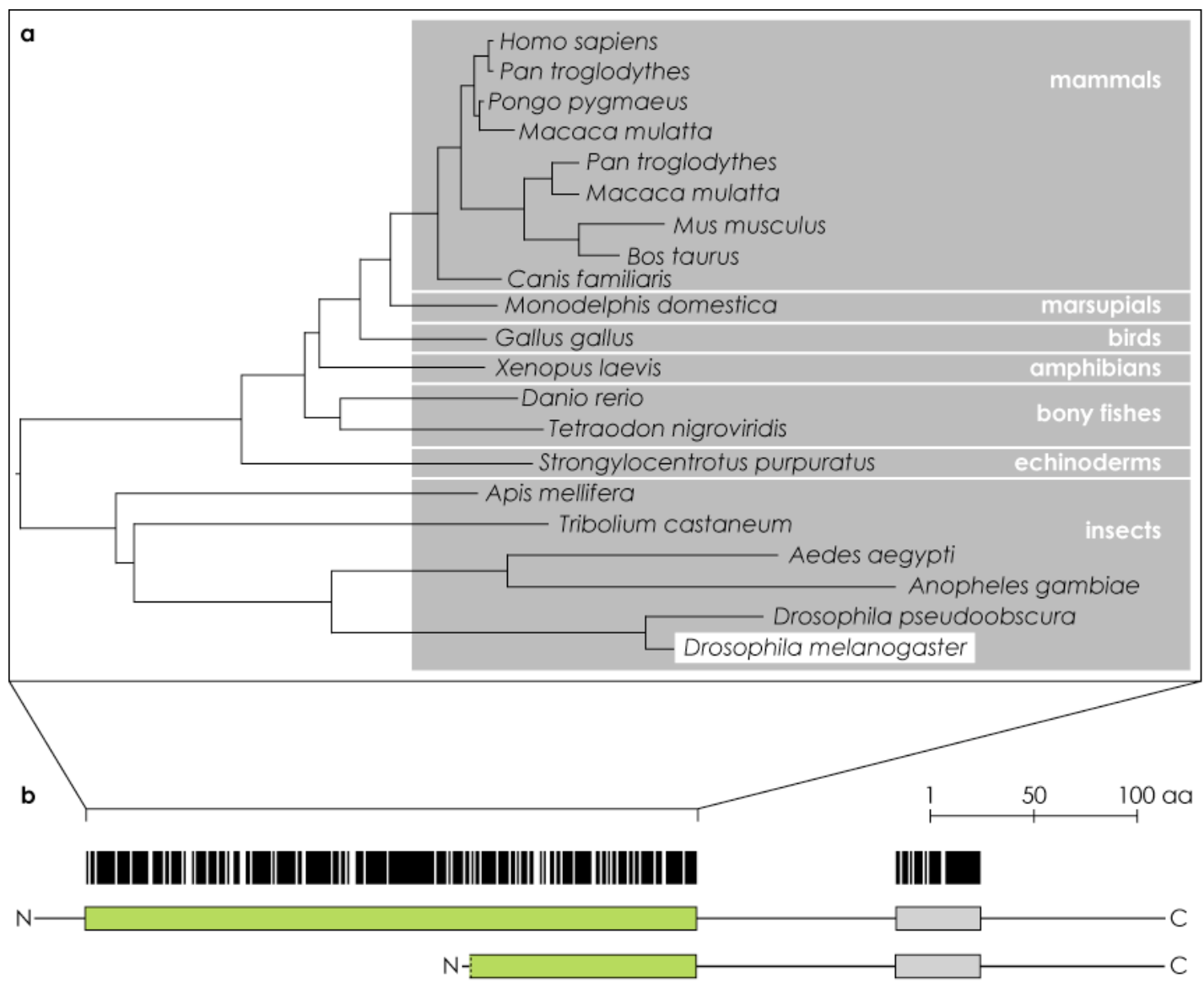

Figure 6: Conservation of the Aen proteins. a) Conservation of the $\mathrm{N}$-terminal domain of the Aen proteins in the animal kingdom. b) Conserved residues of Aen in Drosophilids. Vertical lines mark residues conserved between D. melanogaster, D. simulans, D. yakuba, D. erecta, D. perisimilis, D. sechellia, D. mojavensis, D. pseudoobscura, D. virilis, D. ananassae and D. grimshawi as determined with the evoprinter tool. Green: highly conserved $\mathrm{N}$-terminal domain of Aen. Light grey: C-terminal region, conserved whithin Drosophilids.

Sequences of potential Aen homologs were retrieved by analyzing the protein sequence of the conserved $\mathrm{N}$ - and C-terminal Aen domains with the blastp algorithm using standard parameters (BLOSUM62 scoring matrix) (www.ncbi.nlm.nih.gov). It was found that the Cterminal region is only conserved within Drosophilids (data not shown), whereas the long $\mathrm{N}$-terminal conserved region exhibits a high degree of conservation in a wide range of species. A phylogenetic tree was generated from the retrieved sequences with the BLAST Tree Widget, using the Fast Minimum Evolution method with a difference cutoff of 0.7 (figure 6a; for BLAST results see section 2.1.8). It shows that the Aen protein is conserved throughout the animal kingdom. A detailed analysis of the Aen homologs suggests that in primates at least two protein variants exist, which arise from alternative usage of an internal 
exon. Other potential splice variants, representing aminoterminal ( $\mathrm{N}$ terminal) truncations were not included in the phylogenetic tree because the incompleteness of the transcriptome and proteome data in most organisms does not allow for their secure prediction.

The high conservation of Aen indicates that its function is required for animal life.

\subsection{Characterization of the phenotype observed in aen mutant embryos}

The aen phenotype was analyzed in two aen loss of function (LOF) allelic combinations, in aen2 and in aen2/Df(3R)XM3 transheterozygous embryos (deficiency Df(3R)XM3 covering cytogenetic bands 082A0306;082B including the aen locus). The aen ${ }^{2}$ allele was generated by imprecise remobilization a P-element insertion in the first intron of both aen transcripts (figure 5b) in the germline of the aen' mutant (Molitor, 2002). In aen ${ }^{2}$ mutants, the remobilization resulted in a deletion of 1476 bp that removes the complete second and part of the third exon of the aen gene (figure $5 b$ ), which are included in both aen transcripts, aen $A$ and $a e n B$. Despite the fact that the promoter regions and the transcriptional start sites of both aen transcripts were not affected by the deletion, the removal of major parts of the open reading frames suggested that aen is a null allele or a strong hypomorphic allele. Furthermore, flies carrying the aen² allele are viable and fertile, which allowed the investigation of the aen LOF phenotype in the offspring of homozygous aen ${ }^{2}$ mutant parents. Therefore, the aen mutant phenotypes presented throughout this work represent a maternal and zygotic null mutant or strong hypomorphic situation. 
3.2.1 aen mutant embryos show a strong increase in untimely PGC migration

For the analysis 2-4 hours old aen mutant embryos and wildtype embryos were collected, fixed and subjected to immunohistochemical stainings using an anti-Vasa antibody, which specifically marks the primordial germ cells (PGCs) (Jaglarz and Howard, 1995). The number of PGCs that were present in ectopic positions was counted. During blastoderm stage, ectopic PGCs are found either intercalated between the forming somatic blastoderm cells or in one plane with the progressing furrow canal (FC) (figure $7 \mathrm{~b}$ and 7b'). It was found that untimely transmigration of PGCs through the PMG can be detected at earliest at stage 5-3 during blastoderm cellularization, after the FC has passed the nuclei of the somatic blastoderm. Ectopic PGCs occur both in wildtype and in aen mutant embryos. Therefore, the numbers of wildtype (wt) embryos exhibiting different phenotypic intensities were counted in order to determine the phenotypic background present in the assayed system. Phenotypic intensities were characterized by the number of mismigrating PGCs. It was found that mismigration of 3 or more PGCs occurs in less than $10 \%$ of wt embryos (figure 7c). Therefore, the phenotypic intensity of 0 to 2 ectopic PGCs per embryo was defined as a cutoff criterion for wt versus mutant phenotypes.

Subsequently, the ratio of embryos exhibiting wt and mutant phenotypes were analyzed in homozygous aen ${ }^{2}$ mutants, in transheterozygous aen mutants bearing the aen ${ }^{2}$ allele in trans to the deficiency $D f(3 R) X M 3$ and in wt. The results of the subsequent analysis show that in wt, $92 \%$ of the embryos show 0-2 transmigrating PGCs while only $8 \%$ have a higher number of mismigrating PGCs (figure 7d, left). In contrast, $44 \%$ of aen² mutant embryos exhibit a strong PGC transmigration phenotype while the effect is weak in $56 \%$ (figure $7 d$, 


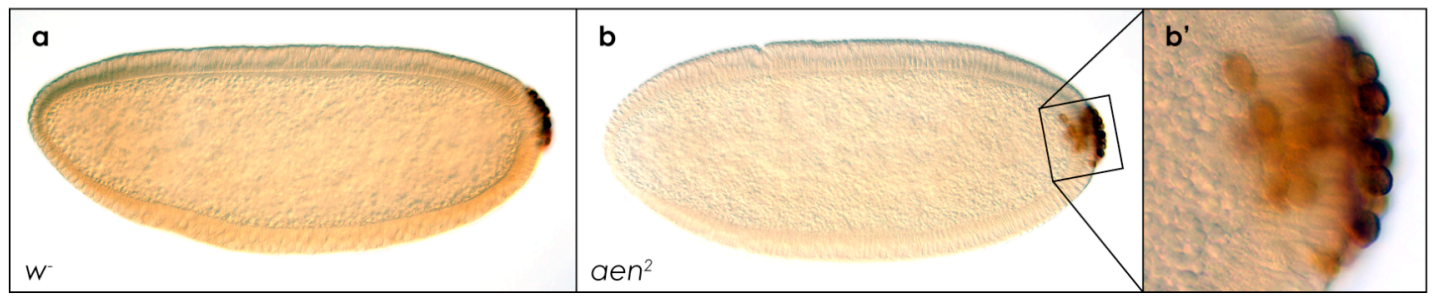

C

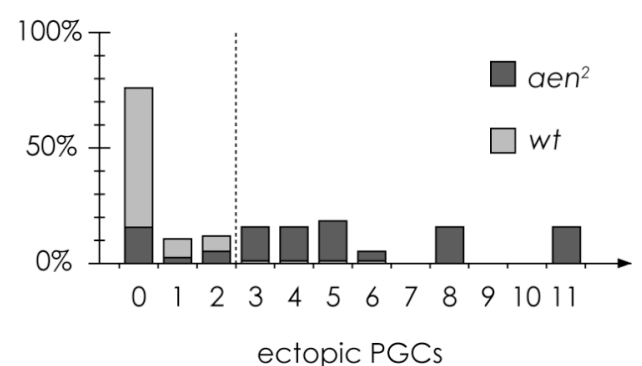

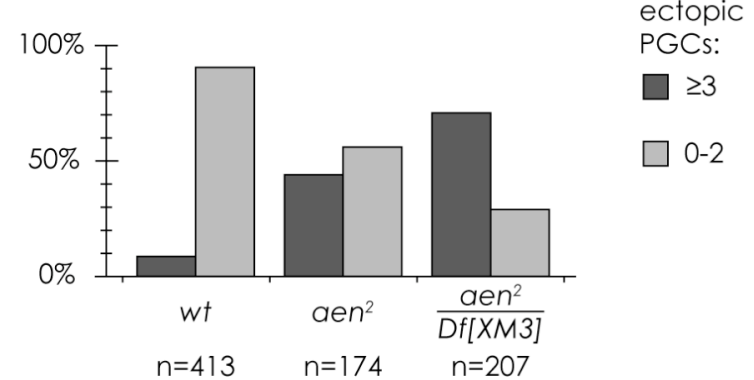

e

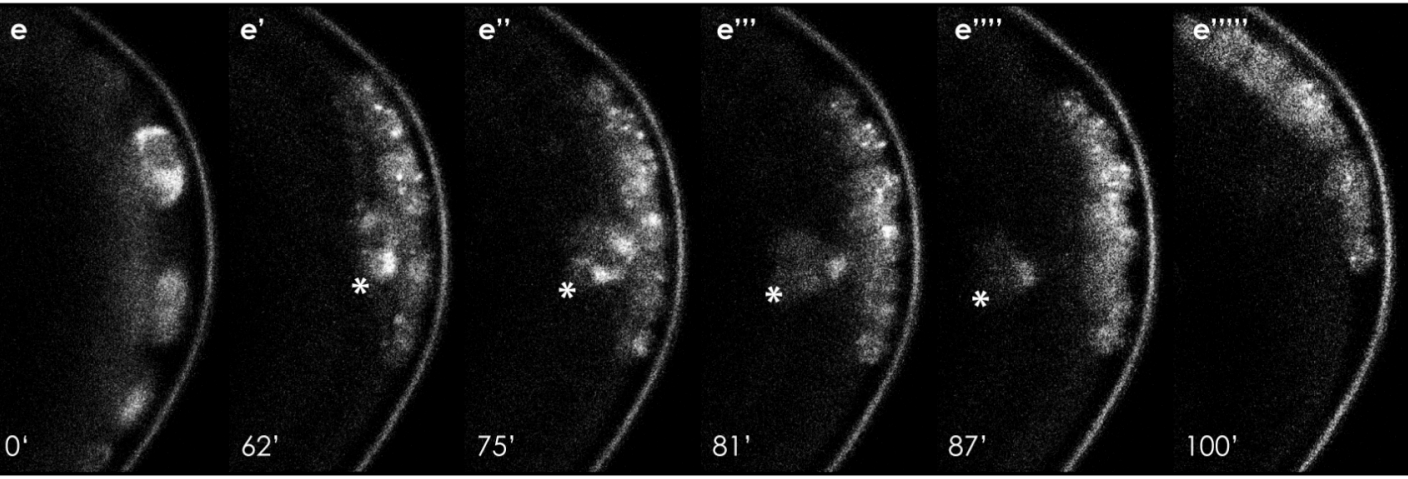

Figure 7: Phenotype of aen mutants. a) wt embryo; stage 5-3; PGCs (dark brown) stained with anti-Vas. b) aen ${ }^{2}$ embryo stained with anti-Vas. b') enlargement of the posterior pole of an aen ${ }^{2}$ embryo. c) Definition of phenotypic background. Measurement of percentage of stage 5-3 wt and aen ${ }^{2}$ mutant embryos exhibiting increased numbers of transmigrating PGCs. Dashed line indicates threshold between weak $10-2$ ectopic PGCs) and strong ( $\geq 3$ ectopic PGCs) phenotypes. d) PGC transmigration at stage 5-3 in wt (left), homozygous aen ${ }^{2}$ mutant (middle) and aen ${ }^{2}$ mutant in trans over the deficiency Df[XM3] (right); dark: strong phenotype, light no/weak phenotype. $n$ denotes the number of analyzed embryos. e) PGC transmigration in aen ${ }^{2}$ mutants in vivo. PGCs marked with Vas-GFP. Confocal time-lapse frames. Numbers indicate lapsed time in minutes. e) Budding of PGCs. e'-e"',') Blastoderm cellularization. Cluster of transmigrating PGCs (asterisks). e"',"') Gastrulation.

middle). A comparison of aen² homozygous mutant embryos with the offspring of aen²/Df[XM3] transheterozygous mutant females, crossed to aen ${ }^{2}$ homozygous males, shows that the distribution is shifted towards strong phenotypes in the transheterozygous situation. While 70 $\%$ of the aen mutant embryos exhibit a strong PGC transmigration phenotype, weak in phenotypic intensities are found in $30 \%$ (figure $7 d$, right).

This result indicates that the aen² allele might exhibit residual gene activity. However the deletion of $1476 \mathrm{bp}$ in the aen ${ }^{2}$ allele removes 
most of the ORF and reduces the transcript and protein amounts to undetectable levels (Molitor, 2002; see below) suggesting that aen² represents a null allele.

\subsubsection{In vivo imaging of PGC migration in aen mutant embryos}

The morphology of the PGCs observed in the anti-Vasa stainings of aen ${ }^{2}$ mutant embryos is similar to the one found in PGCs actively transmigrating the PMG at stage 10. The formerly spherical PGCs form filopodia that intercalate in between the underlying epithelial cells. The bodies of cells crossing the forming epithelium exhibit an elongated, spindle-shaped form and readopt a spherical shape once they reach the body cavity (figure 7b'). These observations give a first indication that the cells might actively migrate into the epithelium, however these stainings reflect only a static image of a dynamic process. To clarify in which way the ectopic PGCs become separated from the PGC cluster. Time-lapse movies of living aen ${ }^{2}$ embryos carrying a Vasa-eGFP construct under the control of the endogenous vasa-promoter were taken (Breitwieser et al., 1996). The Vasa-eGFP fusion protein, which labels the PGCs, was used to track PGC development from their budding out at stage 3 to germ band extension at stage 6 (see stills in figure $7 e$ ).

The recorded image series shows that pole cap formation and morphology are normal in aen ${ }^{2}$ mutant embryos until the beginning of stage 5-2, approximately $60 \mathrm{~min}$ after budding (figure 7e'). At this point, several PGCs that are positioned basally in the PGC cluster undergo a change in cell shape, elongating in apicobasal direction in respect to the underlying epithelium (figure 7e" '). Thereby they start to translocate in basal direction. This movement continues for the following minutes (figure $7 \mathrm{e}^{\prime \prime}$ '), leading to an ectopic positioning of the PGCs inside the somatic blastoderm (figure 7e"' '). By the start of gastrulation, the ectopic PGCs are no longer detected (figure 7e'"','). 


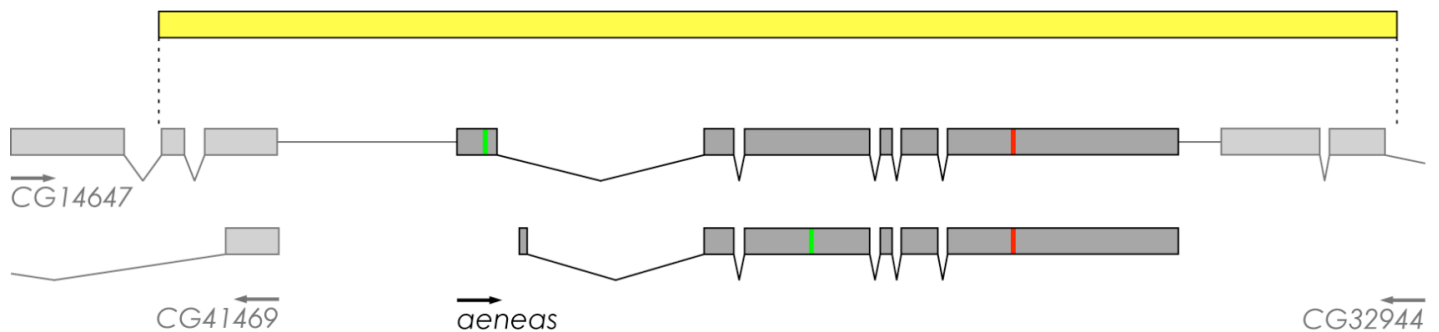

b

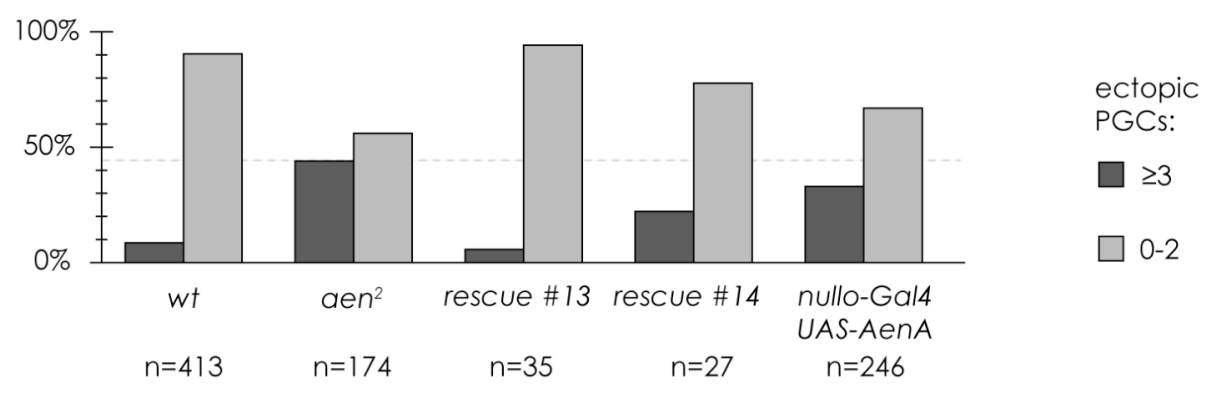

Figure 8: Rescue of PGC transmigration phenotypes in aen ${ }^{2}$ mutants. a) Coverage of the aen genomic rescue fragment, extending over the aen genomic sequence, to the 2. exon of the gene CG14647 at its 5' end and to the 7. exon of the gene CG32944 at its 3' end. b) PGC transmigration at stage 5-3 in wt, aen ${ }^{2}$ mutant, genomic aen rescue lines \# 13 and \#14 and nullo-Gal4/UAS-AenA mediated expression of AenA. The PGC transmigration phenoype is fully rescued in line \# 13. In line \# 14 a partial rescue can be observed. The nullo-Gal4/UAS-AenA mediated expression of AenA in the somatic blastoderm results in a partial rescue. $n$ denotes the number of analyzed embryos.

3.2.3 The PGC transmigration phenotype in aen mutants can be rescued by introducing a genomic fragment covering the aen locus

To show that the aen mutation causes the PGC mismigration phenotype observed, rescue experiments were performed. The genomic region of the aen gene including the flanking sequences up to the 5' and 3' neighboring genes (figure 8a) was amplified by PCR from the BAC clone BACR15G02 and cloned into the pCaspeR4 vector (Klemenz et al., 1987) for the generation of transgenic flies by pole plasm injection (Rubin and Spradling, 1982). Two independent transgenic lines carrying the genomic rescue fragment were crossed into the aen ${ }^{2}$ background. In embryos derived from these strains, the ratio of embryos with wt and mutant PGC transmigration phenotypes was examined according to the scheme described in section 3.2.1. The analysis (figure $8 \mathrm{~b}$ ) shows that aen ${ }^{2}$ embryos containing one copy 
of the rescue construct exhibit lower PGC transmigration rates than aen ${ }^{2}$ embryos. $44 \%$ of aen ${ }^{2}$ embryos have strong PGC mismigration phenotype. In the aen genomic rescue experiment this ratio decreases to $6 \%$ using transgenic rescue line $\# 13$ and to $22 \%$ using the independent transgenic rescue line \#14 (wt: 8\%; figure 7c). The complete rescue of the mutant phenotype by the addition of one copy of the aen genomic rescue fragment shows that the observed PGC mismigration is most likely caused by the loss of aen activity.

\subsubsection{The PGC transmigration phenotype in aen mutants can be rescued by Gal4-UAS mediated expression of aenA}

In order to rescue the aen mutant phenotype using an independent technique, the expression of aen gene products was induced in aen mutant embryos utilizing the Gal4-UAS expression system (Brand and Perrimon, 1993). This system consists of the yeast Gal4-transcriptional activator that, via binding to its DNA target sites (UAS, Upstream Activation Sequence), induces the expression of any cDNA cloned 3' of a minimal promoter with the UAS sites. It is a highly established experimental tool to induce the expression of a target gene in Drosophila cells or tissues. In vivo, the temporal and morphological domain of the expression can be chosen by crossing transgenic flies carrying a construct bearing the target gene controlled by a UAS containing promoter region to other fly lines that contain Gal4-driver constructs. These constructs bear the Gal4 gene under the control of a promoter originating from a Drosophila gene. The activity of this endogenous promoter indirectly induces the transcriptional activation of the target gene by activating the transcription of the Gal4 protein (Brand and Perrrimon 1993).

For this purpose, the aenA ORF was cloned into the pUASt vector (Brand and Perrimon, 1993) bearing the minimal promoter with the Gal4 responsive UAS sites. The vector was then utilized for the 
generation of transgenic flies by pole plasm injection (Rubin and Spradling, 1982). Analogous to the genomic rescue, the transgenic lines carrying the UAS-AenA construct and flies carrying the Nullo-Gal4 driver construct were crossed into the $a n^{2}$ background. To induce the expression of aenA in the somatic blastoderm epithelium underlying the PGCs, the aen² mutants bearing the UAS-aenA and Nullo-Gal4 constructs respectively were crossed. The offspring bearing both the UAS and Gal4 constructs was examined following the scheme described in section 3.2.1.

The analysis (figure 8b) shows that the PGC transmigration rate in aen ${ }^{2}$ embryos, in which aenA is expressed under the control of the NulloGal4 driver construct, is lowered as compared to aen ${ }^{2}$ embryos. While $44 \%$ of aen² embryos exhibit a strong PGC mismigration phenotype, this ratio decreases to $34 \%$ in embryos expressing aenA. Even though the rescue efficiency observed in this approach is lower as in the genomic rescue, this result indicates that the activity of the aenA gene product in the somatic cells underlying the PGCs is sufficient for rescuing the PGC mismigration observed in aen² mutants.

\subsection{Expression of aen}

Northern Blot analysis had shown that aen transcripts are present throughout all stages of Drosophila development. Furthermore, the transcripts are highly abundant in the female abdomen and during early embryogenesis (Molitor, 2002). A strong ubiquitous signal of aen transcripts is detectable at stage 1 of embryogenesis (section 1.2; Molitor 2002). By stage 3, when the PGCs are formed at the posterior pole, aen transcripts are included into their cytoplasm. During stage 56 the levels of aen transcript are reduced in the somatic parts of the embryo presumably by specific degradation, whereas the transcripts in the PGCs remain until stage 9. Based on the high transcript levels present in the early embryonic stages and the strong aen expression in 


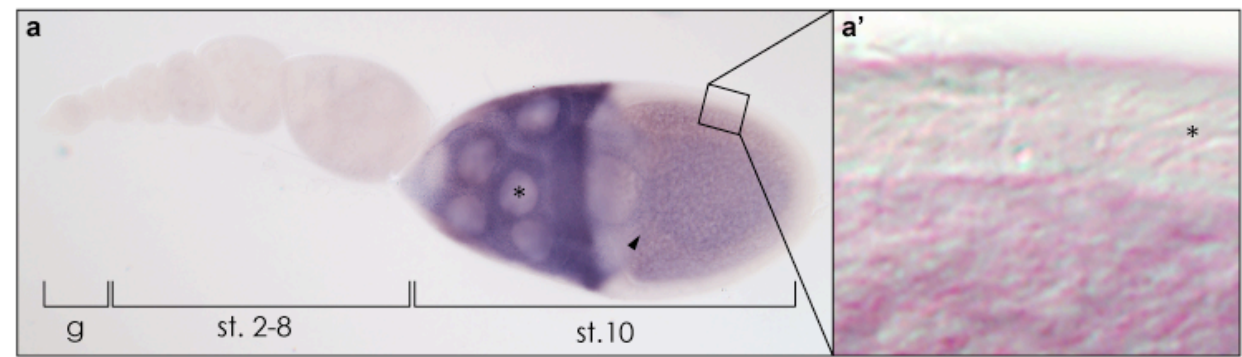

b

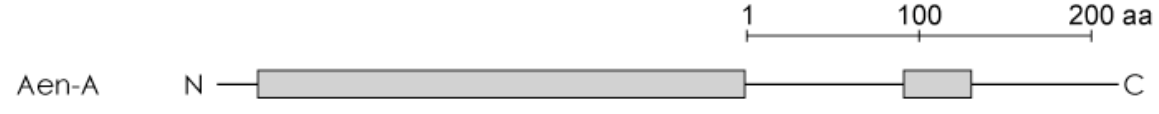

Aen-B

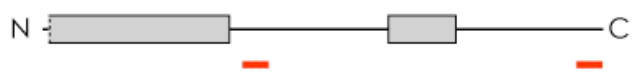

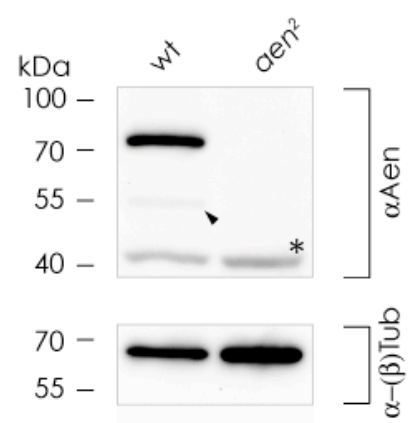

d

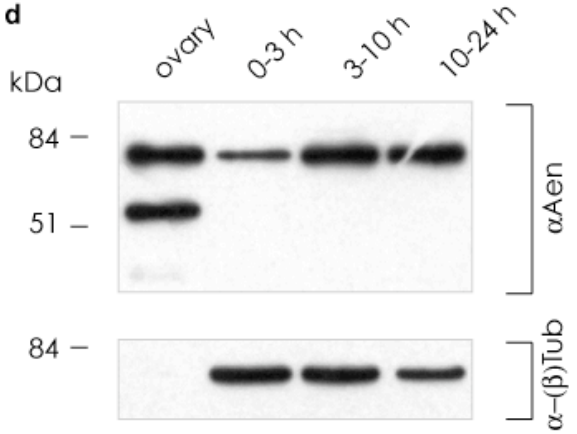

Figure 9: aen expression in development. a) aen in situ of an ovariole with egg follicles of different developmental stages ( $g=$ germarium). aen transcripts cannot be detected in the germarium and oogenesis stages 2-8. At stage 10, aen is strongly expressed in the nurse cells (asterisk) and imported into the oocyte (arrowhead). a') aen is not expressed in the somatic follicle cells (asterisk). b) Sequence position of the peptide-antigens used for the generation of the anti-Aen antibody. Grey boxes represent the conserved $\mathrm{N}$ - and $\mathrm{C}$-terminal domains. c) Upper panel: Western blot detection of the Aen protein in embryo-extracts from $0-22 \mathrm{~h}$ collections with the anti-Aen antibody. The main band in the wt lane corresponds to AenA. A weak band at around 55 kDA height corresponds to AenB. The anti-Aen antibody exhibits little background staining (asterisk). No Aen signal is detected in aen² mutants. Lower panel: loading control with anti-( $\beta$ )Tub. d) Upper panel: Developmental Western blot using the anti-Aen antibody. AenA is translated during oogenesis and embryogenesis. AenB is strongly translated during oogenesis, while its protein levels drop at the beginning of embryogenesis. Lower panel: loading control with anti- $(\beta)$ Tub.

females as revealed by the Northern Blot (Molitor, 2002), aen transcripts are presumably imported into the oocyte as a maternal contribution.

\subsection{1 aen transcripts are restricted to the germline during oogenesis}

To analyze whether the female specific high transcript levels in the Northern Blot reflect only a strong maternal contribution, or whether aen is expressed earlier in oogenesis or in the somatic parts of the egg follicles as well, an in situ hybridization on dissected ovaries was 
performed using a digoxigenin labeled RNA antisense probe detecting both aen transcripts. The RNA in situ staining reveals that aen transcripts are highly abundant in nurse cells and the oocyte after stage 8 (figure 9a). This finding provides a strong indication that aen transcripts are imported from the nurse cells into the oocyte as a maternal load. It also shows that aen gene products are not expressed above the detection level of the RNA in situ during early stages of oogenesis. Additionally aen transcripts are not detected in the somatic follicle cells, surrounding the germline. Together with the fact that homozygous mutant aen ${ }^{2}$ females are viable and fertile it appears highly unlikely, that aen has a major function during oogenesis.

3.3.2 The Aen-antibody specifically detects both Aen protein variants on Western blots

It remained to be tested whether both gene products (figure $5 \mathrm{c}$ ) are transcribed and whether they are equally present at all developmental stages. The two hypothetical transcripts share a nearly identical size of $2695 \mathrm{bp}$ (aenA) and $2542 \mathrm{bp}$ (aenB) excluding the poly-A-tail and can therefore not be clearly distinguished on a developmental Northern-Blot (Molitor, 2002). The aenA transcript features 232 bases not shared with aenB which in turn has 42 specific bases. The high degree of sequence-overlap does not allow for the generation of transcript-variant specific probes.

To overcome this predicament, it was investigated whether the protein variants inferred from conceptual translation of the two postulated transcripts, which differ significantly in size (section 1.2; figure $5 d$ ) are translated in vivo. This analysis was achieved by Western Blot analysis using a specific antibody. Based on the known cDNA sequence two sequences were chosen for peptide synthesis and the immunization of rabbits. The first peptide is located at the native C- 
terminus of both protein variants and the second in a region with a high degree of antigenicity, overlapping between AenA and AenB (figure 9b). This antigen selection was made to ensure that both protein variants are detected by the antiserum produced.

Protein extracts of embryos of wt and aen ${ }^{2}$ were used in Western Blots (figure 9c). The antibody detection resulted in a main band at an apparent molecular weight of approximately $70 \mathrm{kDa}$ and two weak bands with an apparent molecular weight of around 55 and $40 \mathrm{kDa}$ respectively. The comparison of protein extracts from wt and aen ${ }^{2}$ mutant shows that the $70 \mathrm{kDa}$ and $55 \mathrm{kDa}$ bands are exclusively detected in the wt extract, while the $40 \mathrm{kDa}$ band is equally present in both extracts. This finding indicates, that the 70 and $55 \mathrm{kDa}$ bands represent Aen specific signals.

The molecular weight of AenA as deduced from conceptual translation is 59,7 kDA, however analysis of the sequence with the online tool Protein Calculator v3.3 (http://www.scripps.edu/cgibin/cdputnam/protcalc3) reveals that AenA bears a PI of 9,7 , which would result in 8,8 positive charges at $\mathrm{pH} 7,5$, the $\mathrm{pH}$ of the separation gel. The sequence analysis of AenB, with a predicted molecular weight of $37,3 \mathrm{kDa}$, suggests a $\mathrm{Pl}$ of 10,3 resulting in 11,7 positive charges at a pH of 7,5. These additional charges might account for a substantial change in the running behavior of AenA and AenB causing the deviation from the predicted molecular weights to the observed molecular weights of 70 and $55 \mathrm{kDa}$.

The third band with an apparent molecular weight of $40 \mathrm{kDa}$ detectable both in wt and mutant extracts cannot be explained by an partial translation in the aen ${ }^{2}$ mutant. The sequence analysis of the aen gene shows that the largest ORF possibly transcribed from the aen ${ }^{2}$ allele has a length of only 414 bp resulting in a peptide of 13,8 kDa molecular weight. Therefore, this signal is caused by antibody 
crossreactivity with a protein unrelated to AenA and AenB and does not represent an Aen-specific signal.

In the aen² mutant no Aen specific protein is detected. This finding strongly indicates that the aen ${ }^{2}$ mutation is in fact a null allele and that the reduction of the PGC transmigration phenotype in homozygous aen ${ }^{2}$ mutant embryos as compared to aen2/Df[XM3] transheterozygous aen mutant embryos (see section 3.2.1) is due to the acquisition of modifications in the genetic background of the aen ${ }^{2}$ strain.

\subsubsection{AenA and AenB are differentially translated in the course of development}

To characterize the developmental translation profile of AenA and AenB a developmental Western Blot of tissues from wildtype flies was performed. The materials analyzed were protein extracts from dissected female ovaries, embryo collections of 0-3 h, 3-10 h and 10-22 $\mathrm{h}$ in age. The 0-3 h embryo collection covers embryonic stages 1 to 5 from egg laying including the stages of PGC formation as well as blastoderm cellularization. The 3-10 h embryo collection covers the embryonic stages 6 to 13 from the beginning of gastrulation to the end of germband retraction and includes the resident and migratory phase of the PGCs as well as the alignment of the PGCs with the somatic gonadal precursor cells (SGPs). The 10-22 $\mathrm{h}$ embryo collection covers the embryonic stages 14 tol7 from the dorsal closure to embryonic hatching and includes the coalescence of the embryonic gonad consisting of PGCs and SGPs.

The Western Blot (figure 9d) shows that AenA is translated at high levels during oogenesis. This translation is restricted to the late stages of eggfollicle maturation, as inferred from in situ analysis (figure 9a). The AenA levels exhibit a slight reduction in early embryogenesis, increasing 
again after $3 \mathrm{~h}$. This reduction is probably caused by the lower cell number in blastoderm embryos as compared to later developmental stages. In contrast to AenA, AenB is detectable at high levels only during oogenesis, its concentration rapidly decreasing thereafter.

\subsection{The subcellular localization of Aen}

In order to investigate the molecular function of the Aen proteins, it was investigated whether they are specifically localized to subcellular compartments or the cytoskeleton.

\subsubsection{The anti-Aen antibody specifically detects Aen in fixed tissue}

The anti-Aen antibody was tested for applicability in histological stainings of fixed whole mount Drosophila tissues. First, the specificity of the newly generated anti-Aen antiserum was tested. It was administered to a mixture containing aen ${ }^{2}$ mutant embryos and $w t$ embryos expressing Histone2AvD-GFP (Clarkson and Saint, 1999) as a marker to detect specifically the wt embryos. The embryos of both genotypes were combined before dechorionization, fixed and stained jointly. The stained embryos (figure 10a) were analyzed by confocal microscopy. Wt embryos exhibiting Histone2AvD-GFP signal showed a very strong antibody staining in the cytoplasmic space whereas the aen ${ }^{2}$ embryos without the Histone2AvD-GFP signal showed only an extremely weak staining. This result showed that the peptide antiserum is highly specific for Aen and applicable for immunohistological stainings of fixed material.

Based on to the weak expression of AenB during embryogenesis in Western Blots, the signal obtained in embryos stained with the anti-Aen antibody reflects mainly the localization of the AenA protein.

Consequential to the PGC mismigration phenotype first observed during cellularization, the subcellular localization of Aen was 


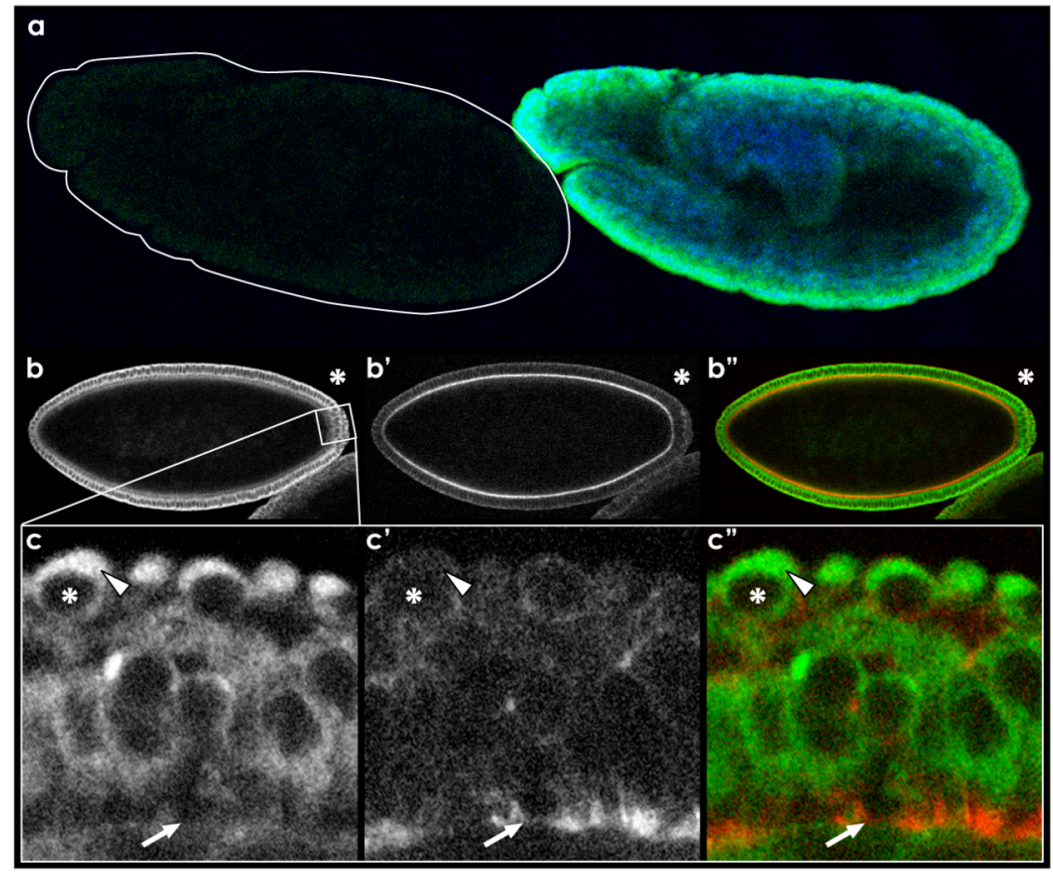

Figure 10: Whole mount antibody staining with the anti-Aen antibody. Immunohistochemical detection of Aen using the anti-Aen antibody in aen ${ }^{2}$ embryos and embryos expressing Histone2AvD-GFP; double immunohistochemical detection of Aen/actin in wt embryos using the anti-Aen antibody and Alexa488-conjugated Phalloidin. a) Test of the specificity of the anti-Aen antibody in whole mount antibody staining of embryos. On the right side, a wt embryo marked with Histone2AvD-GFP (here depicted in blue) is visible, which exhibits strong antibody staining (green). An aen ${ }^{2}$ embryo is located on the left side (outline). The aen ${ }^{2}$ mutant embryo shows only a very weak staining, indicating that the antibody stains very specifically the Aen proteins. b and c) Aen localization during stage 5-3. Left column: Aen; middle column: Actin; right column: overlay. Aen (green), Actin (red). b-b") Cross section of a stage 5-3 embryo. Posterior pole marked with an asterisk. Aen is ubiquitously present in the cytoplasmic regions of the somatic blastoderm and the PGCs but not detectable in the yolky body cavity. c-c") Cross section of the posterior pole with PGCs (asterisks mark nuclei). Forming FC (arrow). The Aen signal exhibits strong focal enrichments in the PGCs (arrowhead).

investigated at embryonic stages 4 and 5. Aen was co-stained with Alexa488-conjugated Phalloidin to visualize the actin cytoskeleton, which is strongly enriched to the cell cortex and the FC. Therefore, the actin cytoskeleton serves as a good marker for the overall morphology of the embryo and for the visualization of the progression of blastoderm cellularization (figures 10 and 11; see also introduction section 1.1.3; fig. 3). Aen can be detected in the PGCs as well as in the somatic parts of the embryo. In the PGCs the Aen signal exhibits strong focal enrichments (figure $10 \mathrm{c}$, arrowhead). This subcellular enrichment remains static in the course of development. In the somatic part of the embryo Aen localization is more dynamic. In pre-blastoderm stages, Aen exhibits ubiquitous cytoplasmic localization but is excluded from 

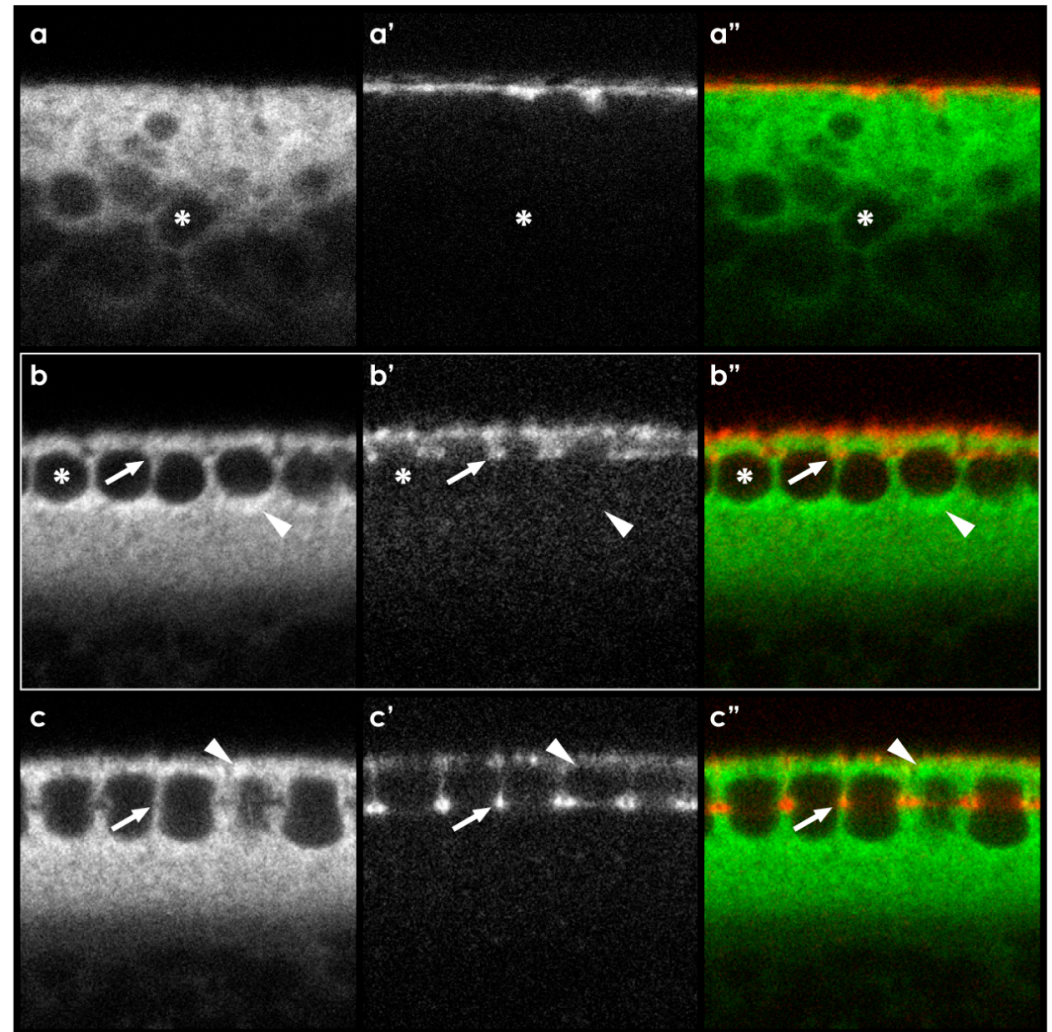

d

d'

d"

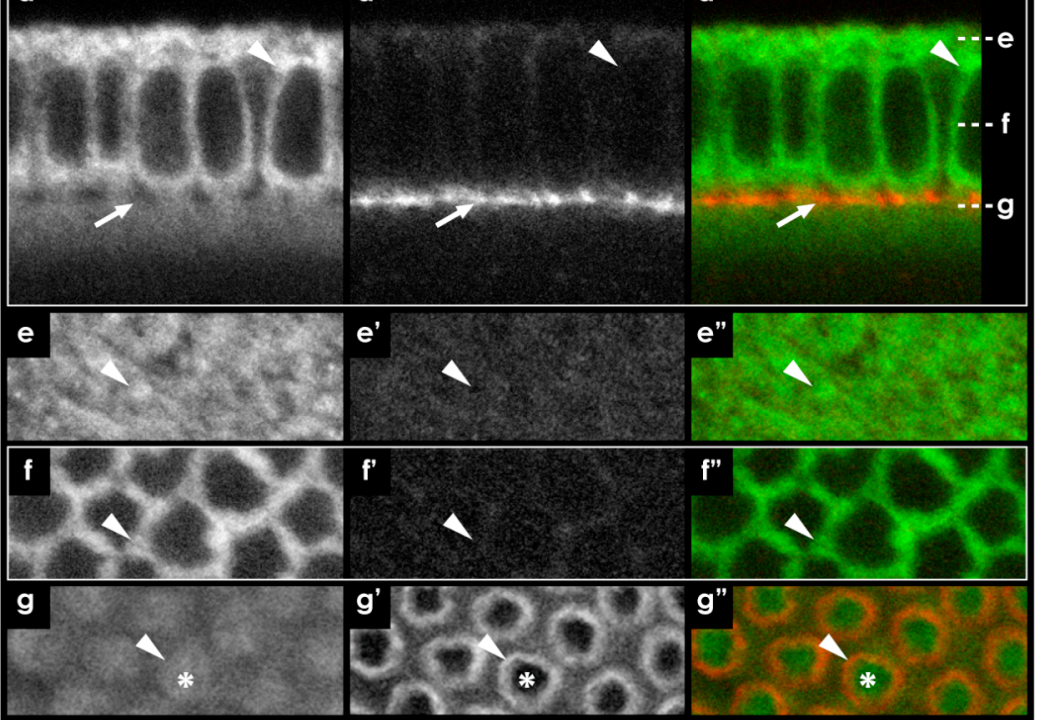

Figure 11: The subcellular localization of Aen during blastoderm cellularization. Double immunohistochemical detection of Aen/actin using the anti-Aen antibody and Alexa488-conjugated Phalloidin. Left column: Aen; middle column: actin; right column: overlay. Aen (green), actin (red). a-a") Cross sections: Stage 3. a) Aen exhibits a cytoplasmic localization and is excluded from yolk particles (asterisk). a') High concentrations of cortical actin. b-b") Stage 5-1: Invagination of the FC (arrow). b) Aen enrichments (white arrowhead) basally of the cortical nuclei (asterisk). b') The actin cytoskeleton is redistributed from the cortex to the FC. C-C") Stage 5-2. c) Aen enrichments (arrowhead) also lateral and apical of the nuclei. c') Concentration of cortical actin is reduced. d-d") Stage 5-3. d) Aen is cytoplasmically distributed with enrichments in the lateral cytoplasm adjacent to the nuclei and the subapical domain. d") The dashed lines illustrate the depths of the grazing sections. e-g) Grazing sections of a stage 5-3 epithelium. e-e") Apical cytoplasm. Aen localization in the apical cytoplasm exhibits focal enrichments (arrowhead). Actin and Aen exhibit no pronounced overlap in distribution. f-f") Half of invagination depth. f) Focal enrichments of Aen in the lateral cytoplasm (arrowhead). f') Actin weakly detected alongside the lateral membranes. g-g") FC. g) Highest Aen concentration in the cytoplasmic stalks (asterisk). Concentration generally lower than in the apical and lateral cytoplasm. g') Actin is organized in contractile rings (arrowhead). 
the yolk and does not show an overlap with cortical actin (figurel la). At the beginning of stage 5, Aen is found in a cytoplasmic region surrounding the nuclear monolayer with enrichments directly apical of the individual nuclei (figurel1b, c). During stage 5-2, the FC is surrounded by Aen even though no direct overlap between the Aen and Actin signal can be detected. From this stage on, Aen enrichments are also found apical of the nuclei. This change in localization continues during the fast phase of cellularization (stage 53) resulting in the enrichment of Aen in the subapical domain and the lateral cytoplasm (figurelld). Grazing sections of a stage 5-3 epithelium (figure 11 e-g) show, that the Aen localization in the apical cytoplasm is irregular and exhibits multiple focal enrichments (figure 11 ), while it is more evenly distributed in the region of the cytoplasmic stalk (figure 11g), adjacent to the FC. The distribution of Aen signal suggests that two pools of the protein exist. One is evenly cytoplasmically distributed, whereas the other co-localizes with subcellular compartments detectable as small granular structures.

In order identify the structures that are enriched with Aen, co-stainings with different markers for structures known to show a similar subcellular distribution and morphology were conducted.

\subsubsection{Aen shows a partial overlap with the recycling endosome marker Rabl 1}

The recycling endosome (RE) is localized in the apical cytoplasm of the somatic blastoderm, directly adjacent to the centrioles of the cortical nuclei. It is a membranous compartment involved in endocytosis and membrane traffick. Its function has been shown to be required for the cellularization of the somatic blastoderm (section 1.1.3; Lecuit et al., 2002; Pelissier et al., 2003). Rab11 is specifically enriched in the RE (Sasamura et al., 1997) but also in small particles, which co-localize also with Golgi markers (Pelissier et al., 2003). 
Confocal analysis of stage 5 embryos co-stained with antibodies against Aen and Rabl 1 shows that no large Rabl1 positive structures are detectable in the PGCs. In the somatic blastoderm however, the Rabl1 antibody marks large structures, characteristic for the RE, directly apical of the cortical nuclei and small particles located in the apical cytoplasm. The overlay of the Rab 11 and Aen signals obtained in the co-staining shows differential results for the RE and the small Rabl1 marked vesicles. Whereas the presumptive REs do not show a high degree of overlap with the Aen signal, the small particles are in most cases Aen and Rabl1 positive (figure 12 a-c). However, many Aen positive structures, especially in the PGCs and the apical portion of the subapical domain do not overlap with Rabl 1.

The finding that Aen only co-localizes with the small Rabl1 positive structures indicates that Aen has no function in the RE itself but might be involved in aspects of membrane traffick during cellularization. The reported presence of Golgi markers in the small Rabll vesicles (Pelessier et al., 2003) would be in accordance with a potential role of Aen in the secretory pathway downstream of the Golgi apparatus.

3.4.3 Aen shows a partial overlap with the Golgi apparatus marker Lava lamp

Based on the observation of Aen signal in small Rabll positive structures, we tested whether a similar overlap could be detected also in structures containing the protein Lava lamp (Lva), which is associated with the Golgi apparatus and derived particles (see section 1.1.3; Sisson et al., 2000; Papoulas et al., 2005). As both, the Aen and the Lva antibody were raised in rabbit, the expression of an AenA-eGFP fusion protein with the Gal4-UAS system (see section 3.2.5) was chosen for co-localization studies. 


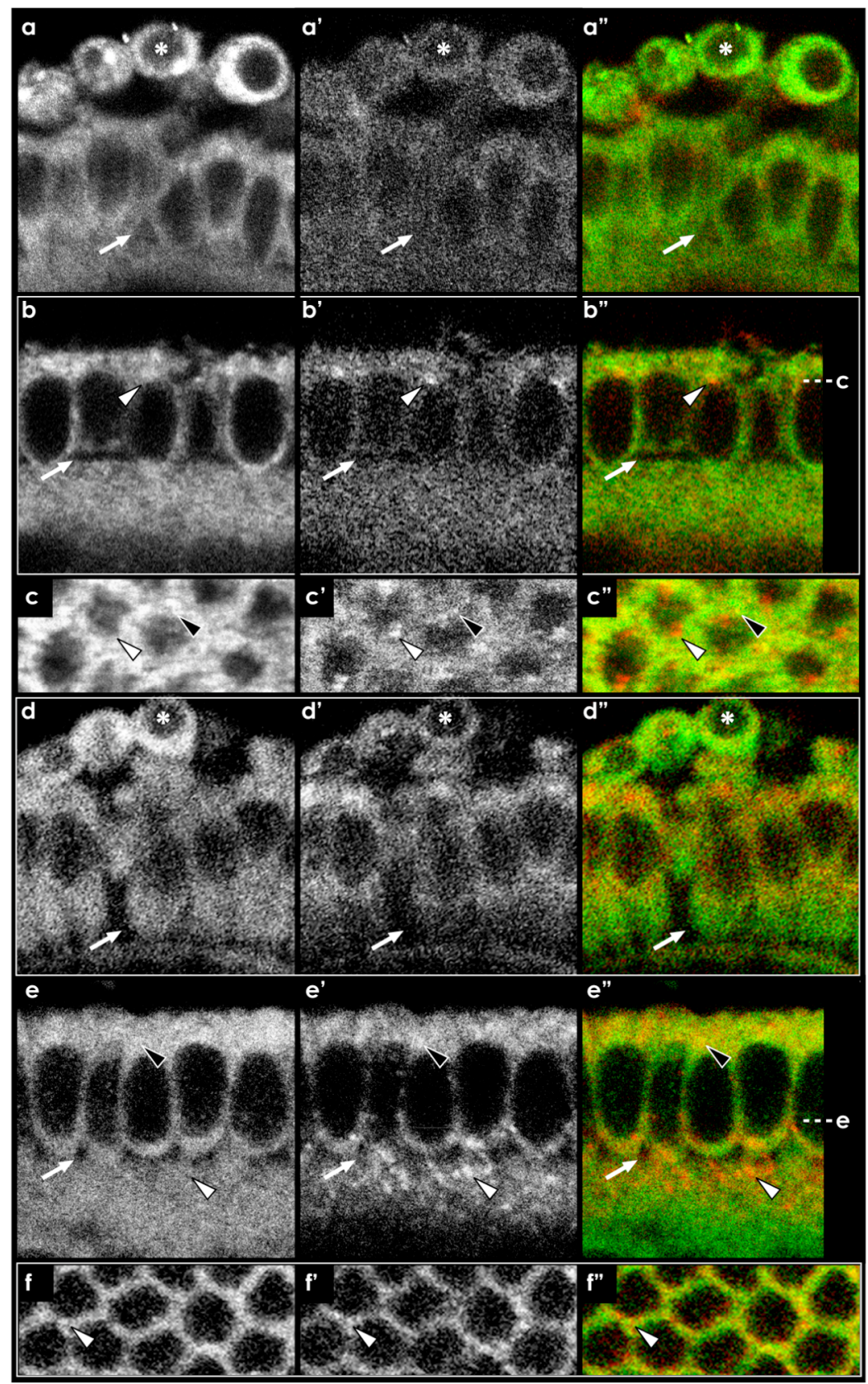

Figure 12: Co-localization of Aen with Recycling Endosome and Golgi. Double immunohistochemical detection of Aen/Rab 11 using anti-Aen and anti-Rabl 1 antibodies. Immunohistochemical detection of Lva in embryos expressing Aen-eGFP using the anti-Lva antibody. $a, b, d, e)$ Cross sections of a stage 5-2 epithelium. Dorsal is up. FC (arrow). a-c) Co-localization of Aen with the Recycling Endosome (RE). RE marked with anti-Rab11. Left column: Aen; middle column: Rab 11; right column: overlay. Aen (green), Rabl 1 (red). a-a") Posterior pole of a stage 5-2 embryo with PGCs (asterisk marks nucleus). While the Aen signal exhibits strong focal enrichments, no Rabll containing particles are detected. (b-b"). Somatic blastoderm at stage 5-2. The Rab11 signal exhibits discrete focal enrichments directly apical of the nuclei (arrowhead). These focal enrichments partially overlap with the structures detected with the anti-Aen antibody. The dashed lines illustrate the depth of the grazing sections. c-c") Grazing section of the apical cytoplasm. The localization of Aen and Rabl 1 shows clear differences in many of the large Rab 11 positive structures (white arrowhead). Small Rabl 1 positive structures (black arrowhead) often show Aen signal. d-f) Co-localization of Aen with the Golgi Aparatus. Golgi marked with anti-lava lamp (Iva). Left column: AenA-eGFP; middle column: Lva; right column: overlay. AenA-eGFP (green), Lva (red). (d-d") Posterior pole of a stage 5-3 embryo with PGCs (asterisk marks nucleus). The strong AenA positive focal enrichments in the PGCs show no overlap with the Lva signals. (e-e"). Somatic blastoderm at stage 5-2. The Lva signal exhibits strong focal enrichments in the basal and lateral cytoplasm. More dispersed signals are detected in the apical cytoplasm. While no enrichment of AenA is detected in the large Lva positive structures (white arrowhead), the two signals overlap in the apical cytoplasm (black arrowhead). f-f") In grazing sections of the basolateral cytoplasm an overlap of AenA and Lva positive structures is visible (white arrowhead). 
Flies containing the UAS construct coding for AenA-eGFP were crossed to a V3-Gal4 driver-line (generous gift from D. St.Johnston) which induces maternal transcription in the $\mathrm{Fl}$ females. The offspring of these females that bear a maternal contribution of AenA-eGFP were then fixed and subjected to a staining with the anti-Lva antibody.

The maternal expression of the AenA-eGFP fusion construct results in a relatively weak eGFP signal (figure $12 \mathrm{~d}$-f). Nevertheless, the AenAeGFP localization appears to be identical to the localization of endogenous Aen under the chosen conditions. The staining with antiLva antibody reveals that differences exist between the localizations of Aen and Lva. The large AenA-eGFP-positive areas detected in the PGCs do not overlap with the Lva-signal. In the somatic blastoderm, Lva is detected in distinct structures in the basal cytoplasm where the AenA-eGFP signal does not exhibit structures of a similar morphology. However in the lateral cytoplasm, an overlap between the Aen and the Lva signal is detected. The Lva detection in these cytoplasmic areas shows smaller areas of signal accumulation, which in many cases are also Aen positive. In the apical cytoplasm, the Lva signal is more dispersed and shows a distribution similar to the AenA-eGFP signal. In summary, Aen is not specifically localized or enriched in the Golgi apparatus. Nevertheless it seems to co-localize with a subset of smaller structures containing the Golgi marker Lva.

\subsubsection{Aen partially co-localizes with the Microtubules}

Based on the observation that Aen is found in particles potentially associated with the secretory pathway and that it is additionally present at high concentrations in the lateral cytoplasm and the subapical domain, we tested whether Aen positive structures colocalize with the Microtubule (MT) network. During the formation of the polarized epithelial cells of the blastoderm, MT have an important function in the transport of vesicles and organelles such as the lipid 




Figure 13: Localization of Tubulin and Aen. Double immunohistochemical detection of Aen/( $\beta)$ Tubulin using anti-Aen and anti-( $\beta$ )Tubulin antibodies. Left column: Aen; middle column: ( $\beta$ )Tubulin; right column: overlay. Aen (green), ( $\beta$ )Tubulin (red). a-c) Cross sections of Stage 5-2. a-a") Overview. The asterisk marks the dorsoposterior side of the embryo. b-b") Posterior pole with PGC (asterisk marks nucleus). b) The PGCs exhibit strong Aen signals, often focally enriched. b') Adjacent to the PGC nulceus the centriole is detected as a singular Tubulin rich structure. C-c") Somatic blastoderm. Aen and Tub exhibit strong signals in the apical cytoplasm, extending in between the nuclei towards the FC. C") Enrichments of Aen and Tubulin partially overlap in the apical and lateral cytoplasm (arrowheads). No enrichments of Aen and Tubulin can be found in the FC (arrow). The dashed lines illustrate the approximate depth of the grazing sections. d-e) Grazing sections. d-d") apical cytoplasm of the lateral blastoderm. d) The cytoplasmic distribution of Aen shows local enrichments. d') Also Tubulin is unevenly distributed. Focal enrichments of Tubulin thereby might represent centrioles. d") The Aen and Tubulin signals exhibit an overlap in part of the focal enrichments detected (arrowhead). In addition structures with high concentrations of Aeneas but no enrichment in Tubulin occur. e-e") Lateral cytoplasm. e) The cytoplasmic distribution of Aen shows local enrichments (arrowhead). e') Vertical Tubulin bundles in the cytoplasm and lining the nuclei appear as punctate structures in the grazing section. e") The Aen and Tub signals exhibit an overlap in part of these punctae.

droplets (LD) (Welte et al., 1998; reviewed in Jäckle and Jahn, 1998). MT bundles extend throughout the cytoplasm vertically to the epithelial surface with the minus-ends directed to the cortex and the plus-ends directed to the yolk (reviewed in Welte, 2004). To investigate if Aen co-localizes with MT, antibody stainings using anti-Aen and anti$(\beta)$ Tubulin were carried out with embryo collections of early stages (figure 13). The MT bundles appear as thick filamentous structures stretching from the apical moiety of the cytoplasm to the 
cellularization front. The cortical part of the MT network appears as focally condensed structures in the apical cytoplasm. The grazing section (figure 13 e) shows that MT bundles are arranged directly adjacent to the nuclei.

The overlay of the $(\beta)$ Tubulin and Aen signals obtained in the costaining shows that the Aen signal partially co-localizes with the Tubulin signal. In cross sections the Aen signal is detected next to the nuclei and the FC, this localization to a great extend reflects the Tubulin localization in filamentous structures found next to the nuclei and the cellularization front. Furthermore, the structures in the apical region of the cells show a partial overlap between Aen and $(\beta)$ Tubulin. The Aen signal thereby extends further towards the embryonic cortex. In optical sections parallel to the surface of the embryo, the ( $\beta$ )Tubulin and Aen signals show a partial overlap in the apical cytoplasm while in the lateral cytoplasm small, punctate enrichments of Aen are detected that overlap or are directly adjacent to the Tubulin bundles. These findings are in agreement with the observation that Aen colocalizes with particles that are potentially part of the secretory pathway.

\subsection{Biochemical interaction studies}

As a means to gain further insight into the molecular function of the Aen protein, its ability to bind other, characterized factors was investigated. The basis for candidate selection was the analysis of the aen phenotype, the subcellular localization of the Aen protein and published yeast two-hybrid data from a genome-wide screen (Giot et al., 2003).

The analysis of the aen phenotype had shown that the earliest timepoint of PGC mismigration was at embryonic stage 5-2, when the somatic blastoderm cellularizes. The coincidence of the PGC 
mismigration and the cellularization of the somatic PMG cells raised the question which cellular process depends on aen activity: the transmigration activity of the PGCs or the cellularization process preventing the PGC mismigration normally at this stage of development. In the literature only one factor, Slow as molasses (Slam) is described as being required both for cellularization of the somatic cells and for the migration of PGCs in later stages (see section 1.1.4; Stein et al., 2002; Lecuit et al., 2002). Furthermore, the Slam protein is expressed exclusively at the time of cellularization. Therefore, slam appeared to be a good candidate for an interaction partner of Aen.

Shotgun (Shg; Drosophila E-Cadherin; see section 1.1.3; Oda et al., 1994; Tepass et al., 1996) is an important component of the basal and apical adhesive junctions formed during blastoderm cellularization. Mutations of shg affect the adhesion between the somatic blastoderm cells (Tepass et al., 1996). Therefore Shg was tested for biochemical interaction with Aen.

In addition the factor Actin related protein 1 (Arp1) (Fryrberg et al., 1994) was tested, which had been identified as a potential interactor of Aen in a genome wide yeast two-hybrid approach (Giot et al., 2003). Arpl is a component of the dynactin complex mediating MT based transport.

\subsubsection{In vitro interaction studies of Aen with Arp 1, Slam and Shg}

The method chosen for initial analysis was a pulldown-approach utilizing tagged-Aen protein to bind in vitro translated target proteins labeled with ${ }^{35 S}$-cysteine and -methionine. Several constructs coding for Aen fusion-proteins with solubility promoting tags (reviewed in Esposito and Chatterjee, 2006) were generated. The bacterial expression levels and solubility of Maltose Binding Protein-AenA, Thioredoxin-AenA, N-utilizing substance-AenA and Glutathione-S- 
c

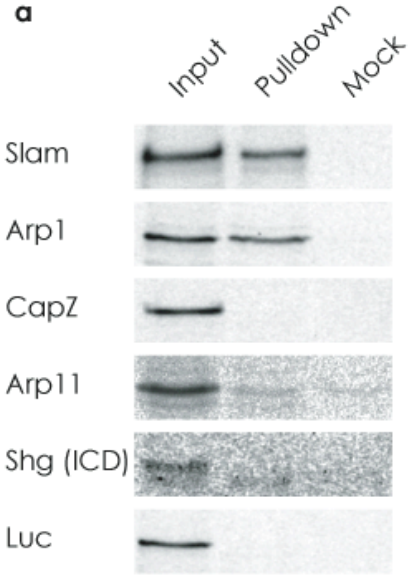

b

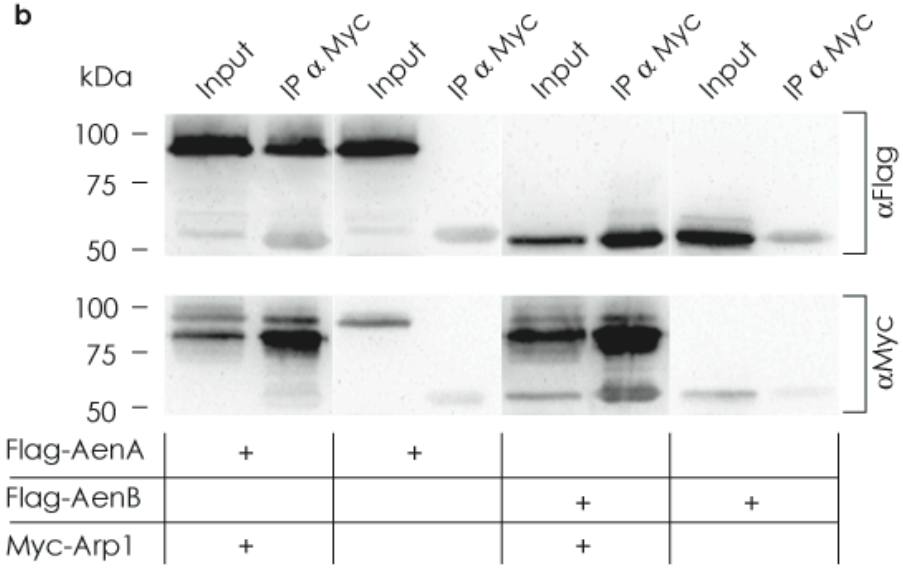

c

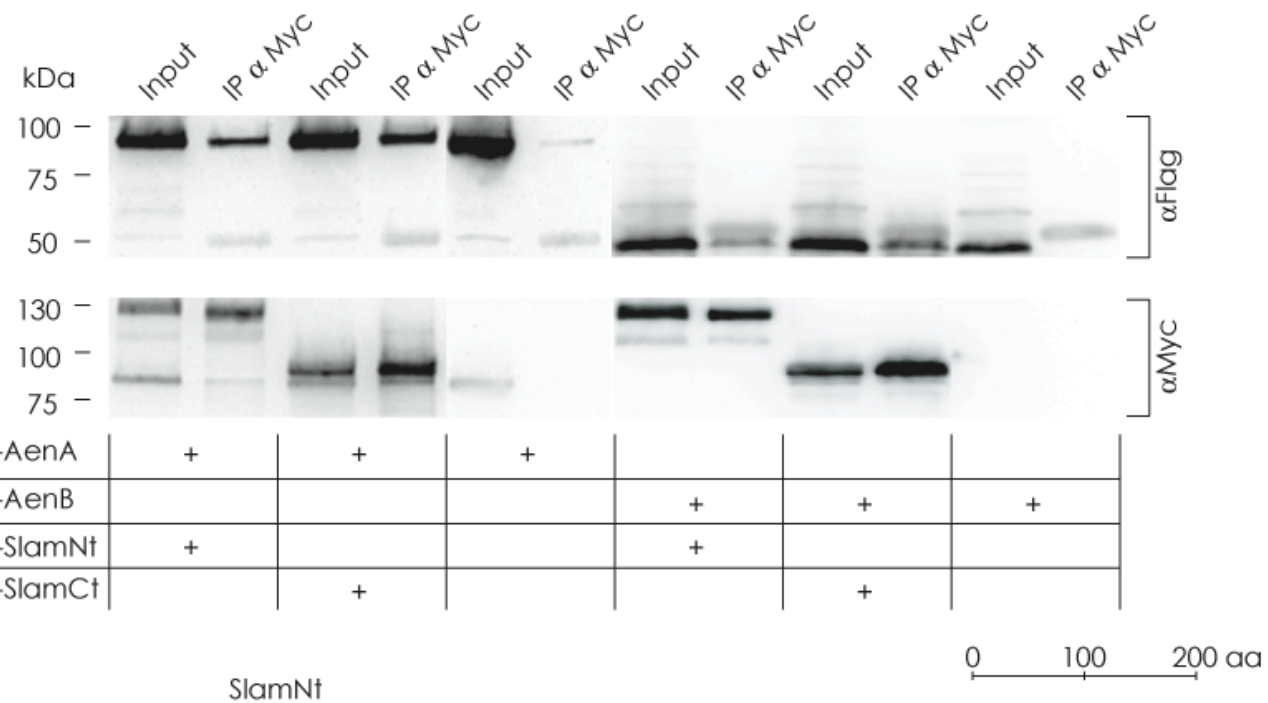

d

Figure 14: Biochemical interaction studies with potential interactors of Aen. a) GST-Pulldowns of in vitro translated proteins. Left column: Input of in vitro translated protein; middle column: pulldown using GST-AenA coupled to beads; right column: mock pulldown using GST coupled to beads. The in vitro translated proteins are indicated on the left. Slam and Arpl bind specifically to AenA (top two rows in the middle column). b) Co-Immunoprecipitation of Flag-tagged Aen variants with Myc-tagged Arpl. Upper panel: Western blot detection with anti-Flag. Medium panel: Western blot detection with anti-Myc. Lower panel: Transfection scheme of Kc167-cells. AenA and is specifically co-precipitated with Arpl (upper panel, second lane). AenB and is specifically co-precipitated with Arpl as well (upper panel, sixth lane). C) Co-Immunoprecipitation of Flag tagged Aen variants with truncated Myc-tagged Slam proteins. Upper panel: Western blot detection with anti-Flag. Medium panel: Western blot detection with anti-Myc. Lower panel: Transfection scheme of Kcl67-cells. AenA and is specifically co-precipitated with the Slam-N-terminal fragment and the Slam-C-terminal fragment (upper panel, second and fourth lane). AenB and is specifically Co-precipitated with the Slam-N-terminal fragment and the Slam-C-terminal fragment (upper panel, ninth and eleventh lane). d) Coverage of the truncated, Myc-tagged Slam-N-terminal fragment and Slam-C-terminal fragment (blue; dark grey = representation of the full length Slam protein).

Transferase-AenA (GST-AenA) were tested. In these tests only the GSTAenA fusion exhibited expression rates and solubility sufficient for medium scale expression and purification. 
The Slam protein as well as the protein Luciferase, used as an internal negative control in this experiment, were generated in a combined in vitro transcription/translation reaction (section 2.3.8) and applied on a resin loaded with GST-AenA as well as mock resin loaded with GST without fusion portion. After extensive washing, the proteins bound to the resin were subjected to SDS-PAGE and exposed to image-plates. The analysis (figure 14a) showed that the Slam protein binds to the resin preloaded with Gst-AenA while it does not bind to the mock-resin preloaded with GST. In contrast, Luciferase used as a control shows no interaction with the GST-AenA loaded and the mock resin. This result gave a first indication that a specific interaction might exist between Slam and AenA.

Based on the cytoplasmic localization of Aen an interaction of Aen with the extracellular and transmembrane domains of Shg could be excluded. Therefore, only the intracellular domain of Shg was tested for a potential interaction with AenA. In order to express the cytoplasmic tail, the sequences encoding the extracellular and transmembrane region were removed by PCR and an artificial start codon was introduced into the cDNA. The in vitro translation resulted in a protein with the expected size of $16 \mathrm{kDa}$ as deduced from the aminoacid sequence of the cytoplasmic tail of Shg. Even though the protein amount obtained in vitro was comparably low, the results show that no direct interaction exists between the intracellular domain of Shg and GST-AenA (figure 14a).

To investigate if Arpl is able to bind to purified recombinant AenA, it was labeled and subjected to the GST-pulldown assay as described above. The result of the analysis showed that Arpl binds to GST-AenA loaded resin and does not bind to the mock-resin (figure 14a). To further characterize the interaction between AenA and Arpl, the proteins CapZ and Arp 11 were tested in the GST-Pulldown analysis as 
well. Both proteins are subunits of the dynactin complex and direct binding partners of the Arpl protein (reviewed in Schroer 2004, Imai et al., 2006). The results of the pulldown assay show, that neither Capz nor Arpll exhibit specific binding affinity to AenA-GST. These findings, which are in good agreement with the results of the yeast two-hybrid approach (Giot et al., 2003), indicate that AenA might be associated to Arpl as a part of the dynactin complex.

In summary, the GST-pulldown experiments revealed an interaction of GST-AenA with in vitro translated Slam and Arpl, but no interaction with Capz, Arp1 1, the cytoplasmic tail of Shotgun or the control protein Luciferase. To confirm the obtained interactions of AenA with Slam and Arpl, independent protein-protein interaction experiments were performed.

\subsubsection{AenA and AenB interact with Arpl and Slam in co- immunoprecipitation experiments}

The in vitro data provided initial evidence for the interaction between Aen and Arpl. In order to achieve an independent control of these experimental results, co-immunoprecipitation experiments were conducted. To allow for an efficient immunoprecipitation and detection of the proteins in the subsequent western blot, AenA, Slam and Arpl were differentially tagged. Vectors were generated for the expression of 3xFlag-Aen and 10xMyc-Arpl, tagged at their $\mathrm{N}$-termini, under the control of an Actin promoter. Drosophila Kcl67-cells (Cherbas et al., 1977) were transfected and harvested after 4 days. An antibody against the Myc-Tag was used for immunoprecipitation; Western Blot detection of co-precipitated proteins was carried out using an anti-Flag antibody.

The results of the Co-IP (figure 14b) show that the Flag-AenA protein is co-precipitated with the Myc-Arpl protein. Thus the interaction of Aen 
with Arpl could be shown by GST-pulldown experiments and in coimmunoprecipitation and are in good agreement with the results of the yeast two-hybrid experiments (Giot et al., 2003).

To restrict the region of Aen required for the interaction with Arpl, the interaction of Arpl with the N-terminally shortened Aen variant AenB was analyzed in co-immunoprecipitation experiments as well (figure 14b). Since AenB interacts with Arpl in a comparable strength the interaction domain could be restricted to the region between aa 207 and 546 of AenA.

The interaction between Aen and Slam, which had been shown in the in vitro pulldown assay, was likewise tested by means of coimmunoprecipitation of tagged version of full length AenA and Slam. The expression strength of a 10xMyc-fusion of full length Slam, a protein of $1291 \mathrm{ad}$, was found insufficient for co-immunoprecipitation experiments. Therefore, two constructs coding for truncated Slamversions representing the $\mathrm{N}$-terminal 834 aa and the C-terminal 493 aa of the protein both tagged with a 10xMyc-tag were generated (figure $14 d)$.

The results of these experiments are displayed in figure 14. AenA shows interaction with the $\mathrm{N}$ - and $\mathrm{C}$-terminal part of Slam, indicating that either the Slam protein contains two regions interacting with AenA or that the interacting domain extends over the region at the boundary between the two Slam fragments expressed.

To restrict the interacting region of Aen the AenB protein was tested for binding to the $\mathrm{N}$ - and $\mathrm{C}$-terminal truncated Slam variants as described for AenA. The experiment revealed an identical interaction of AenB with two Slam variants as shown for AenA, indicating that the Slam interacting region of Aen must be located in the overlapping 

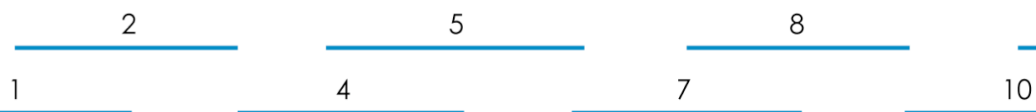

8

11

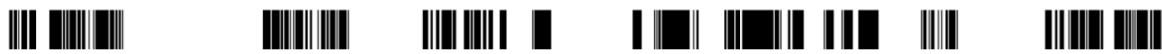

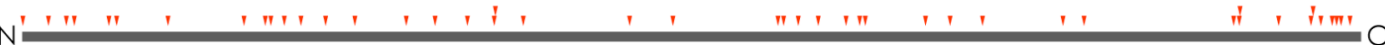

b

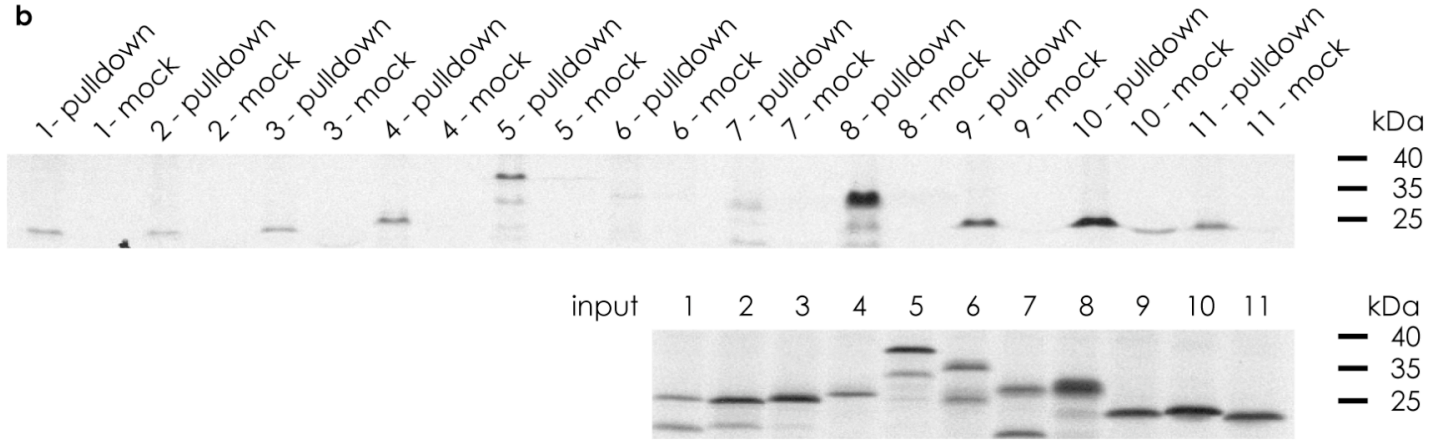

Figure 15: Aen interacting domain of Slam. a) Map of the Slam protein. The coverage of 11 fragments of the Slam protein is represented in blue. Vertical lines mark residues conserved between D. melanogaster, $D$. simulans, D. yakuba, D. erecta, D. perisimilis, D. sechellia, D. mojavensis, D. pseudoobscura, D. virilis, D. ananassae and D. grimshawi as determined with the evoprinter tool. Red triangles mark methionine and cysteine residues bearing ${ }^{35}$ S-labelling in in vitro translation experiments. b) Pulldown of Slam fragments. Upper panel: Pulldown using GST-AenA coupled to beads. Mock pulldown using Gst coupled to beads. Lower panel: input of protein generated by in vitro translation.

sequence between both Aen variants, mediating the interaction with at least two regions of the Slam protein.

\subsubsection{Mapping of the Aen interacting domains of Slam}

The co-immunoprecipitation experiments with Slam, AenA and AenB had revealed that both the $\mathrm{N}$ - and the C-terminal regions of Slam interact with Aen. To restrict these domains further, a series of 11 overlapping Slam fragments were cloned (figure 15a), in vitro translated and tested for interaction with AenA using the GSTpulldown approach described in section 3.5.1. The fragments had an average length of about 200 aa covering the entire sequence of the Slam protein. Such a systematic approach was required as no protein domains are identified in the Slam protein and no data are available that help to refer the biological functions of Slam to specific regions of the protein (Stein et al., 2002; Lecuit et al., 2002; SMART). The 
systematic approach reveals that two regions of Slam interact with GST-AenA in vitro. A strong interaction is detected in a region located in the C-terminal half covering the amino acid domain ranging from aa 705 to aa 1106, represented by the fragments 7,8 and 9 , and a weaker one in the $\mathrm{N}$-terminal half a region ranging from a 300 to aa 612, represented by the fragments 4 and 5 . So both the coimmunoprecipitation experiments and the GST-pulldown experiment identified two domains of Slam, which interact with Aen.

\subsection{Co-localization of Aen with its interaction partners}

The biochemical interaction assays had shown, that Aen binds Arp 1 and Slam both in in vitro Gst-pulldown assays and in vivo as shown by co-immunoprecipitation experiments after transient transfection of cultured cells. To determine whether the interactions of Aen with Arp 1 and Slam could occur in vivo in the PGCs or the somatic blastoderm and could therefore be of biological relevance, a series of double stainings was conducted. A co-localization of Aen with its potential interacting partners would provide an important indication for the biological relevance of the interactions.

\subsubsection{Aen co-localizes with Dynein Heavy Chain}

Since no specific antiserum against Drosophila Arp87 is available, the subcellular localization of Arpl was determined indirectly. For this purpose, an antibody detecting Dynein Heavy Chain (Dhc), the motor protein which acts in conjunction with the dynactin complex (section 1.1.1; reviewed in Schroer 2004) was applied in double stainings with anti-Aen. A high degree of overlap between Aen and Dhc exists in the somatic blastoderm (figure 16a-d). However, in the PGCs no accumulations of Dhc analogous to the strong focal enrichments of Aen are observed. In cross sections of somatic cells of a stage 5-1 embryo, the enrichments of the Aen staining basally of the nuclei (see 
also figure 11) are also observed for Dhc (figure 16a, arrowhead). At stage 5-3, the focal enrichments in the apical cytoplasm and the basal cytoplasmic distributions show a strong overlap of Aen and Dhc (figure 16c). Minor differences in localization are visible in the region of the FC where the Dhc concentration appears to be elevated in respect to Aen. The co-localization of Aen and Dhc provides a strong indication that Aen and Arpl, which belongs to the same protein complex as Dhc, co-localize as well.

\subsubsection{Aen co-localizes partially with Slam}

Based on the fact that both Aen and Slam antibodies were generated in rabbits, it was necessary to use a Slam-HA fusion protein (Lecuit et al., 2002) that was expressed in early embryos using the maternal V3GAL4 driver line (section 3.2.5). The Slam fusion protein is localized identical to the endogenous Slam protein (Lecuit et al., 2002, data not shown).

The signals obtained in the antibody staining of fixed embryos show that Aen and Slam-HA, even though exhibiting differences in their apicobasal distribution, overlap in structures with high Slam-HA content. The Slam-HA protein is strongly enriched in the FC and the basolateral membrane adjacent to it and shows only a minor cytoplasmic signal. In contrast, the highest Aen concentrations are detected in the apical cytoplasm. Optical sections of the basolateral membrane and the FC, that runs parallel to the embryonic surface, show that both proteins, Aen and Slam-HA, exhibit overlapping enrichments in the lateral cytoplasm, adjacent to the basolateral membrane and the FC. Aen is not excluded from the Slam-HA positive structure marking the leading front of the FC, however the highest concentrations of Aen are found in the cytoplasmic stalks in regions directly adjacent to areas with strong Slam signal. 

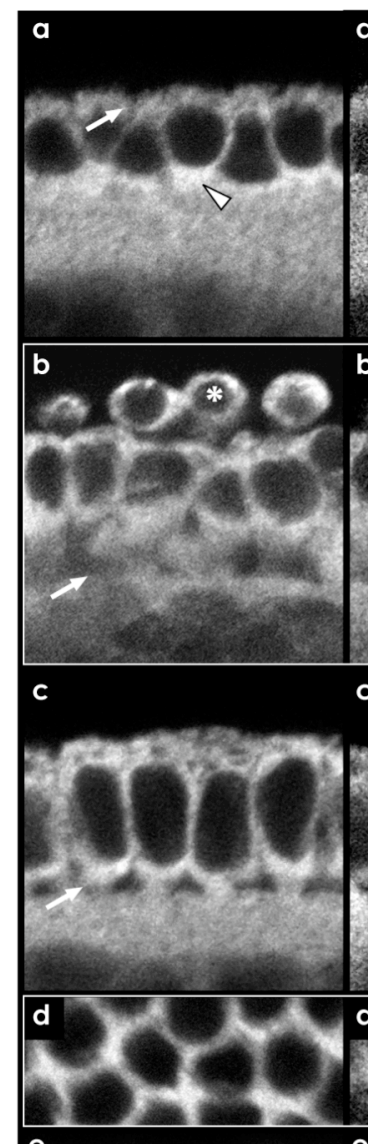

e a'

$\mathbf{a}^{n}$
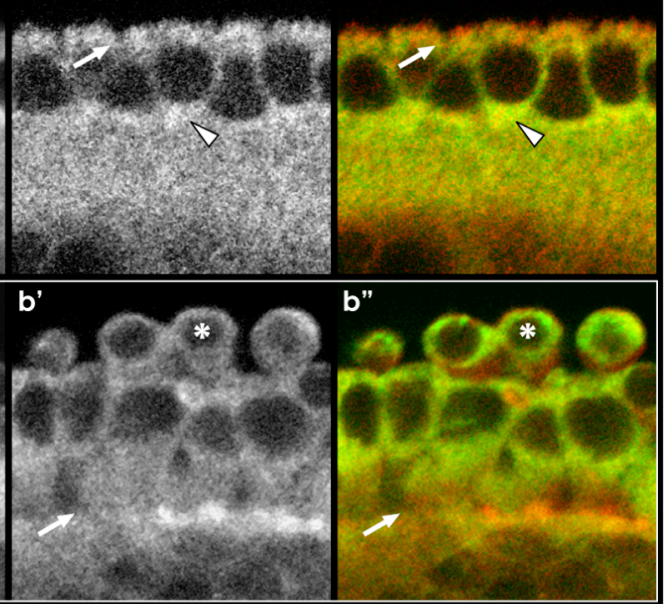

$\mathbf{c}^{\prime}$

c"
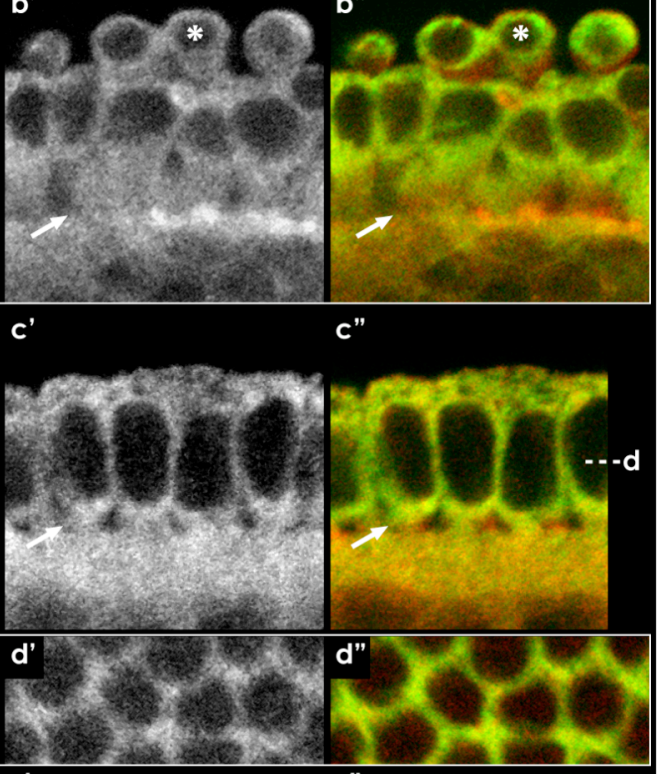

e"

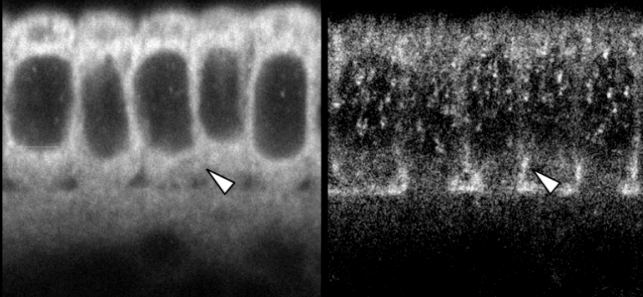

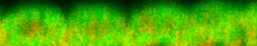

1



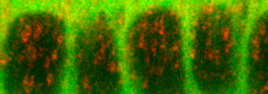
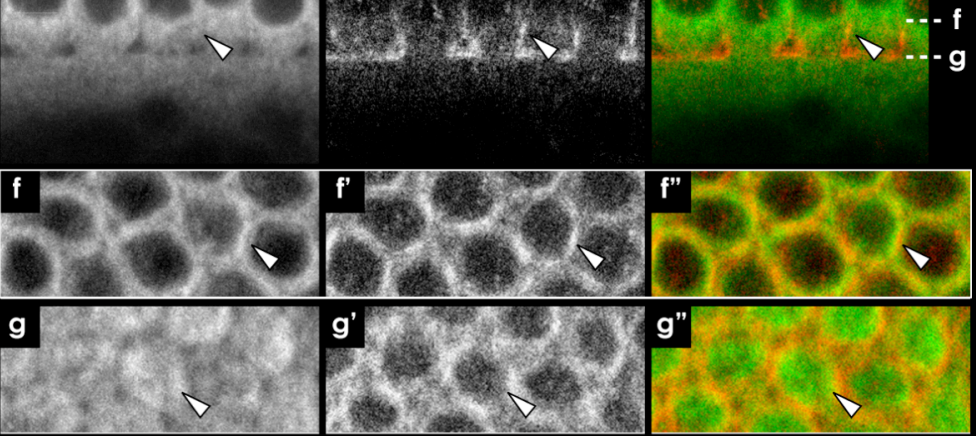

Figure 16: Colocalization of Aen with interacting partners. Double immunohistochemical detection of Aen/Dynein heavy chain (Dhc) and Aen/Slam-HA using anti-Aen, anti-Dhc and anti-HA antibodies. a-d) Co-localization of Aen with Dhc, a main component of the dynein-dynactin transport complex. Left column: Aen; middle column: Dhc; right column: overlay. Aen (green), Dhc (red). a-a") Cross section of a stage 5-1 epithelium. Forming FC (arrow). The enrichment basally of the nuclei detected in the Aen staining is also observed for Dhc. In addition, Dhc exhibits also apical enrichments. b-b") Posterior pole of a stage 5-2 embryo with PGCs (asterisk marks nucleus). While the Aen signal exhibits strong focal enrichments, the Dhc signal in the PGCs is only very weak. Only a minority of the Aen enrichments bears a corresponding Dhc signal. c-c") Somatic blastoderm at stage 5-3. The focal enrichments in the apical cytoplasm and the basal cytoplasmic distributions show a strong overlap of Aen and Dhc. Minor differences in localization are visible in the region of the FC (arrow) where the Dhc concentration appears to be elevated in respect to Aen. The dashed lines illustrate the depth of the grazing sections. d-d") Grazing section of the lateral cytoplasm. The localization of Aen and Dhc exhibits a high degree of overlap. e-g) Co-localization of Aen with Slam-HA. Left column: Aen; middle column: Slam-HA; right column: overlay. Aen (green), Slam-HA (red). e) The 
apicobasal distribution of Aen and Slam-HA exhibits clear differences. While the Aen concentration is highest in the apical cytoplasm, Slam-HA is detected at high concentrations in the FC. However an overlap of the signal is detected on the subcellular level (arrowhead). f) Aen and Slam-HA show overlapping enrichments in the lateral cytoplasm, adjacent to the basolateral membrane and the FC (arrowhead). g) Aen is not excluded from the Slam-HA positive structure marking the leading front of the FC, however the highest concentrations of Aen are found in the cytoplasmic stalks in regions directly adjacent to areas with strong Slam signal (arrowhead).

The differences in apicobasal distribution of Aen and Slam-HA do not support the results of the biochemical interaction studies, however the partial overlap found in the basolateral membrane and the FC indicates that a subpopulation of Aen can interact with Slam in vivo.

\subsection{Genetic interaction studies of aen with slam}

To investigate whether the biochemical interaction and colocalization of Aen and Slam reflect a biologically relevant interaction, changes in cellularization velocity of aen mutant embryos were assayed. The genetic interaction of aen and slam during the cellularization of the somatic blastoderm cells was tested by analysis of the blastoderm morphology. As a third assay, the occurrence of cuticle phenotypes later in development was analyzed.

\subsubsection{Genetic interaction of aen and slam during cellularization}

Earlier studies of slam (Stein et al., 2002) had shown that zygotic null mutant embryos that still bear half of the normal maternal contribution of slam mRNA exhibit only mild developmental defects characterized by PGC mismigration from stage 10 onwards (see section 1.1.4). When additionally the maternal contribution of slam mRNA was removed, it was found that the resulting maternal-zygotic null mutant embryos exhibited strong defects in blastoderm cellularization during stage 5, resulting in a preterm stop of FC invagination and extensive cell death in the incompletely cellularized epithelium. It was found that the localization of the proteins D-PATJ (section 1.1.3; Bhat et al., 1999; Pielage et al., 2003), a component of the FC, and Neurotactin (Nrt; section 1.1.3; Hortsch et al., 1990), an adhesion factor localized to the 
apicolateral membranes, is altered in the affected epithelia (Stein et al., 2002; Lecuit et al., 2002). While D-PATJ positive membrane domains broadened and fuzzied, Nrt, in addition to its enrichment in the apicolateral membranes, is found ectopically enriched in the apical cytoplasm (Lecuit et al., 2002). This localization defect might be the consequence of an impairment of vesicle-integration into the apicolateral domain of the plasma membrane (PM) (Lecuit et al., 2002).

To characterize a potential genetic interaction between aen and slam it was analyzed if the loss of aen activity increases the strength of the zygotic slam mutant phenotype. The rationale of this experiment was that the absence of Aen would reduce the remaining zygotic Slam activity thereby leading to the occurrence of cellularization defects even in the presence of maternal slam mRNA (figure 18) if the potential interaction between Aen and Slam is important for slam activity. For this purpose, the aen ${ }^{2}$ mutation was crossed into the genetic background of both the slam' mutation and the slam deficiency (Df(2L)Exel6016, covering cytogenetic bands 26C1-D1). Crossing of these fly stocks resulted in offspring, bearing half of the maternal contribution (for details see Materials and Methods section 2.2.3). As a control, the cellularization in slam single mutant embryos (for details see Materials and Methods section 2.2.3), and aen² embryos was investigated. For the analysis, embryos of wt, the aen ${ }^{2}$ and slam single mutants and slam-aen double mutants were fixed and stained using antibodies detecting the D-PATJ and Nrt, which both are good markers for the morphology of the forming epithelium and in addition might exhibit localization defects as shown in the maternal zygotic slam mutant (Stein et al., 2002; Lecuit et al., 2002). 

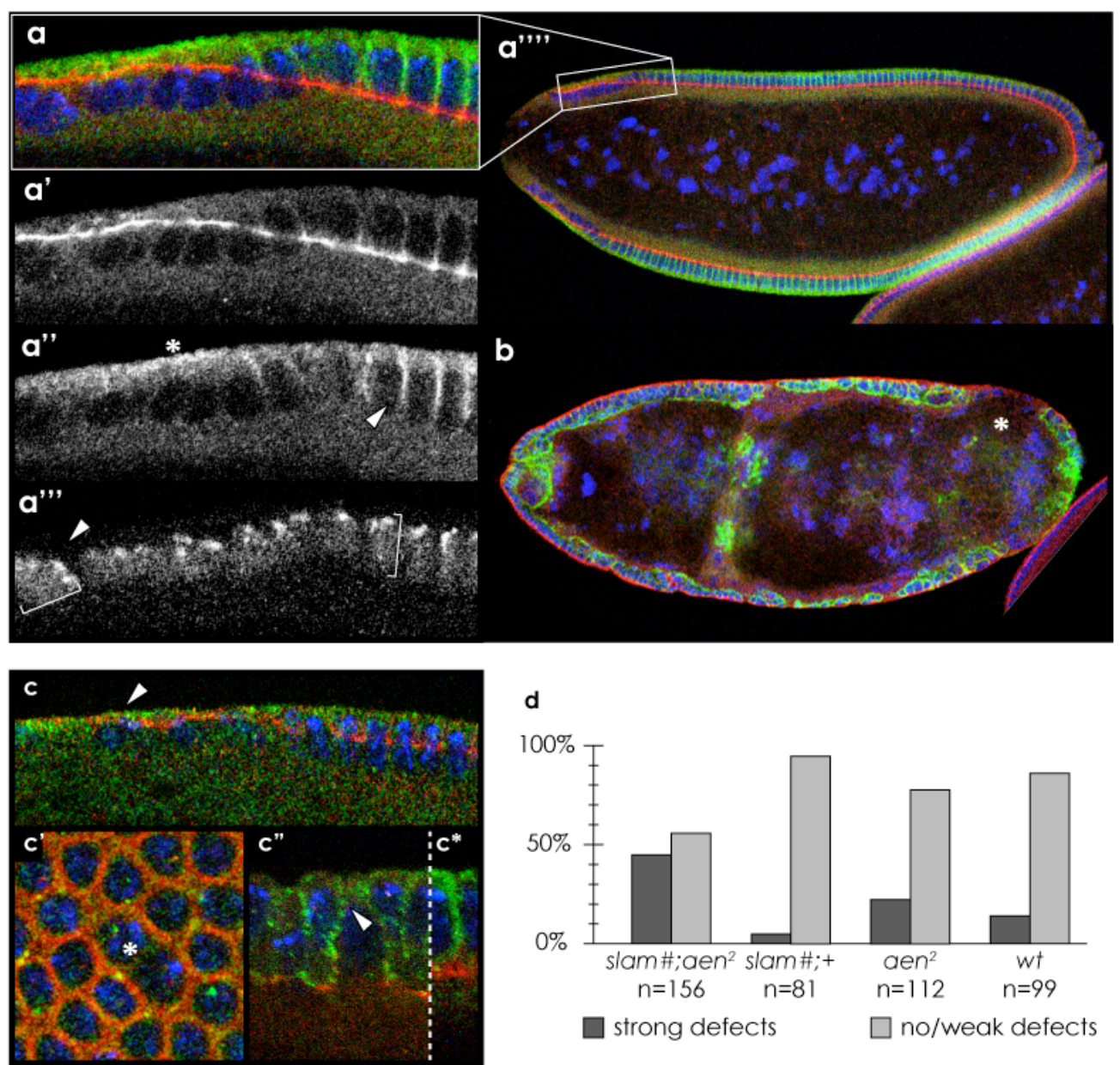

Figure 17: Genetic interaction of aen and slam. Triple immunohistochemical detection of Nrt/PatJ/DNA using anti-Nrt, anti-PATJ antibodies and DRAQ5. a and b) Cellularization phenotypes in aen-slam double mutants. a) Cellularization failure at the embryonic poles. red: Dlt; green: Nrt; blue: DNA. a') Dlt marks the FC. The depth of FC progression is strongly reduced towards the embryonic pole (in left direction) as compared to the middle of the embryo. a") Nrt is integrated into the lateral membranes during membrane invagination (arrowhead). In the regions in which cellularization is affected, Nrt accumulates in the apical cytoplasm (asterisk). a"') the nuclei exhibit uniform length (brackets) and morphoogy in intact and affected regions. In affected regions, single nuclei detach from the embryonic cortex and drop into the yolk (arrowhead). a"'") overview. b) In less than $5 \%$ of the analyzed embryos, massive ubiquitous malformation of the blastoderm is observed. Note the absence of palisadal epithelial morphology and the formation of holes (asterisk). C-c") cellularization defects with a low penetrance are observed in aen ${ }^{2}$ mutants. red: Dlt; green: Nrt; blue: DNA. c) Cross section of a stage 5-2 epithelium. Regionally restricted delay of FC progression (left half). Nrt accumulations at the embryonic cortex. Nuclear density reduced and failure of nuclear elongation (arrowhead). c') Grazing section of the lateral blastoderm. Formation of multinucleate cells (asterisk). c") Cross section of a stage 5-3 epithelium. Palisadal morphology disordered.

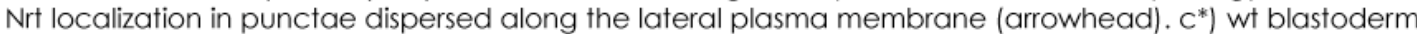
epithelium. Nrt evenly distributed in the plasma membrane. d) Statistical representation of the penetrance of strong cellularization defects in aen-slam double mutant embryos (slam\#: for details of the cross see section 2.2.3), slam single mutant embryos, aen ${ }^{2}$ mutant embryos and wt embryos (as depicted in a); dark: strong phenotypes (local failure of FC progression, multinucleate cells), light no/weak phenotype. $n$ denotes the number of embryos counted.

The analysis of the single mutants gave the following picture: In slam single mutant embryos $5,4 \%$ of the embryos show strong defects in blastoderm cellularization, characterized by a local reduction of the invagination depth of the FC, covering one of the poles and 
extending towards the median of the embryo. A clear majority of $94,6 \%$ shows no such defects.

In homozygous aen2 mutant embryos, the penetrance of cellularization defects is elevated in comparison to slam/defiency mutants and wt. $23 \%$ of the embryos exhibit a partial failure of blastoderm cellularization (figure 17c). Interestingly, a partial mislocalization of Nrt to the apical cytoplasm, similar to the defect reported for maternal-zygotic slam mutants, can be observed in some cases. With a low penetrance of less that $5 \%$ the formation of multinucleate cells is observed (17c'). Also severe disruption of the palisadal morphology of the somatic epithelium is observed with a low penetrance. In these cases, Nrt mislocalization in punctate enrichments in the lateral membranes occurs (figure 17c"). However, blastoderm cellularization was unaffected in $77 \%$ of the embryos.

After the characterization of the single mutants, the phenotype of the slam-aen double mutant was analyzed. It was expected that a biologically relevant interaction between the two factors would result in a strong induction of the cellularization- or PGC migration phenotypes observed in the single mutants.

It was found that the majority of offspring originating from slam heterozygous mothers exhibited a variety of cellularization defects. With almost full penetrance, a filling of the micropyle, a small appendix at the anterior pole of the vitellin membrane surrounding the embryo (figure 2a), with cytoplasm was observed Also nuclei were frequently found to invade the micropyle. Strong cellularization defects which included asynchrony of cellularization where observed in $45 \%$ of the embryos. In these embryos, a local reduction of invagination depth of the FC occurs. Furthermore, the incomplete formation of the FC and the formation of multinucleate cells were found some in regions of the embryos, resulting in a local complete 
block of the cellularization. In most embryos analyzed, the strongest defects were found at the embryonic poles.

These results indicate that the phenotype found in slam single mutants is strongly enhanced in conjunction with the aen ${ }^{2}$ mutation, adopting the characteristics of the phenotype found in the maternal/zygotic slam null mutants (Stein et al., 2002).

\subsubsection{Cellularization kinetics in aen mutants}

The analysis of slam (Lecuit et al., 2002) had shown that the kinetics of FC invagination is altered by RNAi-mediated slam knockdown. The membrane invagination speed was found reduced by half in slam knockdown as compared to wt. As the biochemical and genetic interaction and co-localization studies suggests a functional cooperation of Aen and Slam during cellularization, the speed of membrane insertion was investigated in aen mutants during late cellularization phase. For this purpose, embryos were dechorionated and mounted with halocarbon oil. Recordings were taken with phase contrast microscopy, which allows the visualization of the highly light diffracting FC in cross sections of the developing epithelium. The vitellin membrane, which marks the outer boundary of the embryo, was chosen as a reference structure.

The recordings of the fast phase of FC invagination in aen mutant embryos show that the kinetics of membrane invagination is not significantly altered in aen mutants (figure 18a and b). This finding might be a result of the low penetrance of cellularization defects in aen ${ }^{2}$ mutant embryos. 

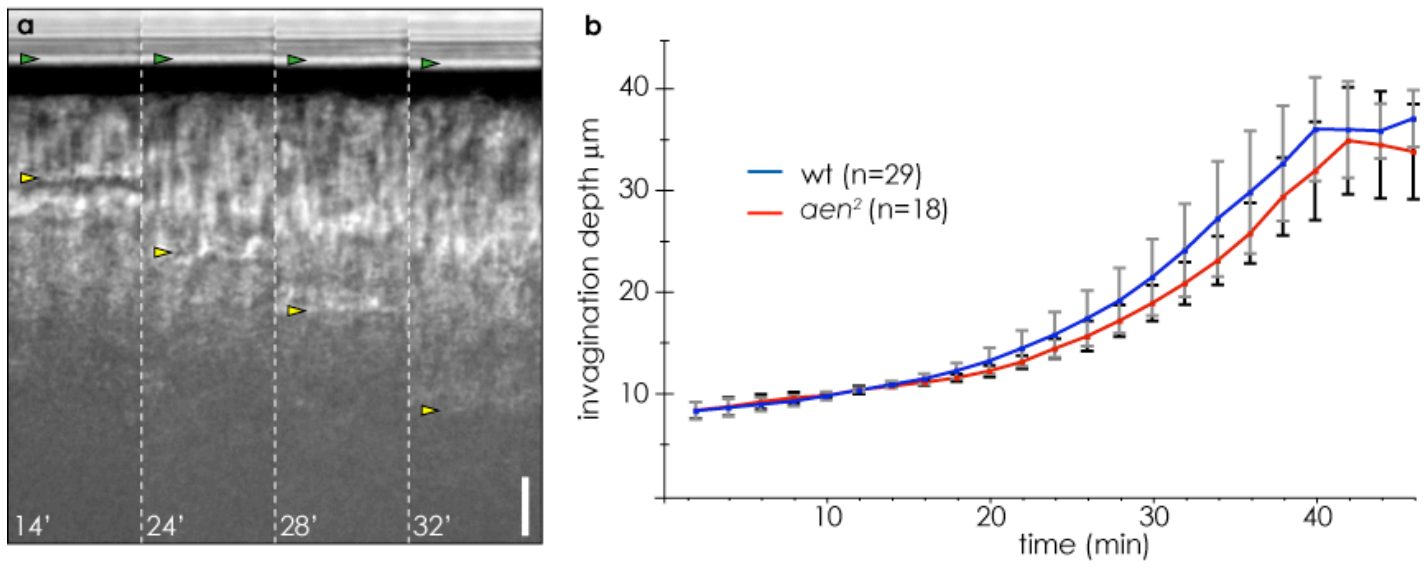

Figure 18: Velocity of membrane invagination in aen ${ }^{2}$ mutants. Phase contrast microscopy image series in wt and aen ${ }^{2}$ mutant embryos. a) cross section of a developing epithelium. Dorsal is up. Scalebar: $5 \mu \mathrm{m}$. The invagination depth of the FC (yellow arrowhead) as a function of time was measured by timelapse imaging. The vitellin membrane (green arrowhead) was used as a mark for the outer border of the embryo. b) Results of the velocity measurements described in a. The comparison of wt with aen ${ }^{2}$ mutants shows that the kinetics of membrane invagination are not significantly affected in the median regions of aen ${ }^{2}$ mutants during the fast phase of blastoderm cellularization. Error bars represent the standard deviation. $\mathrm{n}$ denotes the number of embryos analyzed.

3.7.3 Cuticle phenotypes in single and double mutants of aen and slam

In previous functional studies of factors involved in the establishment of cell polarity and adhesion, the frequent occurrence of cuticle malformation had been observed (Wieschaus, 1984; Müller and Wieschaus, 1996; Knust et al., 1996). The cuticle is the exoskeleton of Drosophila larval and adult stages. The larval cuticle, which is secreted by epidermal cells during late embryogenesis from late stage 15 onwards, fully encloses the embryo and exhibits a characteristic structured form that reflects the identity of the embryonic segments by the pattern of the denticle belts, anterior structures, the head skeleton including the mouthparts, as well as posterior structures with the filzkörper (reviewed in Martinez Arias, 1993). Cuticle preparations have been applied for the analysis of mutant phenotypes in body axis formation and segmentation (e.g. Fröhnhofer and Nüsslein-Volhard 1986). However, cuticle preparations were also used to identify defective cellularization processes. Mutants affecting membrane 
polarity or adhesion (e.g. bazooka (Müller and Wieschaus, 1996, Knust et al., 1996) and crumbs (Tepass et al., 1990; Wodarz et al., 1995; Wodarz et al., 1993; Jürgens et al., 1994); see also section 1.1.3), result in extensive cell death and the disruption of the primary epithelium shortly after cellularization. Therefore, only parts of the surviving tissues secrete cuticle later in development. These cuticles often exhibit partial reductions in the structures described above, contain holes (bazooka (Wieschaus, 1984)) or are completely incoherent and unstructured (crumbs, stardust (Tepass et al., 1990; Müller and Wieschaus, 1996)). These examples show that strong defects in cellularization often are distinctly reflected in the cuticle morphology. To quantify penetrance of the cellularization phenotypes observed in the slam and aen ${ }^{2}$ single mutants as well as in the slam-aen double mutants, the cuticle phenotypes found in these three genotypes were examined and quantified.

For the analysis three categories of cuticle phenotypes were defined. The first category was characterized by the lack of cuticle, which could either reflect extreme cellularization phenotypes resulting in the formation of no epidermal cells whatsoever or the death of formed tissues prior to the secretion of the cuticle. Unfortunately these phenotypes could not be distinguished from unfertilized eggs. Therefore this phenotypic class was excluded from the consecutive statistical analysis. Embryos that exhibited a cuticle lacking all three signs of defined morphology such as denticle belts, headskeleton and filzkörper were grouped in the second category. The third category encompassed embryos that showed either a wildtype cuticle or an incomplete cuticle bearing at least one of the morphologically defined traits.

The analysis of the cuticle preparations yielded the following results (see figure 18). In slam single mutants $90,5 \%$ of unhatched embryos 

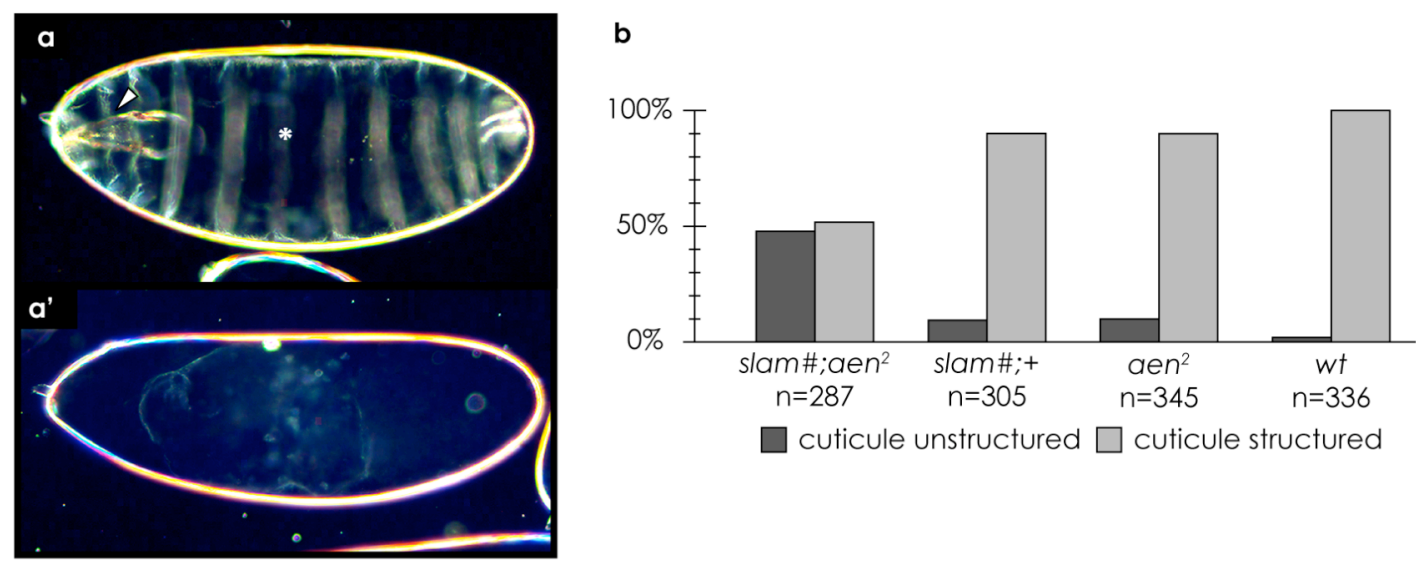

Figure 19: Cuticle phenotypes in slam-aen double mutants. Cuticle preparations of slam-aen double mutant embryos, slam single mutant embryos, aen ${ }^{2}$ mutant embryos and wt embryos. a) Cuticule of a wt embryo exhibiting three morphological characteristics: The headskeleton including the mouthparts (white arrowhead, the denticle belts (asterisk) and the filzkörper at the posterior pole (black arrowhead). a') Severe cuticule phenotype in aen-slam double mutant embryos. The cuticule is fragmented and unstructured bearing neither headskeleton nor denticle belts nor filzkörper. b) Statistical representation of the penetrance of strong cuticule phenotypes (see a'). $n$ denotes the number of embryos counted.

with cuticle exhibited signs of morphological differentiation. In most of the cases all three morphological criteria, headskeleton, filzkörper and denticles were observed. Only $9,5 \%$ of the embryos showed a completely undifferentiated cuticle.

In aen ${ }^{2}$ single mutants a similar observation was made. $90,0 \%$ of the embryos showed a differentiated cuticle while an undifferentiated cuticle was only found in $10,0 \%$ of the cases.

In double mutants the rate of embryos with an undifferentiated cuticle was much higher than in the collections of the two single mutant genotypes: $47,8 \%$ of the embryos belonged to this morphological category. $52,2 \%$ of the embryos showed partially or fully differentiated cuticle morphology. In this group, holes in the cuticle and partial reductions of the head skeleton were frequently observed.

These results show, that aen and slam single and double mutants exhibit cuticle malformations characteristic for cellularization defects. Furthermore, a dramatic increase in phenotypic strength induced by the loss of aen gene activity in slam mutants strongly suggests that a functional interaction exists between the two factors. 


\section{Discussion}

In the present study, the role of the recently identified factor Aeneas (Aen) was characterized as a modulator of primordial germ cell (PGC) migration (section 3.2; Molitor, 2002). Furthermore, Aen is required for the establishment and maintenance of the epithelial integrity during cellularization of Drosophila melanogaster (section 3.7.1). The Aen protein represents a new family of proteins with homologs in a broad range of organisms (section 3.1; figure 6). In aen mutant embryos untimely transmigration of the PGCs occurs with a high frequency during the fast phase of cellularization of the somatic cells of the blastoderm embryo. Aen is expressed both in PGCs as well as in the somatic cells in which it specifically co-localizes with structures of the secretory pathway during cellularization (section 3.4; figures 12 and 13). The investigation of the influence of the aen mutation on the PGCs migration and the cellularization of the underlying epithelium revealed that a functional interplay exists between PGC migration and cytokinesis of the somatic blastoderm. Furthermore, I have identified two factors that interact with the Aen protein, which represent a functional link of Aen activity and the observed cellularization defects. The interaction of Aen with these two factors is shown by two independent biochemical techniques, GST-pulldown assays and co-immunoprecipitation (section 3.5; figure 14). Furthermore, the functional significance of the interaction could be shown for one of the interacting factors by a genetic interaction of the two mutants in vivo (section 3.7; figures 17 and 19). The results obtained in the work presented here indicate that aen loss of function (LOF) affects subcellular protein transport mediated by the Dynactin complex in the somatic blastoderm cells. This transport is involved in 
the polarized insertion of membranes in the plasma membrane (PM) during the cellularization of the somatic epithelium (Lecuit and Wieschaus, 2000; Sisson et al., 2000; Papoulas et al., 2005). aen LOF results in a partial malformation of the somatic blastoderm (section 3.7.1; figure 17) which might reduce its resistance to the transmigration of PGCs. Furthermore, biochemical interaction and functional genetic analysis reveal that Slow as molasses (Slam) represents one of the Aen interacting activities required for cellularization of the somatic epithelium (sections 3.5 and 3.7).

\subsection{Correlation between aen expression, phenotype and localization}

\subsection{1 aen expression}

The expression and localization data presented in this work show that aen expression initiates in the nurse cells of the ovariole after oogenesis stage 8 and that aen expression is restricted to the germline during oogenesis. This finding shows that aen is neither required during early oogenesis nor in the somatic parts of egg follicles (section 3.3.1; figure 9). After transport of the aen mRNAs into the oocyte as a maternal contribution, aen transcripts can be detected ubiquitously distributed until embryonic stage 4 (Molitor, 2002). At stage 5, aen transcripts are degraded in the somatic blastoderm but not the PGCs. Weak ubiquitous zygotic expression is detectable during the following stages in in situ hybridisations and by RT-PCR (Molitor, 2002).

The developmental western blot analysis shows, that Aen protein is first detectable during oogenesis as shown for the transcripts (section 3.3.3; figure 9). In contrast to the transcript localization during early embryogenesis however, Aen protein is not restricted to the PGCs as revealed by whole mount antibody stainings (section 3.4; figures 10 and 11). Instead, Aen can be found ubiquitously during early embryogenesis and also at later stages both in the somatic cells and 
in the PGCs. While the specific stabilisation of the aen transcripts in the PGCs is indicative of a PGC specific function, the ubiquitous localization of the Aen protein suggests a general role in all cells.

\subsection{2 aen phenotype}

The aen maternal and zygotic LOF mutant phenotype is characterized by a premature transmigration of PGCs through the underlying somatic blastoderm during embryonic stages 5 to 9 (section 3.2; Molitor, 2002). This phenotype is clearly caused by the loss of aen activity as it can be rescued by introducing a genomic rescue fragment. Furthermore, weak cellularization defects are observed. In extreme cases the cellularization fails in a region close to the poles (section 3.7.1; figure 17c). In those cases, a mislocalization of Neurotactin (Nrt) (Hortsch et al., 1990) can be observed close to the cell cortex in the apical cytoplasm. Later defects have not been observed in aen LOF mutants. The aen mutation is viable, allowing the keeping of mutant flies as a homozygous stock.

\subsubsection{Subcellular localization of Aen in the somatic blastoderm}

The analysis of the subcellular localization in the somatic blastoderm has shown that Aen, in addition to its cytoplasmic distribution, is localized to small granular structures that could represent vesicles involved in transport (section 3.4; figure 11). To approve this model costaining experiments with markers known to be involved in the vesicle transport required for cellularization were performed.

These experiments revealed that Aen co-localizes partially with the Microtubules (MT) (figure $13 \mathrm{~d}-\mathrm{f}$ ) and with Rabll (figure $12 \mathrm{a}-\mathrm{c}$ ), a marker for vesicles involved vesicle transport and for the recycling endosome (RE) (Ullrich et al., 1996; Sasamura et al., 1997; Pelissier et al., 2003). However, the co-staining of Aen and Rabll is specific for 
small particles localized to the apical cytoplasm and is not observed the RE directly (figure $12 \mathrm{a}-\mathrm{c}$ ). As these vesicles co-express markers of the Golgi apparatus as well (Pelissier et al., 2003), co-localization with Lava lamp (Lva) was investigated, which is required for intracellular transport of Golgi vesicles during blastoderm cellularization (Sisson et al., 2000; Papoulas et al., 2005). However, similar to the situation with Rabl1, the co-localization is restricted to small structures in the lateral and apical cytoplasm and cannot be detected in the larger Golgi particles basal of the nuclei (figure $12 \mathrm{~d}$-f). Taken together, Aen colocalizes with small vesicular structures that are Rabl1 or Lva positive and show at least a partial overlap with the MT network indicating that Aen is positioned on vesicles of the secretory pathway (reviewed in Strickland and Burgess, 2004) presumably downstream of the Golgi apparatus.

\subsubsection{Functional correlation of aen with rab 11 mutants}

Rabl1 controls trafficking through the RE in mammals and Drosophila (Dollar et al., 2002; Ulrich et al., 1996). Injection of a dominant negative protein variant inhibits membrane invagination at the slow and fast phase of cellularization (Pelissier et al., 2003). In Drosophila, hypomorphic mutations of Rabll cause furrow canal (FC) malformation leading to the formation of multinucleate cells (see section 1.1.3; Pelissier et al., 2003). In wildtype, Rabl 1 co-localizes with Nrt in vesicular structures, but not in the RE itself (Pelissier et al., 2003). However, in mutants, which affect vesicle budding from the RE, accumulations of Nrt in this organelle can be found (Pelissier et al., 2003). These results indicate that the transport of Nrt-positive vesicles from the RE is dependent on the function of Rabl 1.

The inhibition of membrane invagination found in Rabll LOF resembles the cellularization defects in strongly affected blastoderm regions found in aen LOF mutants (figure 18). Furthermore, Nrt is found 
ectopically enriched in the apical cytoplasm in aen mutant embryos (figure 18; Pelissier et al., 2003), a phenotype similar to the rabl 1 mutant (Pelissier et al., 2003). Despite these similarities, no indications exist in recent publications that Rabll function influences PGC migration in Drosophila (Pelissier et al., 2003, Riggs et al., 2003).

The co-localization of Aen with Rabl1 and the similarity in aen and rabll LOF phenotypes suggest that Aen function is related to intracellular transport. This hypothesis is further supported by the similarities between Aen and Nrt localization. Nrt mislocalization in aen mutants indicates that Aen function might be involved in transport of vesicles towards the newly forming membrane. However, a direct and exclusive functional interaction of Aen proteins with Rab11 is unlikely due to the differences observed in FC formation and PGC migration.

\subsubsection{Functional correlation of aen with the Iva LOF}

Lva is required for intracellular transport of Golgi vesicles during blastoderm cellularization (Sisson et al., 2000; Papoulas et al., 2005). Its functional inhibition causes phenotypes similar to the ones observed in Rabl1 (Papoulas et al., 2005). Injection of antibodies against Lva or single Lva protein domains causes a reduction in membrane invagination speed and discontinuous FC formation (Sisson et al., 2000). A similar effect can be caused by injection of brefeldinA, which inhibits the transport of Golgi vesicles, and colcemid, which depolymerises MT (Sisson et al., 2000; Lecuit and Wieschaus, 2000). The hypothesis of the functional interconnection between MT based intracellular transport of Golgi vesicles and blastoderm cellularization has been substantiated by the finding that Lva binds the Dynactin complex, which acts as an adjunct of motor protein complexes (Foe et al., 1993; Lecuit and Wieschaus, 2000; Sisson et al., 2000; Papoulas et al., 2005). In good agreement with these findings is the mislocalization of Lva and Nrt caused by depolymerization of MT, which leads to the 
accumulation of Lva- and Nrt- positive structures close to the location of the highest Golgi density, in the basal cytoplasm (Lecuit and Wieschaus, 2000; Sisson et al., 2000). The cellularization phenotypes found in aen mutants exhibit similarities with the effects of anti-Lva, brefeldin or colcemid injection. However, no multinucleate cells have been observed in aen mutants, which is in contrast to the observations made after injection of dominant negative Lva (figure 19; Sisson et al., 2000). Furthermore, Nrt is enriched in the apical cytoplasm in aen mutants, similar to rab 11 mutants and to embryos with depolymerised MT (Pelissier et al., 2003; Lecuit and Wieschaus, 2000). In contrast, Lva LOF probably results in an Nrt accumulation next to the Golgi in the basal cytoplasm (see figure 18; Sisson et al., 2000; Lecuit and Wieschaus, 2000). Similar to Rab11, the studies of Lva function do not indicate its involvement in PGC migration; however, this process might not have been observed due to the experimental setup (Sisson et al., 2000; Papoulas et al., 2005).

Taken together, the co-localization of Aen with MT and with Rabl 1 and Lva in small vesicles in the apical cytoplasm and the similarity in the LOF phenotypes suggest that aen function is related to intracellular transport. The apical enrichment of $\mathrm{Nrt}$ in aen mutants suggests, that Aen does not directly influence the transport from the Golgi to the apical cytoplasm. This finding does not support the hypothesis of a direct interaction and functional relation between the Aen proteins and Lva.

\subsubsection{Subcellular localization of Aen in the PGCs}

The localization of Aen protein in the PGCs differs considerably from the somatic tissues. In PGCs Aen is enriched in large granular structures that are neither Golgi nor RE and show no visible overlap with the MT. So the involvement of Aen proteins in intracellular transport in the PGCs is not probable. Based on their morphology, Aen-positive 
structures observed in these cells could represent polar granules (PGs) (see section 1.1.2; Lehmann and Nüsslein-Volhard, 1986; Hay et al., 1988).

However, characterized factors involved in PG assembly and function, including oskar (osk) and vasa (vas), are deposited specifically at the posterior pole of the oocyte already during oogenesis (see section 1.1.1; Lehmann and Nüsslein-Volhard, 1986; Hay et al., 1988, 1990). During early embryogenesis the mRNAs of these factors as well as the respective proteins are localized to the pole plasm at the posterior pole of the embryo (see section 1.1.2; Lehmann and Nüsslein-Volhard, 1986; Hay et al., 1988, 1990). In contrast, aen transcripts are ubiquitously localized in the oocyte and during embryonic stages 1-4. During stage 5, aen transcripts are restricted to the posterior pole including the PGCs (section 1.3; figure 9; Molitor 2002) but Aen protein is ubiquitously detectable during early embryogenesis (figure 10). Furthermore, there are clear functional differences. Components of the PG are involved several critical developmental processes during early embryogenesis (section 1.1.2; Lehmann and Nüsslein-Volhard, 1986). In mutants of osk, the assembly of the pole plasm is abolished and no PGCs are formed (Lehmann and Nüsslein-Volhard, 1986). In mutants of vas posterior morphogenesis is affected (Hay et al., 1988). In mutants of polar granule component (pgc), the transcriptional repression in the PGCs is abolished resulting in dedifferentiation and loss of PGCs (Martinho et al., 2004; Deshpande et al., 2004). In aen mutants, PGC formation and posterior morphogenesis are not affected (PGC formation: figure 7; posterior morphogenesis: as inferred from cuticle morphology, data not shown). Additionally aen mutants are fertile, which strongly indicates that PGC maintenance is unaffected (section 3.2.1). Conversely, mutants affecting PG factors in which PGCs are still formed show no indication of PGC mismigration 
phenotypes during blastoderm stages (reviewed in Molyneaux and Wylie, 2004).

The lack of overlap between the mutant phenotypes of aen and PG components indicates that the functional role of Aen is unrelated to the functions of PG components. However, aen mutants exhibit phenotypes that affect PGC development. Therefore, a PGC autonomous function of aen might nevertheless exist.

\subsubsection{Tissue specific requirement of Aen}

The premature transmigration of PGCs through the underlying epithelial sheet in aen mutant embryos could be explained by a cell autonomous function of Aen in the PGCs as its transcript is stabilized and the protein is deposited in high amounts in this tissue. Such a PGC autonomous role of Aen could be proven directly by a PGC specific rescue of the aen mutant phenotype using the GAL4-UAS system. However, during early embryogenesis this experimental approach bears several principal difficulties, which cannot be overcome. After their formation, the PGCs are transcriptionally silent, rendering GAL4UAS induced PGC-specific zygotic expression impossible up to stage 910 (section 1.1.2, reviewed in Williamson and Lehmann, 1996; Van Doren et al., 1998). Maternal GAL4-UAS mediated expression would be sufficient to include aen transcripts into the PGCs, similar to the wildtype situation (Molitor, 2002). However no PGC specificity of aen transcripts could be accomplished with this approach. Several maternal transcripts, including nanos (nos), are restricted to the PGCs by active degradation in the somatic tissues (Wang and Lehmann, 1991; Ephrussi et al., 1991; Lehmann and Nüsslein-Volhard, 1991, Bashirullah et al., 1999). This restriction can be induced in other genes by fusion to the nos-3'-UTR coding region (Bashirullah et al., 1999). However, this system is not efficient enough to suppress the translation 
in posterior somatic cells completely and does therefore not allow for a clear distinction of PGC specific effects (Bashirullah et al., 1999).

An experimental alternative would be the transplantation of wt PGCs on aen mutant embryos. In this experiment, PGCs are extracted from fluorescein labelled donor embryos of stage 3-4 and injected into the periembryonic space at the posterior pole of a recipient embryo (Jaglarz and Howard, 1994). This technique has been utilized to investigate PGC migratory behaviour during stage 10 (Jaglarz and Howard, 1994; Jaglarz and Howard, 1995, Kunwar et al., 2003), however several problems make this solution inapplicable for the investigation of the aen phenotype. The retrieval and transplantation is a strong disturbance of the PGC surface morphology. The narrow time window between PGC injection and phenotypic observation would not allow for an appropriate recovery phase. Accordingly, nonspecific effects of this physical agitation, which might influence the experimental result, could not be excluded. These experimental limitations impair the direct investigation of PGC autonomous effects of Aen.

An indirect solution for this problem is offered by the possibility of a specific zygotic rescue of the aen mutant phenotype. If Aen would be required cell autonomously in the PGCs, the expression of Aen in the somatic blastoderm should have no effect on PGC mismigration. A specific somatic expression could be obtained using the Nullo-GAL4 driver line that enables specific expression in somatic cells of the blastoderm embryo during cellularization (Kunwar et al., 2003). Since this soma specific expression of Aen results in a partial rescue of the PGC transmigration phenotype in aen ${ }^{2}$ mutants (section 3.1.4) an exclusive requirement of aen in the PGCs can be ruled out.

Even though this finding does not directly disprove the hypothesis of an additional PGC autonomous function of Aen, it strongly suggests 
that the cause of PGC mismigration is to be found in somatic blastoderm.

Despite the finding that Aen is required in the somatic blastoderm and that it partially co-localizes with components of the secretory pathway, the integration of Aen in a network of interaction partners with characterized functions was necessary for its functional characterization.

\subsection{The interaction between Arpl and Aen}

The first indication towards potential interacting partners of Aen was gained from a genome wide yeast two-hybrid (Y2H) approach. In this high throughput screen, full-length cDNAs of annotated Drosophila genes were utilized to generate an interaction map of the whole Drosophila proteome (Giot et al., 2003). Based on the technical limitations of a high throughput screen, a critical evaluation of the identified candidates on the basis of their expression profiles and subcellular localizations is required. Among the several factors that showed interaction with Aen, the interaction with the highest confidence was found between Aen and Arpl (Fryrberg et al., 1994).

In the light of the previous observations, which had established a possible link between Aen function and intracellular transport, Arp 1 appeared to be a promising candidate for further analysis. Therefore the interaction between AenA and Arpl was tested by means of GSTPulldown and Co-IP experiments. The results of both experimental designs approve the $\mathrm{Y} 2 \mathrm{H}$ interaction of Aen with Arpl (section 3.5.1; figure 14 a and b). 


\subsubsection{Function of Arpl}

Arpl is a major constituent of the Dynactin complex, forming a short filament at the base of the complex (Schafer et al., 1994; Bingham and Schroer, 1999; Imai et al., 2006). Dynactin is involved in multiple transport processes including dynamic functions, like Golgi dynamics and fast axonal transport, and static functions, including the organization of mitotic spindles, chromosome segregation and nuclear positioning (reviewed in Karki and Holzbaur, 1999; Schroer, 2004; Sharp et al., 2000; Whited et al., 2004; Karess, 2005; Kim et al., 2007). In the static functions of Dynactin, its direct interaction with MT via the Glued subunit plays an essential role (Kim et al., 2007). Dynactin has been shown to be present at the attachment site of MT to the PM and to kinetochores during mitosis and has been hypothesized to organize signaling molecules by tethering them to the MT (Geiser et al., 1997; Schroer 2004). Dynactin interacts with the motor protein complexes Dynein and Kinesin2 and is involved in their function (reviewed in Schroer, 2004; Deakon et al., 2003). The Arpl filament has been shown to bind proteins localized at vesicle surfaces, including $\beta$ III Spectrin, a component of Golgi membranes, and COPII, which is involved in the vesicle transport from ER to Golgi (Beck et al., 1994; Holleran et al., 2001; Watson et al., 2005; COPll function reviewed in Sato et al., 2007). Therefore it has been suggested that the Dynactin complex acts as a cargo receptor for motor complexes, mainly Dynein. In good agreement with this finding is the colocalization of Dynactin with Golgi associated vesicles (Habermann et al., 2001). In contrast, recent work by Haghnia et al., (2007) suggests that the complex is nonessential for membrane-recruitment of motor proteins. 


\subsubsection{Phenotypes of Dynactin LOF}

Dynactin LOF phenotypes in Drosophila are observed in a variety of developmental processes, including oogenesis, spermatid growth, larval motility and eye formation (section 1.1.1; Haghnia et al., 2007; Gosh-Roy et al., 2005; Duncan and Warrior, 2002; Fan and Ready, 1997; Reddy et al., 1997; Fan, 2004). The requirement of Dynactin during oogenesis manifests in a failure of oocyte differentiation in Arp 1 mutant ovarioles, similar to the phenotypes observed in bicaudalD (bicD) and egalitarian (egl) mutants (section 1.1.1; Mach and Lehmann, 1997; Haghnia et al., 2007). When Dynactin function is impaired during late oogenesis, the deposition of morphogens during anteroposterior axis formation in the oocyte is affected (Januschke et al., 2002).

Due to the experimental constraints of early embryogenesis in Drosophila, the function of Dynactin during blastoderm cellularization has not been investigated directly yet. However, a recent study shows, that inhibition of the Dynein motor complex during blastoderm cellularization causes effects, closely resembling the phenotypes induced by IVa LOF or MT depolymerization (section 1.1.3; section 4.1.4; Papoulas et al., 2005). Embryos from transheterozygous dynein heavy chain ( $d h c)$ hypomorphic mutant mothers develop until mitosis 14 but then fail to cellularize (Papoulas et al., 2005). The localization of Lva-positive structures in these embryos is, in contrast to wt restricted to the basal side of the nuclei (Papoulas et al., 2005). Furthermore, the injection of specific antibodies against Dhc results in a strong reduction of membrane invagination speed during both the slow and fast phase of cellularization (Papoulas et al., 2005). On the basis of the extensive characterization of the cooperative function of Dynactin and Dynein (reviewed in Karki and Holzbaur, 1999; King et al., 2003), these phenotypes reflect the phenotypes that would be caused by a 
direct inhibition of the Dynactin complex function with a high probability.

\subsubsection{Functional correlation between Dynactin and Aen}

The comparison of aen phenotypes with the phenotypes described for dynactin LOF clearly shows that many processes that require Dynactin activity are unaffected in aen mutants. This applies to the Dynactin function in axis formation. The presence of Aen proteins during late oogenesis might indicate a requirement of Aen function in Dynactindependent morphogen deposition (figure 9; Januschke et al., 2002). However, the axis formation is not affected in aen mutants, as shown by the unipolar positioning of PGCs and the absence of characteristic morphological defects later in development. Phenotypes in postembryonic stages attributed to dynactin LOF, like larval posterior paralysis or rough eye phenotypes in adults (Haghnia et al., 2007; Karki and Holzbaur, 1999), have not been observed in aen mutants. These observations show, that Aen is no constitutive functional adjunct of the Dynactin complex. Conversely, no PGC migration defects attributed to dynactin LOF have been described in literature (reviewed in Karki and Holzbaur, 1999; Papoulas et al., 2005)

During cellularization, however, the almost complete co-localization of Aen and the Dynactin associated motor protein Dhc suggests a functional interaction between these factors (figure 16). The similarity between phenotypes induced by $d h c$ LOF and aen LOF, both characterized by a partial failure of blastoderm cellularization (Papoulas et al., 2005), supports this hypothesis along with the biochemical data (see also section 4.1.3; figure 14). These data, in connection with the co-localization of Aen proteins with components of the secretory pathway (figure 12), strongly suggests that Aen is involved in intracellular transport during cellularization. The ectopic accumulations of $\mathrm{Nrt}$ in the apical cytoplasm of affected regions of 
aen mutants indicate that Nrt is still transported in apical direction suggesting that the membrane transport is affected downstream of the Golgi (Sisson et al., 2000; Lecuit and Wieschaus, 2000; Papoulas et al., 2005). The co-localization of Aen and Rabll shows that Aenpositive vesicles reach the RE and are normally processed as indicated by the absence of ectopic Nrt enrichments directly in the RE (section 1.1.3; figure 18; section 4.1.3; Pelissier et al., 2003). Thus the effect of the aen mutation most probably affects a process that functionally is arranged in between the budding of secretory vesicles from the RE and their fusion to the PM.

In order to further narrow down the functional role of Aen in secretion, further candidates for a direct interaction with the Aen proteins needed to be identified.

\subsection{The interaction between Slam and Aen}

The identification of Slam as a potential interaction partner of Aen was due to its described involvement in cellularization and PGC migration (Lecuit et al., 2002; Stein et al., 2002). Similar to Arpl, the binding of Slam to AenA was tested by means of GST-Pulldown and Co-IP. It was found, that the two proteins interact and exhibit a partial colocalization, which covers the FC and the basolateral membrane.

The Slam protein has been shown to be involved in the formation of the FC and the basal adherence junction (BAJ) (Lecuit et al., 2002). It is required to recruit other components of the FC and the contractile ring to the forming furrow (section 1.1.3, Lecuit et al., 2002). Slam has been suggested to mediate the deposition of yet uncharacterized factors that affect PGC migration at stage 10 after their transmigration through the primordial midgut (PMG) (section 1.1.4; Stein et al., 2002). The phenotypes of amorphic Slam LOF alleles include a malformation of the FC, which is not cleared of villous projections prior to 
invagination (Lecuit et al., 2002). Invagination of the FC is delayed and progresses at about half the velocity of wt during the fast phase of cellularization (Lecuit et al., 2002). In good agreement with its localization to the FC and to the basolateral membrane, is the finding that slam LOF affects the formation of the BAJ and the contractile ring (Lecuit et al., 2002). The localization of several factors including Armadillo, the PDZ-protein D-PATJ, the motor protein Myosin-ll is dependent on Slam (Lecuit et al., 2002). Also the transport of Nrt has been found to be affected in slam LOF, leading to its ectopic localization in the apical cytoplasm (Lecuit et al., 2002). Therefore, it has been hypothesized that the integration of vesicles to the exocytic domains of the PM is affected in Slam mutants (Lecuit et al., 2002). By comparison with amorphic alleles, the phenotypes of hypomorphic alleles occur in later developmental stages. In these embryos, the migration of PGCs is affected (Stein et al., 2002). The defects observed are characterized by an inhibition of the transit of PGCs from the PMG to the mesoderm (section 1.1.4; Stein et al., 2002).

The phenotypes described for maternal-zygotic, hypomorphic Slam LOF and amorphic slam LOF mutants show cellularization defects that are characterized by a delay of membrane invagination resulting in a failure of blastoderm cellularization (Lecuit et al., 2002). Furthermore, $\mathrm{Nrt}$ is localized in the apical cytoplasm instead of the PM. Although no delay in cellularization was detectable in aen mutants, Nrt is mislocalized similar to slam mutant embryos.

However, the PGC migration defects observed in zygotic, hypomorphic slam mutant embryos (Stein et al., 2002) do not resemble those observed in aen mutant embryos. No premature PGC transmigration during stages 5 to 8 is observed in hypomorpic slam mutants. Conversely, no abnormal migration of PGCs after stage 9 
occurs in aen mutant embryos. Therefore the processes causing PGC mismigration in slam mutants are most probably independent of aen.

To show whether Aen and Slam interact on a functional level during blastoderm cellularization, genetic interaction experiments were done utilizing a hypomorphic mutation (Stein et al., 2002) in presence of a maternal contribution. The results of these experiments show that phenotypes observed in slam-aen double mutants are strongly increased in frequency and intensity as compared to slam and aen single mutants (sections 3.7.1 and 3.7.3; figures 17 and 19). This phenotypic increase is not due to the addition of independent slam related and aen related defects, but clearly exceeds the added effects of the single mutants.

The increase phenotypic strength could be interpreted as a qualitative shift from a hypomorphic to an amorphic phenotype, which can no longer be rescued by the maternal contribution (Lecuit et al., 2002; Stein et al., 2002). This shift might be explained by the decrease of Slam protein concentration at the place, where it is normally localized and functionally required. This would mean that the concentration in hypomorphic mutants is high enough to maintain Slam function. If the concentration of Slam is further decreased by removing the maternal contribution or as a consequence of aen LOF, the concentration sinks below a critical threshold and cellularization defects occur. Such a model has been offered by Stein et al (2002) to explain the phenotypes induced by removal of the maternal contribution in the hypomorphic mutants. Alternatively, the phenotypic shift is not caused in dependence of Slam concentration, but represents a decrease of functional efficiency, which could be due to the missing of Aen as a functional adjunct. 
4.3.1 Functional models for Aen Slam interaction

1) Slam transport: Aen acts as a specific cargo receptor, which recruits Slam to the Dynein-Dynactin transport complex. Upon binding Aen, Slam is transported to the sites of the prospective FC.

In addition to the biochemical and genetic interaction studies, this hypothesis is supported by the close co-localization of Aen with the transport complexes and secretory vesicles on one and its association with Slam adjacent of the FC and the basolateral membrane on the other side. The strong enhancement of the zygotic slam phenotypes found in the double mutants could be explained with this model. In the slam single mutant, low levels of maternal supplied Slam are sufficient to maintain cellularization. If the efficiency of transport of Slam to the prospective FC is decreased because of the lack of Aen as a specific cargo adaptor, the Slam concentration at the FC is reduced beyond a critical threshold and cellularization fails.

2) Slam mediated membrane recruitment to the FC: In addition to the recruitment of cargo to the motor complexes the intersection of Aen with Slam could enable specific membrane targeting.

Slam might mediate specific recruitment of Aen-decorated cargo to the basolateral membrane and enable subsequent membrane fusion. This targeting of transport might be involved in the establishment of adhesive structures and membrane polarity. This hypothesis would be in agreement with the subcellular localization of the factors involved and might also explain the phenotypes observed in slam-aen double mutants. Furthermore, this essential function of the Slam-Aen interaction would explain, why Aen is not required in all Dynactin complex functions. However, the interaction of Aen and Slam cannot 
be essential for the proper localization, as the aen phenotype is not identical to the slam maternal zygotic phenotype. Therefore, Aen seems be required for membrane fusion but not essential for the targeting of membranes.

3) Constitutive Aen Slam interaction at the FC: Aen might cooperate with Slam in the establishment of a membranetargeting complex that promotes polarized membrane insertion at the basolateral membrane.

Aen might provide a physical link between the BAJ and the MT cytoskeleton by connecting the Dynactin complex to Slam. This function might support BAJ integrity and also facilitate the polarized transport of secretory and transcytotic vesicles. This hypothesis is in partial agreement with the phenotypes observed in aen-slam double mutants. However a direct involvement of Dynactin in adherence junction assembly and maintenance during embryogenesis has not been shown yet. Furthermore, this hypothesis would not explain the large pools of cytoplasmic and vesicle associated Aen proteins (figure 10; figure 11; figure 12).

These functional models however do not include an explanation for the PGC mismigration observed either in aen or in slam mutants. The most obvious explanation for the premature transmigration of PGCs through the PMG would be a weakening of the epithelial integrity.

The analysis of aen and slam mutants reveals some phenotypes that are in good agreement with a defective epithelial integrity. The phenotypes observed in amorphic slam mutants include the malformation of adhesive structures and affect membrane polarity (Lecuit et al., 2002). Moreover, the adhesion factor Nrt is mislocalized (Hortsch et al., 1990). Similarly, in a subset of aen mutant embryos mislocalization of Nrt can be observed as well (figure 7). In addition, 
the analysis of the cuticle structures of aen-slam double mutant larvae shows a dramatic loss of cuticle structure (figure 18). This phenotype is highly reminiscent of phenotypes observed in mutants of bazooka (baz), a factor required for the establishment of epithelial polarity (section 1.1.4; Müller and Wieschaus, 1996; Knust et al., 1996). This indicates that the epithelial integrity is strongly affected in aen-slam double mutant embryos suggesting that both factors are cooperatively involved in the establishment of the epithelial barrier function. Therefore, it would be expected that the integrity of the epithelium is also affected in the hypomorphic slam mutant as well as in the aen mutant. The mislocalization of Nrt in both mutants and the untimely PGC transmigration in aen mutants support this hypothesis. So, based on the idea that untimely PGC transmigration is caused by a reduction in epithelial integrity, PGC migration could be used as an independent assay to screen for defective epithelial integrity. However, the lack of premature transmigration in slam single mutants shows that untimely PGC transmigration might only occur if the integrity is dramatically weakened - as in the double mutant - or alternatively, that the PGC transmigration in aen mutants is not monocausally induced by the reduction of epithelial integrity but rather reveals an additional function of aen. Therefore Aen might be involved in the transport of signaling factors required for the modulation of PGC migration.

1.5.2. Signaling pathways potentially involved in premature PGC migration in aen mutants

In the wt situation PGC migration occurs after embryonic stage 9 When the close adherence of the PGCs is weakened (Jaglarz and Howard, 1994). In addition, epithelial transmigration is controlled by the cells of the PMG, as a transformation of these cells into hindgut cells in huckebein mutants blocks of transmigration completely 
(Jaglarz and Howard, 1994; Reuter 1994; Moore et al., 1998). The importance of the PMG cells is further shown by transplantation and in vitro experiments (Jaglarz and Howard, 1994; Jaglarz and Howard, 1995). When PGCs are explanted from stage 5 embryos and kept in primary cell culture, they start to migrate. When instead they are transplanted into embryos, they do not migrate through the PMG epithelium until stage 9 begins (Jaglarz and Howard, 1995). By isochronic and heterochronic transplantation experiments, where the age of donor and recipient embryo were either matched or leading or lagging by up to $3 \mathrm{~h}$, it was shown that the time of PGC transmigration is not determined by the age of the PGCs, but rather by the stage of the midgut (Jaglarz and Howard, 1994). Based on these results transmigration of PGCs is controlled by signaling from PMG cells.

\subsubsection{JAK/STAT signaling in PGC migration}

The only signaling cascade, which has been shown to influence PGC migration during stage 5 , is the Janus kinase/signal transducer and activator of transcription (JAK/STAT) signaling pathway (reviewed in Hou et al., 2002, Brown et al., 2006). In the PGCs, the JAK/STAT system functions downstream of the receptor tyrosine kinase (RTK) Torso (Tor) (Li et al., 2003; reviewed in Duffy and Perrimon, 1994) and has been shown to influence both early PGC division and migration.

Components of the JAK/STAT signaling pathway, including hopscotch, encoding the Drosophila Jak, and Stat92E (Hou et al., 1996; Li et al., 2003) exhibit a similar localization than Aen (Li et al., 2003). Transcripts are maternally deposited and ubiquitous during early embryogenesis followed by a stabilization of the mRNA in the PGCs after stage 3 (Li et al., 2003). The phenotypes observed in LOF and gain of function (GOF) variants of components of the JAK/STAT pathway (Li et al., 2003; Brown et al., 2006) suggest that the signaling pathway is involved in several aspects of PGC development. In Stat92E mutants, the formation of 
filopodia during active migration is compromised (Jaglarz and Howard, 1995; Brown et al., 2006) and consecutively, PGC migration at stage 10-11 is delayed or inhibited (Li et al., 2003; Brown et al., 2006).

Striking phenotypes are also observed in the GOF situation. The analysis of a hopscotch (hop) GOF allele, which leads to overactivation of the pathway (Binari and Perrimon, 1994) showed an overproliferation of the PGCs. Furthermore, the PGCs show an increased migratory behavior resulting in their translocation from the posterior pole. However, in contrast to the aen phenotype, the PGCs do not only transmigrate but tanslocate on the embryonic surface, leading to dispersed PGC clusters that extend into anterior direction. However, later at stage 8-9 the PGCs show premature migration through the PMG epithelium (Li et al., 2003) resulting in PGCs in ectopic locations during later stages. Furthermore, the ectopically localized PGCs appear to be stabilized in hop GOF (Li et al., 2003), while in wt they are eliminated (Coffman et al., 2002).

Despite the occurrence of premature PGC transmigration phenotypes in both aen mutant and hop GOF, a direct functional interaction of aen with the JAK/STAT pathway appears unlikely. The phenotypes caused by STAT overactivation demonstrate a general increase in the migratory behavior of PGCs resulting in a migration along the embryonic surface, while only a subset of PGCs transmigrates the blastoderm epithelium (Li et al., 2003). In aen mutants, migration along the embryonic surface has not been observed. This observation is counterindicative of a general increase in migratory potential of PGCs in aen mutants. Moreover, a genomewide RNAi screen for so far uncharacterized components of the JAK/STAT pathway (Müller et al., 2005) has given no indication of a direct implication of Aen in JAK/STAT signaling. However a potential mutual influence of Aen and JAK/STAT signaling remains an interesting option. 


\subsubsection{Tre 1/Wun signaling in PGC migration}

The G-protein coupled receptor Trapped in endoderm-1 (Tre1) in a signaling factor involved in the initiation of PGC migration through the PMG (Kunwar et al., 2003). In tre 1 mutant embryos the PGCs remain trapped in the PMG pocket at stage 10 (Kunwar et al., 2003). The inability to migrate through the PMG does not reflect a general effect on PGC migration as escaping PGCs retain the ability to migrate towards the somatic gonadal precursor cells (SGPS) and become incorporated into the gonads (Kunwar et al., 2003). Trel functions cell autonomously in the PGCs as shown by transplantation and GAL4UAS-mediated rescue experiments. tre 1 mRNA is like aen maternally contributed and stabilized in the PGCs. However, no antibody is available to show if the Trel protein is PGC specific (Kunwar et al., 2003).

These data indicate that an attractive signal from the PMG is required to allow PGC transmigration at stage 9. Based on the trel mutant phenotype a potential explanation for the PGC migration observed in aen mutants could be a premature signal from the PMG cells to the PGCs at late stage 5 instead of stage 9 in wildtype. The ectopic secretion of an attractive signal would be contradictory to the reduced secretion efficiency indicated by Nrt mislocalization in aen single- and slam-aen double mutants.

Another possibility would be that Aen is acting as a negative regulator of trel signaling in the PGCs. However, based on the partial rescue of the transmigration phenotype in aen mutants by specific expression in the somatic cells a PGC autonomous effect can be ruled out.

Wunen (Wun) and Wunen2 (Wun2) are phospholipid phosphatases (section 1.1.4; Zhang et al., 1996; Starz-Gaiano et al., 2001; Renault et al., 2004) and have been hypothesized to act in concert with Trel in 
influencing PGC migration during stages 9 to 11 . Somatic tissues expressing Wun and Wun2 have been suggested to repel PGCs by depleting their environment from an otherwise ubiquitous attractive signal (Renault et al., 2004). However, neither do wun and wun2 mutants exhibit premature PGC mismigration (Zhang et al., 1996; StarzGaiano et al., 2001) nor does PGC mismigration occur in aen mutants at stages 9 to 11 . This clearly suggests that aen phenotypes are independent of Wun signaling.

\subsection{Outlook}

In Drosophila, several factors have been identified that participate in the regulatory and physical interaction between migratory cells and tissue. However, the understanding of the functional network required for initiation and permission of tissue invasion is still incomplete (reviewed in Kunwar et al., 2006). In this work, it was shown that Aen participates in the transport of de novo synthesized factors to the PM during the cellularization of the somatic blastoderm. aen mutants exhibit premature PGC transmigration phenotypes. These phenotypes might reflect the mislocalization of factors, which otherwise prevent PGC transmigration until embryonic stage 9.

Therefore Aen might assist in the identification of novel signaling factors involved in the regulation of PGC transmigration. For this purpose, further interaction partners of Aen could be identified by pulldown from embryonic extracts and subsequent massspectrometrical analysis. Additionally the homozygous aen ${ }^{2}$ mutation could be used as a sensitized genetic background for EMS mutagenesis to identify mutations inducing infertility as a consequence of complete premature PGC transmigration.

These experiments might contribute to the identification of molecular programs that control cell migration and tissue invasion. 


\section{Literature}

Altschul, S.F., W. Gish, W. Miller, E.W. Myers, and D.J. Lipman. 1990. Basic local alignment search tool. J Mol Biol. 215:403-10.

Anderson, K. V., and Nüsslein-Volhard, C. (1984). Information for the dorsal-ventral pattern of the Drosophila embryo is stored as maternal mRNA, Nature. 31 1:223-7.

Ashburner, M. 1989. Drosophila, A Laboratory Handbook. Cold Spring Harbor Laboratory Press.

Bachmann, A., M. Schneider, E. Theilenberg, F. Grawe, and E. Knust. 2001. Drosophila Stardust is a partner of Crumbs in the control of epithelial cell polarity. Nature. 414:63843.

Bachmann, A., M. Timmer, J. Sierralta, G. Pietrini, E.D. Gundelfinger, E. Knust, and U. Thomas. 2004. Cell type-specific recruitment of Drosophila Lin-7 to distinct MAGUKbased protein complexes defines novel roles for Sdt and Dlg-S97. J Cell Sci. 117:1899909.

Bashirullah, A., S.R. Halsell, R.L. Cooperstock, M. Kloc, A. Karaiskakis, W.W. Fisher, W. Fu, J.K. Hamilton, L.D. Etkin, and H.D. Lipshitz. 1999. Joint action of two RNA degradation pathways controls the timing of maternal transcript elimination at the midblastula transition in Drosophila melanogaster. Embo J. 18:2610-20.

Beck, K.A., J.A. Buchanan, V. Malhotra, and W.J. Nelson. 1994. Golgi spectrin: identification of an erythroid beta-spectrin homolog associated with the Golgi complex. J Cell Biol. 127:707-23.

Bhat, M.A., S. Izaddoost, Y. Lu, K.O. Cho, K.W. Choi, and H.J. Bellen. 1999. Discs Lost, a novel multi-PDZ domain protein, establishes and maintains epithelial polarity. Cell. 96:833-45.

Bier, E., H. Vaessin, S. Shepherd, K. Lee, K. McCall, S. Barbel, L. Ackerman, R. Carretto, T. Uemura, E. Grell, and et al., 1989. Searching for pattern and mutation in the Drosophila genome with a P-lacZ vector. Genes Dev. 3:1273-87.

Bilder, D., and N. Perrimon. 2000. Localization of apical epithelial determinants by the basolateral PDZ protein Scribble. Nature. 403:676-80.

Bilder, D., M. Schober, and N. Perrimon. 2003. Integrated activity of PDZ protein complexes regulates epithelial polarity. Nat Cell Biol. 5:53-8.

Binari, R., and N. Perrimon. 1994. Stripe-specific regulation of pair-rule genes by Hopscotch, a putative Jak family tyrosine kinase in Drosophila. Genes Dev. 8:300-12.

Bingham, J.B., and T.A. Schroer. 1999. Self-regulated polymerization of the actinrelated protein Arp1. Curr Biol. 9:223-6.

Bodmer, R. 1993. The gene tinman is required for specification of the heart and visceral muscles in Drosophila. Development. 118:719-29. 
Bonini, N.M., W.M. Leiserson, and S. Benzer. 1993. The eyes absent gene: genetic control of cell survival and differentiation in the developing Drosophila eye. Cell. 72:379-95.

Boyle, M., N. Bonini, and S. DiNardo. 1997. Expression and function of clift in the development of somatic gonadal precursors within the Drosophila mesoderm. Development. 124:971-82.

Boyle, M., and S. DiNardo. 1995. Specification, migration and assembly of the somatic cells of the Drosophila gonad. Development. 121:1815-25.

Brandt, A., F. Papagiannouli, N. Wagner, M. Wilsch-Brauninger, M. Braun, E.E. Furlong, S. Loserth, C. Wenzl, F. Pilot, N. Vogt, T. Lecuit, G. Krohne, and J. Grosshans. 2006. Developmental control of nuclear size and shape by Kugelkern and Kurzkern. Curr Biol. 16:543-52.

Brand, A.H., and N. Perrimon. 1993. Targeted gene expression as a means of altering cell fates and generating dominant phenotypes. Development. 118:401-15.

Broihier, H.T., L.A. Moore, M. Van Doren, S. Newman, and R. Lehmann. 1998. zfh-1 is required for germ cell migration and gonadal mesoderm development in Drosophila. Development. 125:655-66.

Brönner, G., and H. Jäckle. 1996. Regulation and function of the terminal gap gene huckebein in the Drosophila blastoderm. Int J Dev Biol. 40:157-65.

Brown, S., N. Hu, and J.C. Hombria. 2003. Novel level of signaling control in the JAK/STAT pathway revealed by in situ visualisation of protein-protein interaction during Drosophila development. Development. 130:3077-84.

Brown, S., M.P. Zeidler, and J.E. Hombria. 2006. JAK/STAT signaling in Drosophila controls cell motility during germ cell migration. Dev Dyn. 235:958-66.

Burgess, R.W., D.L. Deitcher, and T.L. Schwarz. 1997. The synaptic protein syntaxin1 is required for cellularization of Drosophila embryos. J Cell Biol. 138:861-75.

Burz, D.S., R. Rivera-Pomar, H. Jäckle, and S.D. Hanes. 1998. Cooperative DNAbinding by Bicoid provides a mechanism for threshold-dependent gene activation in the Drosophila embryo. Embo J. 17:5998-6009.

Caceres, L., and L.A. Nilson. 2005. Production of gurken in the nurse cells is sufficient for axis determination in the Drosophila oocyte. Development. 132:2345-53.

Callaini, G., M.G. Riparbelli, and R. Dallai. 1995. Pole cell migration through the gut wall of the Drosophila embryo: analysis of cell interactions. Dev Biol. 170:365-75.

Campos-Ortega, J.A., and V. Hartenstein. 1985. The embryonic development of Drosophila melanogaster. Springer Verlag, Berlin.

Carpenter, A.T. 1994. Egalitarian and the choice of cell fates in Drosophila melanogaster oogenesis. Ciba Found Symp. 182:223-46; discussion 246-54.

Celniker, S.E., D.J. Keelan, and E.B. Lewis. 1989. The molecular genetics of the bithorax complex of Drosophila: characterization of the products of the Abdominal-B domain. Genes Dev. 3:1424-36.

Cherbas, P., L. Cherbas, and C.M. Williams. 1977. Induction of acetylcholinesterase 
activity by beta-ecdysone in a Drosophila cell line. Science. 197:275-7.

Clark, A., C. Meignin, and I. Davis. 2007. A Dynein-dependent shortcut rapidly delivers axis determination transcripts into the Drosophila oocyte. Development. 134:1955-65.

Clarkson, M., and R. Saint. 1999. A His2AvDGFP fusion gene complements a lethal His2AvD mutant allele and provides an in vivo marker for Drosophila chromosome behavior. DNA Cell Biol. 18:457-62.

Coffman, C.R., R.C. Strohm, F.D. Oakley, Y. Yamada, D. Przychodzin, and R.E. Boswell. 2002. Identification of $X$-linked genes required for migration and programmed cell death of Drosophila melanogaster germ cells. Genetics. 162:273-84.

Cooley, L., E. Verheyen, and K. Ayers. 1992. chickadee encodes a profilin required for intercellular cytoplasm transport during Drosophila oogenesis. Cell. 69:173-84.

Cooperstock, R.L., and H.D. Lipshitz. 2001. RNA localization and translational regulation during axis specification in the Drosophila oocyte. Int Rev Cytol. 203:541-66.

Cumberledge, S., J. Szabad, and S. Sakonju. 1992. Gonad formation and development requires the abd-A domain of the bithorax complex in Drosophila melanogaster. Development. 115:395-402.

de Cuevas, M., and A.C. Spradling. 1998. Morphogenesis of the Drosophila fusome and its implications for oocyte specification. Development. 125:2781-9.

Deacon, S.W., A.S. Serpinskaya, P.S. Vaughan, M. Lopez Fanarraga, I. Vernos, K.T. Vaughan, and V.I. Gelfand. 2003. Dynactin is required for bidirectional organelle transport. J Cell Biol. 160:297-301.

Deshpande, G., G. Calhoun, and P. Schedl. 2004. Overlapping mechanisms function to establish transcriptional quiescence in the embryonic Drosophila germline. Development. 131:1247-57.

Deshpande, G., L. Swanhart, P. Chiang, and P. Schedl. 2001. Hedgehog signaling in germ cell migration. Cell. 106:759-69.

Doitsidou, M., M. Reichman-Fried, J. Stebler, M. Koprunner, J. Dorries, D. Meyer, C.V. Esguerra, T. Leung, and E. Raz. 2002. Guidance of primordial germ cell migration by the chemokine SDF-1. Cell. 111:647-59.

Dollar, G., E. Struckhoff, J. Michaud, and R.S. Cohen. 2002. Rabl 1 polarization of the Drosophila oocyte: a novel link between membrane trafficking, microtubule organization, and oskar mRNA localization and translation. Development. 129:517-26.

Duffy, J.B., and N. Perrimon. 1994. The torso pathway in Drosophila: lessons on receptor tyrosine kinase signaling and pattern formation. Dev Biol. 166:380-95.

Duncan, I. 1986. Control of bithorax complex functions by the segmentation gene fushi tarazu of D. melanogaster. Cell. 47:297-309.

Duncan, J.E., and R. Warrior. 2002. The cytoplasmic dynein and kinesin motors have interdependent roles in patterning the Drosophila oocyte. Curr Biol. 12:1982-91. 
Eckley, D.M., S.R. Gill, K.A. Melkonian, J.B. Bingham, H.V. Goodson, J.E. Heuser, and T.A. Schroer. 1999. Analysis of dynactin subcomplexes reveals a novel actin-related protein associated with the arpl minifilament pointed end. $J$ Cell Biol. 147:307-20.

Eckley, D.M., and T.A. Schroer. 2003. Interactions between the evolutionarily conserved, actin-related protein, Arp 1 1, actin, and Arp1. Mol Biol Cell. 14:2645-54.

Ephrussi, A., L.K. Dickinson, and R. Lehmann. 1991. Oskar organizes the germ plasm and directs localization of the posterior determinant nanos. Cell. 66:37-50.

Ephrussi, A., and R. Lehmann. 1992. Induction of germ cell formation by oskar. Nature. 358:387-92.

Esposito, D., and D.K. Chatterjee. 2006. Enhancement of soluble protein expression through the use of fusion tags. Curr Opin Biotechnol. 17:353-8.

Fan, S.S. 2004. Dynactin affects extension and assembly of adherens junctions in Drosophila photoreceptor development. J Biomed Sci. 11 :362-9.

Fan, S.S., and D.F. Ready. 1997. Glued participates in distinct microtubule-based activities in Drosophila eye development. Development. 124:1497-507.

FlyBase. 1999. The FlyBase database of the Drosophila genome projects and community literature. Nucleic Acids Res. 27:85-88. http://flybase.bio.indiana.edu/

Foe, V.E., G.M. Odell, and B.A. Edgar. 1993. Mitosis and Morphogenesis in the Drosophila Embryo. The Development of Drosophila melanogaster (Cold Spring Harbor Laboratory Press). 1:149-300.

Friedl, P., and K. Wolf. 2003. Tumour-cell invasion and migration: diversity and escape mechanisms. Nat Rev Cancer. 3:362-74.

Fröhnhofer, H.G., R. Lehmann, and C. Nüsslein-Volhard. 1986. Manipulating the anteroposterior pattern of the Drosophila embryo. J Embryol Exp Morphol. 97 Suppl:16979.

Fullilove, S.L., and A.G. Jacobson. 1971. Nuclear elongation and cytokinesis in Drosophila montana. Dev Biol. 26:560-77.

Fyrberg, C., L. Ryan, M. Kenton, and E. Fyrberg. 1994. Genes encoding actin-related proteins of Drosophila melanogaster. J Mol Biol. 241:498-503.

Geiser, J.R., E.J. Schott, T.J. Kingsbury, N.B. Cole, L.J. Totis, G. Bhattacharyya, L. He, and M.A. Hoyt. 1997. Saccharomyces cerevisiae genes required in the absence of the CIN8-encoded spindle motor act in functionally diverse mitotic pathways. Mol Biol Cell. 8:1035-50.

Ghosh-Roy, A., B.S. Desai, and K. Ray. 2005. Dynein light chain 1 regulates dynaminmediated F-actin assembly during sperm individualization in Drosophila. Mol Biol Cell. 16:3107-16.

Giansanti, M.G., S. Bonaccorsi, B. Williams, E.V. Williams, C. Santolamazza, M.L. Goldberg, and M. Gatti. 1998. Cooperative interactions between the central spindle and the contractile ring during Drosophila cytokinesis. Genes Dev. 12:396-410.

Giot, L., J.S. Bader, C. Brouwer, A. Chaudhuri, B. Kuang, Y. Li, Y.L. Hao, C.E. Ooi, B. Godwin, E. Vitols, G. Vijayadamodar, P. Pochart, H. Machineni, M. Welsh, Y. Kong, B. 
Zerhusen, R. Malcolm, Z. Varrone, A. Collis, M. Minto, S. Burgess, L. McDaniel, E. Stimpson, F. Spriggs, J. Williams, K. Neurath, N. loime, M. Agee, E. Voss, K. Furtak, R. Renzulli, N. Aanensen, S. Carrolla, E. Bickelhaupt, Y. Lazovatsky, A. DaSilva, J. Zhong, C.A. Stanyon, R.L. Finley, Jr., K.P. White, M. Braverman, T. Jarvie, S. Gold, M. Leach, J. Knight, R.A. Shimkets, M.P. McKenna, J. Chant, and J.M. Rothberg. 2003. A protein interaction map of Drosophila melanogaster. Science. 302:1727-36.

Gisselbrecht, S., J.B. Skeath, C.Q. Doe, and A.M. Michelson. 1996. heartless encodes a fibroblast growth factor receptor (DFR1/DFGF-R2) involved in the directional migration of early mesodermal cells in the Drosophila embryo. Genes Dev. 10:3003-17.

Gonzalez-Reyes, A., H. Elliott, and D. St Johnston. 1995. Polarization of both major body axes in Drosophila by gurken-torpedo signaling. Nature. 375:654-8.

Gonzalez-Reyes, A., H. Elliott, and D. St Johnston. 1997. Oocyte determination and the origin of polarity in Drosophila: the role of the spindle genes. Development. 124:4927-37.

Grieder, N.C., M. de Cuevas, and A.C. Spradling. 2000. The fusome organizes the microtubule network during oocyte differentiation in Drosophila. Development. 127:4253-64.

Grosshans, J., C. Wenzl, H.M. Herz, S. Bartoszewski, F. Schnorrer, N. Vogt, H. Schwarz, and H.A. Müller. 2005. RhoGEF2 and the formin Dia control the formation of the furrow canal by directed actin assembly during Drosophila cellularisation. Development. 132:1009-20.

Habermann, A., T.A. Schroer, G. Griffiths, and J.K. Burkhardt. 2001. Immunolocalization of cytoplasmic dynein and dynactin subunits in cultured macrophages: enrichment on early endocytic organelles. J Cell Sci. 1 14:229-240.

Haghnia, M., V. Cavalli, S.B. Shah, K. Schimmelpfeng, R. Brusch, G. Yang, C. Herrera, A. Pilling, and L.S. Goldstein. 2007. Dynactin Is Required for Coordinated Bidirectional Motility, but Not for Dynein Membrane Attachment. Mol Biol Cell.

Hay, B., L.Y. Jan, and Y.N. Jan. 1988. A protein component of Drosophila polar granules is encoded by vasa and has extensive sequence similarity to ATPdependent helicases. Cell. 55:577-87.

Hay, B., L.Y. Jan, and Y.N. Jan. 1990. Localization of vasa, a component of Drosophila polar granules, in maternal-effect mutants that alter embryonic anteroposterior polarity. Development. 109:425-33.

Hime, G.R., J.A. Brill, and M.T. Fuller. 1996. Assembly of ring canals in the male germ line from structural components of the contractile ring. J Cell Sci. 109 (Pt 12):2779-88.

Holleran, E.A., L.A. Ligon, M. Tokito, M.C. Stankewich, J.S. Morrow, and E.L. Holzbaur. 2001. beta III spectrin binds to the Arpl subunit of dynactin. J Biol Chem. 276:36598605.

Hortsch, M., N.H. Patel, A.J. Bieber, Z.R. Traquina, and C.S. Goodman. 1990. Drosophila Neurotactin, a surface glycoprotein with homology to serine esterases, is dynamically expressed during embryogenesis. Development. 110:1327-40.

Hou, X.S., M.B. Melnick, and N. Perrimon. 1996. Marelle acts downstream of the Drosophila HOP/JAK kinase and encodes a protein similar to the mammalian STATs. Cell. 84:41 1-9. 
Hou, S.X., Z. Zheng, X. Chen, and N. Perrimon. 2002. The Jak/STAT pathway in model organisms: emerging roles in cell movement. Dev Cell. 3:765-78.

Hulskamp, M., C. Schroder, C. Pfeifle, H. Jäckle, and D. Tautz. 1989. Posterior segmentation of the Drosophila embryo in the absence of a maternal posterior organizer gene. Nature. 338:629-32.

Hunter, C., P. Sung, E.D. Schejter, and E. Wieschaus. 2002. Conserved domains of the Nullo protein required for cell-surface localization and formation of adherens junctions. Mol Biol Cell. 13:146-57.

Hunter, C., and E. Wieschaus. 2000. Regulated expression of nullo is required for the formation of distinct apical and basal adherens junctions in the Drosophila blastoderm. $J$ Cell Biol. 150:391-401.

Huynh, J.R., J.M. Shulman, R. Benton, and D. St Johnston. 2001. PAR-1 is required for the maintenance of oocyte fate in Drosophila. Development. 128:1201-9.

Huynh, J.R., and D. St Johnston. 2004. The origin of asymmetry: early polarisation of the Drosophila germline cyst and oocyte. Curr Biol. 14:R438-49.

Ibnsouda, S., F. Schweisguth, G. de Billy, and A. Vincent. 1993. Relationship between expression of serendipity alpha and cellularisation of the Drosophila embryo as revealed by interspecific transformation. Development. 119:471-83.

Imai, H., A. Narita, T.A. Schroer, and Y. Maeda. 2006. Two-dimensional averaged images of the dynactin complex revealed by single particle analysis. $J$ Mol Biol. 359:833-9.

Irish, V., R. Lehmann, and M. Akam. 1989. The Drosophila posterior-group gene nanos functions by repressing hunchback activity. Nature. 338:646-8.

Jäckle, H., and R. Jahn. 1998. Vesicle transport: klarsicht clears up the matter. Curr Biol. 8:R542-4.

Jaglarz, M.K., and K.R. Howard. 1994. Primordial germ cell migration in Drosophila melanogaster is controlled by somatic tissue. Development. 120:83-9.

Jaglarz, M.K., and K.R. Howard. 1995. The active migration of Drosophila primordial germ cells. Development. 121:3495-503.

Januschke, J., L. Gervais, S. Dass, J.A. Kaltschmidt, H. Lopez-Schier, D. St Johnston, A.H. Brand, S. Roth, and A. Guichet. 2002. Polar transport in the Drosophila oocyte requires Dynein and Kinesin I cooperation. Curr Biol. 12:1971-81.

Jenkins, A.B., J.M. McCaffery, and M. Van Doren. 2003. Drosophila E-cadherin is essential for proper germ cell-soma interaction during gonad morphogenesis. Development. 130:4417-26.

Jin, Z., and T. Xie. 2006. Germline specification: small things have a big role. Curr Biol. 16:R966-7.

Johnson, K., and A. Wodarz. 2003. A genetic hierarchy controlling cell polarity. Nat Cell Biol. 5:12-4.

Jürgens, G., E. Wieschaus, C. Nüsslein-Volhard, and H. Kluding. 1984. Mutations affecting the pattern of the larval cuticle in Drosophila melanogaster. Roux Arch. dev. 
Karch, F., W. Bender, and B. Weiffenbach. 1990. abdA expression in Drosophila embryos. Genes Dev. 4:1573-87.

Karess, R. 2005. Rod-Zw10-Zwilch: a key player in the spindle checkpoint. Trends Cell Biol. 15:386-92.

Karess, R.E., X.J. Chang, K.A. Edwards, S. Kulkarni, I. Aguilera, and D.P. Kiehart. 1991. The regulatory light chain of nonmuscle myosin is encoded by spaghetti-squash, a gene required for cytokinesis in Drosophila. Cell. 65:1177-89.

Karki, S., and E.L. Holzbaur. 1999. Cytoplasmic dynein and dynactin in cell division and intracellular transport. Curr Opin Cell Biol. 11:45-53.

Kim, H., S.C. Ling, G.C. Rogers, C. Kural, P.R. Selvin, S.L. Rogers, and V.I. Gelfand. 2007. Microtubule binding by dynactin is required for microtubule organization but not cargo transport. J Cell Biol. 176:641-51.

King, S.J., C.L. Brown, K.C. Maier, N.J. Quintyne, and T.A. Schroer. 2003. Analysis of the dynein-dynactin interaction in vitro and in vivo. Mol Biol Cell. 14:5089-97.

Klemenz, R., U. Weber, and W.J. Gehring. 1987. The white gene as a marker in a new P-element vector for gene transfer in Drosophila. Nucleic Acids Res. 15:3947-59.

Knoblich, J.A. 2000. Epithelial polarity: the ins and outs of the fly epidermis. Curr Biol. 10:R791-4.

Knust, E., and M. Leptin. 1996. Adherens junctions in the Drosophila embryo: the role of E-cadherin in their establishment and morphogenetic function. Bioessays. 18:60912.

Koch, E.A., and R.H. Spitzer. 1983. Multiple effects of colchicine on oogenesis in Drosophila: induced sterility and switch of potential oocyte to nurse-cell developmental pathway. Cell Tissue Res. 228:21-32.

Kunwar, P.S., D.E. Siekhaus, and R. Lehmann. 2006. In vivo migration: a germ cell perspective. Annu Rev Cell Dev Biol. 22:237-65.

Kunwar, P.S., M. Starz-Gaiano, R.J. Bainton, U. Heberlein, and R. Lehmann. 2003. Tre1, a $G$ protein-coupled receptor, directs transepithelial migration of Drosophila germ cells. PLoS Biol. 1 :E80.

Kusch, T., and R. Reuter. 1999. Functions for Drosophila brachyenteron and forkhead in mesoderm specification and cell signaling. Development. 126:3991-4003.

Lecuit, T. 2004. Junctions and vesicular trafficking during Drosophila cellularization. $J$ Cell Sci. 117:3427-33.

Lecuit, T., R. Samanta, and E. Wieschaus. 2002. Slam encodes a developmental regulator of polarized membrane growth during cleavage of the Drosophila embryo. Dev Cell. 2:425-36.

Lecuit, T., and E. Wieschaus. 2000. Polarized insertion of new membrane from a cytoplasmic reservoir during cleavage of the Drosophila embryo. J Cell Biol. 150:84960. 
Lehmann, R., and C. Nüsslein-Volhard. 1986. Abdominal segmentation, pole cell formation, and embryonic polarity require the localized activity of oskar, a maternal gene in Drosophila. Cell. 47:141-52.

Lehmann, R., and C. Nüsslein-Volhard. 1987. hunchback, a gene required for segmentation of an anterior and posterior region of the Drosophila embryo. Dev Biol. 119:402-17.

Lehmann, R., and C. Nüsslein-Volhard. 1991. The maternal gene nanos has a central role in posterior pattern formation of the Drosophila embryo. Development. 112:679-91.

LeMosy, E.K. 2003. Pattern formation: the eggshell holds the cue. Curr Biol. 13:R50810.

Li, W., J. Fan, M. Chen, and D.T. Woodley. 2004. Mechanisms of human skin cell motility. Histol Histopathol. 19:1311-24.

Li, J., F. Xia, and W.X. Li. 2003. Coactivation of STAT and Ras is required for germ cell proliferation and invasive migration in Drosophila. Dev Cell. 5:787-98.

Lin, H., and A.C. Spradling. 1995. Fusome asymmetry and oocyte determination in Drosophila. Dev Genet. 16:6-12.

Lin, H., L. Yue, and A.C. Spradling. 1994. The Drosophila fusome, a germline-specific organelle, contains membrane skeletal proteins and functions in cyst formation. Development. 120:947-56.

Mach, J.M., and R. Lehmann. 1997. An Egalitarian-BicaudalD complex is essential for oocyte specification and axis determination in Drosophila. Genes Dev. 1 1:423-35.

Martinez Arias, A. 1993. Development and Patterning of the Larval Epidermis of Drosophila. The Development of Drosophila melanogaster (Cold Spring Harbor Laboratory Press). 1:517-608.

Martinho, R.G., P.S. Kunwar, J. Casanova, and R. Lehmann. 2004. A noncoding RNA is required for the repression of RNApolll-dependent transcription in primordial germ cells. Curr Biol. 14:159-65.

Mazo, A.M., D.H. Huang, B.A. Mozer, and I.B. Dawid. 1990. The trithorax gene, a transacting regulator of the bithorax complex in Drosophila, encodes a protein with zincbinding domains. Proc Natl Acad Sci U S A. 87:2112-6.

McGrail, M., and T.S. Hays. 1997. The microtubule motor cytoplasmic dynein is required for spindle orientation during germline cell divisions and oocyte differentiation in Drosophila. Development. 124:2409-19.

Miller, K.G. 1995. Role of the actin cytoskeleton in early Drosophila development. Curr Top Dev Biol. 31:167-96.

Mohler, J., and E.F. Wieschaus. 1985. Bicaudal mutations of Drosophila melanogaster: alteration of blastoderm cell fate. Cold Spring Harb Symp Quant Biol. 50:105-1 1.

Mohler, J., and E.F. Wieschaus. 1986. Dominant maternal-effect mutations of Drosophila melanogaster causing the production of double-abdomen embryos. Genetics. 112:803-22.

Molitor, A. 2002. Isolierung und Charakterisierung des aeneas-Gens von Drosophila 
melanogaster.

Molyneaux, K., and C. Wylie. 2004. Primordial germ cell migration. Int J Dev Biol. 48:537-44.

Molyneaux, K.A., H. Zinszner, P.S. Kunwar, K. Schaible, J. Stebler, M.J. Sunshine, W. O'Brien, E. Raz, D. Littman, C. Wylie, and R. Lehmann. 2003. The chemokine SDF1/CXCL12 and its receptor CXCR4 regulate mouse germ cell migration and survival. Development. 130:4279-86.

Moore, L.A., H.T. Broihier, M. Van Doren, L.B. Lunsford, and R. Lehmann. 1998. Identification of genes controlling germ cell migration and embryonic gonad formation in Drosophila. Development. 125:667-78.

Moussian, B., and S. Roth. 2005. Dorsoventral axis formation in the Drosophila embryoshaping and transducing a morphogen gradient. Curr Biol. 15:R887-99.

Müller, H.A. 2000. Genetic control of epithelial cell polarity: lessons from Drosophila. Dev Dyn. 218:52-67.

Müller, H.A., and E. Wieschaus. 1996. armadillo, bazooka, and stardust are critical for early stages in formation of the zonula adherens and maintenance of the polarized blastoderm epithelium in Drosophila. J Cell Biol. 134:149-63.

Müller, P., D. Kuttenkeuler, V. Gesellchen, M.P. Zeidler, and M. Boutros. 2005. Identification of JAK/STAT signaling components by genome-wide RNA interference. Nature. 436:871-5.

Murata, Y., and R.P. Wharton. 1995. Binding of pumilio to maternal hunchback mRNA is required for posterior patterning in Drosophila embryos. Cell. 80:747-56.

Nation, J.L. 2002. Insect Physiology and Biochemistry. CRC Press. 360-364.

Navarro, C., R. Lehmann, and J. Morris. 2001. Oogenesis: Setting one sister above the rest. Curr Biol. 11:R162-5.

Navarro, C., H. Puthalakath, J.M. Adams, A. Strasser, and R. Lehmann. 2004. Egalitarian binds dynein light chain to establish oocyte polarity and maintain oocyte fate. Nat Cell Biol. 6:427-35.

Neufeld, T.P., and G.M. Rubin. 1994. The Drosophila peanut gene is required for cytokinesis and encodes a protein similar to yeast putative bud neck filament proteins. Cell. 77:371-9.

Oda, H., T. Uemura, Y. Harada, Y. Iwai, and M. Takeichi. 1994. A Drosophila homolog of cadherin associated with armadillo and essential for embryonic cell-cell adhesion. Dev Biol. 165:716-26.

Papoulas, O., T.S. Hays, and J.C. Sisson. 2005. The golgin Lava lamp mediates dyneinbased Golgi movements during Drosophila cellularization. Nat Cell Biol. 7:612-8.

Pearson, J., and A. Gonzalez-Reyes. 2004. Egalitarian and the case of the missing link. Nat Cell Biol. 6:381-3.

Peifer, M., and E. Wieschaus. 1990. The segment polarity gene armadillo encodes a functionally modular protein that is the Drosophila homolog of human plakoglobin. Cell. 63:1167-76. 
Pelissier, A., J.P. Chauvin, and T. Lecuit. 2003. Trafficking through Rab11 endosomes is required for cellularization during Drosophila embryogenesis. Curr Biol. 13:1848-57.

Perrimon, N. 1988. The maternal effect of lethal(1)discs-large-1: a recessive oncogene of Drosophila melanogaster. Dev Biol. 127:392-407.

Petronczki, M., and J.A. Knoblich. 2001. DmPAR-6 directs epithelial polarity and asymmetric cell division of neuroblasts in Drosophila. Nat Cell Biol. 3:43-9.

Pielage, J., T. Stork, I. Bunse, and C. Klambt. 2003. The Drosophila cell survival gene discs lost encodes a cytoplasmic Codanin-1-like protein, not a homolog of tight junction PDZ protein Patj. Dev Cell. 5:841-51.

Pokrywka, N.J., and E.C. Stephenson. 1991. Microtubules mediate the localization of bicoid RNA during Drosophila oogenesis. Development. 113:55-66.

Pokrywka, N.J., and E.C. Stephenson. 1995. Microtubules are a general component of mRNA localization systems in Drosophila oocytes. Dev Biol. 167:363-70.

Postner, M.A., and E.F. Wieschaus. 1994. The nullo protein is a component of the actin-myosin network that mediates cellularization in Drosophila melanogaster embryos. J Cell Sci. 107 (P† 7):1863-73.

Poulton, J.S., and W.M. Deng. 2006. Dystroglycan down-regulation links EGF receptor signaling and anterior-posterior polarity formation in the Drosophila oocyte. Proc Natl Acad Sci U S A. 103:12775-80.

Reddy, S., P. Jin, J. Trimarchi, P. Caruccio, R. Phillis, and R.K. Murphey. 1997. Mutant molecular motors disrupt neural circuits in Drosophila. J Neurobiol. 33:711-23.

Renault, A.D., Y.J. Sigal, A.J. Morris, and R. Lehmann. 2004. Soma-germ line competition for lipid phosphate uptake regulates germ cell migration and survival. Science. 305:1963-6.

Reuter, R. 1994. The gene serpent has homeotic properties and specifies endoderm versus ectoderm within the Drosophila gut. Development. 120:1 123-35.

Riechmann, V., and A. Ephrussi. 2001. Axis formation during Drosophila oogenesis. Curr Opin Genet Dev. 11:374-83.

Riggs, B., W. Rothwell, S. Mische, G.R. Hickson, J. Matheson, T.S. Hays, G.W. Gould, and W. Sullivan. 2003. Actin cytoskeleton remodeling during early Drosophila furrow formation requires recycling endosomal components Nuclear-fallout and Rabl1. $J$ Cell Biol. 163:143-54.

Rivera-Pomar, R., and H. Jäckle. 1996. From gradients to stripes in Drosophila embryogenesis: filling in the gaps. Trends Genet. 12:478-83.

Robertson, H.M., C.R. Preston, R.W. Phillis, D.M. Johnson-Schlitz, W.K. Benz, and W.R. Engels. 1988. A stable genomic source of $\mathrm{P}$ element transposase in Drosophila melanogaster. Genetics. 118:461-70.

Roth, S. 2003. The origin of dorsoventral polarity in Drosophila. Philos Trans $R$ Soc Lond B Biol Sci. 358:1317-29; discussion 1329. 
Rothwell, W.F., P. Fogarty, C.M. Field, and W. Sullivan. 1998. Nuclear-fallout, a Drosophila protein that cycles from the cytoplasm to the centrosomes, regulates cortical microfilament organization. Development. 125:1295-303.

Rubin, G.M., and A.C. Spradling. 1982. Genetic transformation of Drosophila with transposable element vectors. Science. 218:348-53.

Sahai, E. 2005. Mechanisms of cancer cell invasion. Curr Opin Genet Dev. 15:87-96.

Sambrook, J., E. Fritsch, and T. Maniatis. 1989. Molecular Cloning: A Laboratory Manual. Cold Spring Harbor Laboratory Press.

Santos, A.C., and R. Lehmann. 2004. Isoprenoids control germ cell migration downstream of HMGCOA reductase. Dev Cell. 6:283-93.

Sasamura, T., T. Kobayashi, S. Kojima, H. Qadota, Y. Ohya, I. Masai, and Y. Hotta. 1997. Molecular cloning and characterization of Drosophila genes encoding small GTPases of the rab and rho families. Mol Gen Genet. 254:486-94.

Sato, K., and A. Nakano. 2007. Mechanisms of COPIl vesicle formation and protein sorting. FEBS Lett.

Schafer, D.A., S.R. Gill, J.A. Cooper, J.E. Heuser, and T.A. Schroer. 1994. Ultrastructural analysis of the dynactin complex: an actin-related protein is a component of a filament that resembles F-actin. $J$ Cell Biol. 126:403-12.

Schejter, E.D., and E. Wieschaus. 1993. Bottleneck acts as a regulator of the microfilament network governing cellularization of the Drosophila embryo. Cell. 75:373-85.

Schneider, I.C., and J.M. Haugh. 2006. Mechanisms of gradient sensing and chemotaxis: conserved pathways, diverse regulation. Cell Cycle. 5:1 130-4.

Schroer, T.A. 1994. New insights into the interaction of cytoplasmic dynein with the actin-related protein, Arp 1. J Cell Biol. 127:1-4.

Schroer, T.A. 2004. Dynactin. Annu Rev Cell Dev Biol. 20:759-79.

Schroer, T.A., J.B. Bingham, and S.R. Gill. 1996. Actin-related protein 1 and cytoplasmic dynein-based motility - what's the connection? Trends Cell Biol. 6:212-5.

Schupbach, T. 1987. Germ line and soma cooperate during oogenesis to establish the dorsoventral pattern of egg shell and embryo in Drosophila melanogaster. Cell. 49:699-707.

Schweisguth, F., J.A. Lepesant, and A. Vincent. 1990. The serendipity alpha gene encodes a membrane-associated protein required for the cellularization of the Drosophila embryo. Genes Dev. 4:922-31.

Sharp, D.J., G.C. Rogers, and J.M. Scholey. 2000. Cytoplasmic dynein is required for poleward chromosome movement during mitosis in Drosophila embryos. Nat Cell Biol. 2:922-30.

Simpson, L., and E. Wieschaus. 1990. Zygotic activity of the nullo locus is required to stabilize the actin-myosin network during cellularization in Drosophila. Development. 110:851-63. 
Sisson, J.C., C. Field, R. Ventura, A. Royou, and W. Sullivan. 2000. Lava lamp, a novel peripheral golgi protein, is required for Drosophila melanogaster cellularization. $J$ Cell Biol. 151:905-18.

Skaer, H. 1993. The Alimentary Canal. The Development of Drosophila melanogaster Cold Spring Harbor Laboratory Press. 2:941-1012.

Snodgrass, R.E. 1993. Principles of insect morphology. Cornell university press.

Spradling, A.C. 1993. Developmental Genetics of Oogenesis. The Development of Drosophila melanogaster (Cold Spring Harbor Laboratory Press). 1:1-70.

St Johnston, D., and C. Nüsslein-Volhard. 1992. The origin of pattern and polarity in the Drosophila embryo. Cell. 68:201-19.

Starz-Gaiano, M., N.K. Cho, A. Forbes, and R. Lehmann. 2001. Spatially restricted activity of a Drosophila lipid phosphatase guides migrating germ cells. Development. 128:983-91.

Stein, J.A., H.T. Broihier, L.A. Moore, and R. Lehmann. 2002. Slow as molasses is required for polarized membrane growth and germ cell migration in Drosophila. Development. 129:3925-34.

Storto, P.D., and R.C. King. 1989. The role of polyfusomes in generating branched chains of cystocytes during Drosophila oogenesis. Dev Genet. 10:70-86.

Strand, D., I. Raska, and B.M. Mechler. 1994. The Drosophila lethal(2)giant larvae tumor suppressor protein is a component of the cytoskeleton. J Cell Biol. 127:1345-60.

Strickland, L.I., and D.R. Burgess. 2004. Pathways for membrane trafficking during cytokinesis. Trends Cell Biol. 14:115-8.

Strome, S., and R. Lehmann. 2007. Germ versus soma decisions: lessons from flies and worms. Science. 316:392-3.

Struhl, G. 1989. Morphogen gradients and the control of body pattern in insect embryos. Ciba Found Symp. 144:65-86; discussion 86-91, 92-8.

Struhl, G., K. Struhl, and P.M. Macdonald. 1989. The gradient morphogen Bicoid is a concentration-dependent transcriptional activator. Cell. 57:1259-73.

Tabata, T., and Y. Takei. 2004. Morphogens, their identification and regulation. Development. 131:703-12.

Tautz, D. 1988. Regulation of the Drosophila segmentation gene hunchback by two maternal morphogenetic centres. Nature. 332:281-4.

Tepass, U. 1996. Crumbs, a component of the apical membrane, is required for zonula adherens formation in primary epithelia of Drosophila. Dev Biol. 177:217-25.

Tepass, U., E. Gruszynski-DeFeo, T.A. Haag, L. Omatyar, T. Torok, and V. Hartenstein. 1996. shotgun encodes Drosophila E-cadherin and is preferentially required during cell rearrangement in the neurectoderm and other morphogenetically active epithelia. Genes Dev. 10:672-85.

Tepass, U., G. Tanentzapf, R. Ward, and R. Fehon. 2001. Epithelial cell polarity and cell junctions in Drosophila. Annu Rev Genet. 35:747-84. 
Tepass, U., C. Theres, and E. Knust. 1990. crumbs encodes an EGF-like protein expressed on apical membranes of Drosophila epithelial cells and required for organization of epithelia. Cell. 61:787-99.

Theurkauf, W.E. 1994. Actin cytoskeleton. Through the bottleneck. Curr Biol. 4:76-8.

Theurkauf, W.E., B.M. Alberts, Y.N. Jan, and T.A. Jongens. 1993. A central role for microtubules in the differentiation of Drosophila oocytes. Development. 118:1 169-80.

Theurkauf, W.E., S. Smiley, M.L. Wong, and B.M. Alberts. 1992. Reorganization of the cytoskeleton during Drosophila oogenesis: implications for axis specification and intercellular transport. Development. 115:923-36.

Tomancak, P., F. Piano, V. Riechmann, K.C. Gunsalus, K.J. Kemphues, and A. Ephrussi. 2000. A Drosophila melanogaster homologue of Caenorhabditis elegans par-1 acts at an early step in embryonic-axis formation. Nat Cell Biol. 2:458-60.

Turner, F.R., and A.P. Mahowald. 1976. Scanning electron microscopy of Drosophila embryogenesis. 1. The structure of the egg envelopes and the formation of the cellular blastoderm. Dev Biol. 50:95-108.

Ullrich, O., S. Reinsch, S. Urbe, M. Zerial, and R.G. Parton. 1996. Rab11 regulates recycling through the pericentriolar recycling endosome. J Cell Biol. 135:913-24.

Van Buskirk, C., and T. Schupbach. 1999. Versatility in signaling: multiple responses to EGF receptor activation during Drosophila oogenesis. Trends Cell Biol. 9:1-4.

Van Doren, M., H.T. Broihier, L.A. Moore, and R. Lehmann. 1998. HMG-CoA reductase guides migrating primordial germ cells. Nature. 396:466-9.

Van Doren, M., W.R. Mathews, M. Samuels, L.A. Moore, H.T. Broihier, and R. Lehmann. 2003. fear of intimacy encodes a novel transmembrane protein required for gonad morphogenesis in Drosophila. Development. 130:2355-64.

Vincent, A., H.V. Colot, and M. Rosbash. 1985. Sequence and structure of the serendipity locus of Drosophila melanogaster. A densely transcribed region including a blastoderm-specific gene. J Mol Biol. 186:149-66.

Wakimoto, B.T., F.R. Turner, and T.C. Kaufman. 1984. Defects in embryogenesis in mutants associated with the antennapedia gene complex of Drosophila melanogaster. Dev Biol. 102:147-72.

Wang, C., and R. Lehmann. 1991. Nanos is the localized posterior determinant in Drosophila. Cell. 66:637-47.

Warn, R.M. 1986. The cytoskeleton of the early Drosophila embryo. J Cell Sci Suppl. 5:311-28.

Warn, R.M., B. Bullard, and R. Magrath. 1980. Changes in the distribution of cortical myosin during the cellularization of the Drosophila embryo. J Embryol Exp Morphol. 57:167-76.

Warn, R.M., L. Flegg, and A. Warn. 1987. An investigation of microtubule organization and functions in living Drosophila embryos by injection of a fluorescently labeled antibody against tyrosinated alpha-tubulin. J Cell Biol. 105:1721-30. 
Warrior, R. 1994. Primordial germ cell migration and the assembly of the Drosophila embryonic gonad. Dev Biol. 166:180-94.

Watson, P., R. Forster, K.J. Palmer, R. Pepperkok, and D.J. Stephens. 2005. Coupling of ER exit to microtubules through direct interaction of COPII with dynactin. Nat Cell Biol. 7:48-55.

Weil, T.T., K.M. Forrest, and E.R. Gavis. 2006. Localization of bicoid mRNA in late oocytes is maintained by continual active transport. Dev Cell. 11:251-62.

Welte, M.A. 2004. Bidirectional transport along microtubules. Curr Biol. 14:R525-37.

Welte, M.A., S.P. Gross, M. Postner, S.M. Block, and E.F. Wieschaus. 1998. Developmental regulation of vesicle transport in Drosophila embryos: forces and kinetics. Cell. 92:547-57.

White, R.A., and R. Lehmann. 1986. A gap gene, hunchback, regulates the spatial expression of Ultrabithorax. Cell. 47:31 1-21.

Whited, J.L., A. Cassell, M. Brouillette, and P.A. Garrity. 2004. Dynactin is required to maintain nuclear position within postmitotic Drosophila photoreceptor neurons. Development. 131:4677-86.

Wieschaus, E., C. Nüsslein-Volhard, and H. Kluding. 1984. Kruppel, a gene whose activity is required early in the zygotic genome for normal embryonic segmentation. Dev Biol. 104:172-86.

Williams, P.L., L. H. Bannister, M. M. Berry, P. Collins, M. Dyson, J. E. Dussek, M. W. J. Fergusson. 1995. Gray's Anatomy. Churchhill Livingstone. 67-74.

Williamson, A., and R. Lehmann. 1996. Germ cell development in Drosophila. Annu Rev Cell Dev Biol. 12:365-91.

Wodarz, A., F. Grawe, and E. Knust. 1993. CRUMBS is involved in the control of apical protein targeting during Drosophila epithelial development. Mech Dev. 44:175-87.

Wodarz, A., U. Hinz, M. Engelbert, and E. Knust. 1995. Expression of crumbs confers apical character on plasma membrane domains of ectodermal epithelia of Drosophila. Cell. 82:67-76.

Wodarz, A., A. Ramrath, A. Grimm, and E. Knust. 2000. Drosophila atypical protein kinase $\mathrm{C}$ associates with Bazooka and controls polarity of epithelia and neuroblasts. $J$ Cell Biol. 150:1361-74.

Woese, C.R., O. Kandler, and M.L. Wheelis. 1990. Towards a natural system of organisms: proposal for the domains Archaea, Bacteria, and Eucarya. Proc Natl Acad Sci U S A. 87:4576-9.

Young, P.E., A.M. Richman, A.S. Ketchum, and D.P. Kiehart. 1993. Morphogenesis in Drosophila requires nonmuscle myosin heavy chain function. Genes Dev. 7:29-41.

Zen, K., and C.A. Parkos. 2003. Leukocyte-epithelial interactions. Curr Opin Cell Biol. 15:557-64.

Zhang, N., J. Zhang, Y. Cheng, and K. Howard. 1996. Identification and genetic analysis of wunen, a gene guiding Drosophila melanogaster germ cell migration. Genetics. 143:1231-41. 


\section{Acknowledgements}

Die vorliegende Doktorarbeit wurde am Max-Planck-Institut für biophysikalische Chemie, Göttingen, in der Abteilung für Molekulare Entwicklungsbiologie, geleitet von Prof. Dr Dr h.c. Herbert Jäckle, unter Anleitung von Dr Gerd Vorbrüggen erstellt.

Mein erster Dank gilt Dr Gerd Vorbrüggen für die Betreung meiner wissenschaftlichen Arbeit, für seine beständige Hilfs- und Diskussionsbereitschaft und die Bereitstellung dieses Projekts.

Besonders danke ich auch Prof. Dr Dr h.c. Herbert Jäckle für seine intellektuelle Unterstützung und Finanzierung dieser Arbeit.

Ihm und den weiteren Mitgliedern meines Promotionsausschusses, Prof. Dr Reinhard Jahn und Prof. Dr Mary Osborn danke ich für die wissenschaftliche Begleitung meiner Doktorarbeit und fruchtbare Dikussionen.

Den Mitgliedern des "International MSc/PhD Program Molecular Biology", insbesondere Dr Steffen Burckhardt danke ich für ihr Engagement und für den vertravensvollen und unbürokratischen Umgang.

Weiterhin gilt mein Dank Dr Ralf Pflanz und Dr Alf Herzig für ihre stetige Bereitschaft, mir bei experimentellen und technischen Problemen mit Rat und Tat zur Seite zu stehen. Darüber hinaus danke ich Dr. Ralf Pflanz für die kritische Revision dieses Manuskripts.

Iris Plischke danke ich für Embryo-Injektionen, Gordon Dowe für DNASequenzierungen

Bhavna Chanana, Dr Alexey Matyash und Tatyana Koledachkina und den anderen Mitgliedern der Abteilung danke ich für die großartige Arbeitsatmosphäre und Diskussionen über die Wissenschaft, das Leben und Tom Wolfe.

Dr Andreas Molitor danke ich für geleistete Vorarbeiten, Dr Jochen Deckert und Kalpana Singh für ihre Beteiligung an der Arbeit im Zuge ihrer Laborrotationen.

Mein herzlichster Dank gilt meiner Frau Mareike für ihre Liebe und Unterstützung.

Ich danke meinem Vater, dessen Zielstrebigkeit und Arbeitsethos aber auch Humor für mich stete Motivation und Inspiration sind.

Meiner Mutter danke Ich für ihre Führsorglichkeit und ihr immer offenes Ohr.

Meiner Tochter Pia, meinem gelungensten entwicklungsbiologischen Experiment, danke ich für ihr herzliches Lachen und ihr unstillbares Interesse an Mama, Papa, Autos, Nasen und Lampen. 


\section{Curriculum Vitae}

Roland Graf

Born in Hannover, 14.11.1977

Scientific education

2004-present

Dissertation at the Max-Planck Institute for biophysical Chemistry, Göttingen, in the department for Molecular Developmental Biology, headed by Prof. Dr Dr h.c. Herbert Jäckle, in the group of Dr. Gerd Vorbrüggen

2004

Graduation with a MSC degree from the International MSc/PhD Program Molecular Biology / International MaxPlanck Research School, Göttingen

$2002-2004$

Studies in Molecular Biology at International MSc/PhD Program Molecular Biology / International Max-Planck Research School, Göttingen

Studies in Biology at the Georg-August-University, Göttingen

1998-1999

Studies in Chemistry at the Georg-August-University, Göttingen 


\section{Publications}

Chanana B1, Graf R1, Koledachkina T, Pflanz R, Vorbrüggen G. 2007. alpha(PS2) integrin-mediated muscle attachment in Drosophila requires the ECM protein Thrombospondin. Mech Dev. Jul;124(6):463-75.

'both authors contributed equally to this work

Bayrhuber M1, Graf R1, Ferber M, Zweckstetter M, Imperial J, Garrett JE, Olivera BM, Terlau H, Becker S. 2006. Production of recombinant Conkunitzin-S1 in Escherichia coli Protein Expr Purif. Jun;47(2):640-4.

'both authors contributed equally to this work

Bayrhuber M, Vijayan V, Ferber M, Graf R, Korukottu J, Imperial J, Garrett JE, Olivera $B M$, Terlau H, Zweckstetter M, Becker S. 2005. Conkunitzin-S1 is the first member of a new Kunitz-type neurotoxin family - structural and functional characterization. J Biol Chem. Jun 24;280(25):23766-70. 